\title{
RESOLVE AND ECO: THE HALO MASS-DEPENDENT SHAPE OF GALAXY STELLAR AND BARYONIC MASS FUNCTIONS
}

\author{
Kathleen D. Eckert ${ }^{1}$, Sheila J. Kannappan ${ }^{1}$, David V. Stark ${ }^{1,2}$, Amanda J. Moffett ${ }^{1,3}$, Andreas A. Berlind ${ }^{3,4}$, and \\ MARK A. NORRIS ${ }^{1,5}$ \\ ${ }^{1}$ Department of Physics and Astronomy, University of North Carolina, 141 Chapman Hall CB 3255, Chapel Hill, NC 27599, USA; keckert@physics.unc.edu \\ ${ }^{2}$ Kavli Institute for the Physics and Mathematics of the Universe (IPMU), The University of Tokyo, 5-1-5 Kashiwanoha, Kashiwa, 277-8583, Japan \\ ${ }^{3}$ International Centre for Radio Astronomy Research (ICRAR), The University of Western Australia, 35 Stirling Highway, Crawley, WA 6009, Australia \\ ${ }^{4}$ Department of Physics and Astronomy, Vanderbilt University, PMB 401807, 2401 Vanderbilt Place, Nashville, TN 37240-1807, USA \\ 5 Jeremiah Horrocks Institute, University of Central Lancashire, Preston, PR1 2HE, UK \\ Received 2015 October 16; accepted 2016 April 13; published 2016 June 21
}

\begin{abstract}
In this work, we present galaxy stellar and baryonic (stars plus cold gas) mass functions (SMF and BMF) and their halo mass dependence for two volume-limited data sets. The first, RESOLVE-B, coincides with the Stripe 82 footprint and is extremely complete down to baryonic mass $M_{\text {bary }} \sim 10^{9.1} M_{\odot}$, probing the gas-rich dwarf regime below $M_{\text {bary }} \sim 10^{10} M_{\odot}$. The second, ECO, covers a $\sim 40 \times$ larger volume (containing RESOLVE-A) and is complete to $M_{\text {bary }} \sim 10^{9.4} M_{\odot}$. To construct the SMF and BMF we implement a new "cross-bin sampling" technique with Monte Carlo sampling from the full likelihood distributions of stellar or baryonic mass. Our SMFs exhibit the "plateau" feature starting below $M_{\text {star }} \sim 10^{10} M_{\odot}$ that has been described in prior work. However, the BMF fills in this feature and rises as a straight power law below $\sim 10^{10} M_{\odot}$, as gas-dominated galaxies become the majority of the population. Nonetheless, the low-mass slope of the BMF is not as steep as that of the theoretical dark matter halo MF. Moreover, we assign group halo masses by abundance matching, finding that the SMF and BMF, separated into four physically motivated halo mass regimes, reveal complex structure underlying the simple shape of the overall MFs. In particular, the satellite MFs are depressed below the central galaxy MF "humps" in groups with mass $<10^{13.5} M_{\odot}$ yet rise steeply in clusters. Our results suggest that satellite destruction and stripping are active from the point of nascent group formation. We show that the key role of groups in shaping MFs enables reconstruction of a given survey's SMF or BMF based on its group halo mass distribution.
\end{abstract}

Key words: galaxies: luminosity function, mass function - methods: statistical - surveys

Supporting material: machine-readable table

\section{INTRODUCTION}

Galaxy luminosity and mass functions are key tools for understanding the distribution of matter in the universe. The shape of the luminosity function (LF) reveals the mass assembly of galaxies through hierarchical evolution, as the dwarf galaxies that dominate the galaxy population eventually merge to form the rarer bright galaxy population. As galaxies merge to form larger structures, their host halos also merge and grow, implying a relationship between the LF and halo mass function (HMF). Despite this link between galaxies and their host halos, the faintend slope for the LF has been measured to be much shallower than the low-mass slope for the HMF. While the low-mass slope of the HMF is often reported to be $\alpha_{\mathrm{HMF}}=-2$ (e.g., Press \& Schechter 1974 and Springel et al. 2005), for the Sloan Digital Sky Survey (SDSS, York et al. 2000), Blanton et al. (2003a) measure the faint end of the LF to be $\alpha_{\mathrm{LF}}=-1.05$.

The faint-end slope of the LF, however, is dependent on environment such that the faint-end slope in clusters is much steeper than in less dense environments (Tully et al. 2002). In fact, Popesso et al. (2006) measured faint-end slopes $\alpha_{\mathrm{LF}} \sim-2$ in a large sample of clusters. Other previous studies of the cluster LF, however, have found varying faint-end slopes from -1.0 to -1.4 (Dressler 1978; Valotto et al. 1997; Goto et al. 2002). One proposed explanation for the discrepancy between low galaxy number counts in the field versus in clusters is that galaxies in more dense environments formed earlier and faster, before the reionization of hydrogen by the UV background, whereas galaxies in less dense environments took longer to form. The latter galaxies were therefore "squelched" by reionization, which heated the gas so that the galaxy formation was delayed until the reionized gas could recool and form stars (Thoul \& Weinberg 1996; Gnedin \& Ostriker 1997; Becker et al. 2001; Pritchard et al. 2010).

While the LF offers clues to the mass assembly of galaxies, it is also sensitive to star-formation histories. With the development of multiband photometric surveys, we can now estimate stellar masses for galaxies with population synthesis modeling and study the buildup of stellar mass through the stellar mass function (SMF). Working at $z<0.05$ and $z=0.2-1$, respectively, Baldry et al. (2008) and Drory et al. (2009) find that the traditional Schechter function does not adequately describe the SMF, which exhibits a "dip" or "plateau" at stellar masses $\sim 10^{10} M_{\odot}$ before rising more steeply for lower mass galaxies. Instead the authors use a double Schechter function to fit the SMF's more complex structure.

These discrepancies in mass function shape motivate the desire to study galaxy mass functions less sensitive to galaxy star-formation properties. The baryonic mass function (BMF), or frequency distribution of galaxies in stellar plus cold gas mass, is one step closer to a total mass function than the SMF. In this work we define baryonic mass as stars plus cold atomic gas (neglecting the cold molecular as well as warm and hot gas components). In general, cold atomic gas dominates the cold gas mass in galaxies, except in large spirals, for which the total cold gas content is usually less than the stellar mass (e.g., Casoli et al. 1998; Kannappan et al. 2013; Boselli et al. 2014). 
At high masses, we expect the BMF and SMF to be the same. Above stellar masses of $\sim 10^{10.5} M_{\odot}$, the bimodality mass scale (Kauffmann et al. 2003b), galaxies are increasingly spheroiddominated with old stellar populations and little to no recent star formation. Since these galaxies have minimal cold gas reservoirs, their baryonic masses are roughly equal to their stellar masses. Below the bimodality mass scale, gas becomes increasingly important, so we expect to see a divergence between the BMF and SMF around or below this scale. The divergence may be expected to become more extreme below the gas-richness threshold mass identified by Kannappan et al. (2013), hereafter K13, as $M_{\text {star }} \sim 10^{9.7} M_{\odot}$ or $M_{\text {bary }} \sim 10^{9.9} M_{\odot}$, below which gasdominated galaxies become the norm (see also Kannappan 2004; Kannappan \& Wei 2008; Kannappan et al. 2009). The gas mass in such gas-dominated galaxies shifts them to more massive bins by $\gtrsim 0.3$ dex, so we want to investigate whether these galaxies fill in the dip feature seen in the SMF.

Early work by Bell et al. (2003a) investigated the SMF and BMF, showing a divergence between the two at low masses. This work, however, did not reveal or investigate any structure beyond a single Schechter function form, perhaps due to systematics in the stellar mass estimation (Kannappan \& Gawiser 2007; Roediger \& Courteau 2015) or gas mass estimation methodology. Recent studies of the SMF and BMF find a dip only in the SMF, which suggests the dip is purely due to neglect of the cold gas mass (Baldry et al. 2008; Papastergis et al. 2012). This result implies that the BMF is a more fundamental way to characterize galaxy populations than the $\mathrm{SMF}$ in that it better reflects the total galaxy mass.

Thus, to relate the mass assembly of galaxies and halos, we would like to examine the BMF and in particular how the BMF depends on environment. It has been predicted that the BMF might be invariant across environments, as opposed to the faint-end slope of the LF, which is observed to steepen in cluster versus field environments (Bell et al. 2003a). This idea is based on the assumption of gas recooling after reionization: galaxies in high-density environments form earlier and faster, using up their gas to form stars before reionization hits, so the majority of cold baryons in the cluster environment are associated with stars, while galaxies in low-mass environments form later and are initially "squelched" by reionization, eventually recovering their baryonic mass as the gas recools over cosmic time (Bullock et al. 2000; Somerville 2002). Thus the BMF (stars plus cold gas) would now be constant across environments. While there has been no measurement of an environment-dependent low-mass slope for the BMF, estimates for the low-mass slope of the overall $\mathrm{BMF}$ range from $\alpha_{\mathrm{BMF}}=-1.2$ (Bell et al. 2003a; Papastergis et al. 2012) to $\alpha_{\mathrm{BMF}}=-1.8$ (Baldry et al. 2008), and the source of these variations is unclear.

One possible reason for the differences between measurements of low-mass slope could be that the methods used in each study have varied widely. A steep low-mass slope is found in Baldry et al. (2008), where baryonic mass is inferred using the stellar mass-metallicity relation combined with the relation between metallicity and stellar mass fraction, i.e., the fraction of baryons locked up in stars, to obtain total baryonic masses that implicitly include all gas, cold and warm. Both Bell et al. (2003a) and Read \& Trentham (2005) employ indirect methods of estimating the cold atomic and molecular gas based on photometric properties, respectively the $K$-band luminosity$r_{e}$ plane and galaxy morphology, both of which have large scatter. The BMF from Papastergis et al. (2012) is constructed from HI measurements from the blind wide-area $21 \mathrm{~cm}$ ALFALFA survey (Haynes et al. 2011) and does not include molecular gas (similar to this work). Since ALFALFA is fluxlimited, however, gas-rich but low-mass galaxies will be detected only nearby, requiring large statistical corrections at the faint end of the mass function.

A second possible reason for the differences between studies could be that the environment actually does affect the BMF, via physics additional to recooling since reionization. In high-mass halos, cold or warm gas may be stripped as galaxies enter the group environment, effectively moving the galaxies to lower baryonic mass, or causing them to lose their future supply of cooling (sub)halo gas. In low-mass group halos, cosmic accretion of gas onto the halo as well as halo gas recooling may increase the gas content of galaxies and thus renew the pool of gas as stars are formed. From these two examples, it is evident that environment can play a multifaceted role in shaping the galaxy BMF. Any two given data sets may contain widely varying environment distributions due to cosmic variance, so differences in the environments sampled by previous BMF studies may contribute to inconsistent low-mass slopes.

In this work we present the SMF and BMF for two volumelimited data sets: the REsolved Spectroscopy of a Local VolumE (RESOLVE) survey (S. J. Kannappan et al. 2016, in preparation) and the Environmental COntext (ECO) catalog (Moffett et al. 2015). Because these two data sets are volume-limited, we can examine the shape of the galaxy mass function and its dependence on halo mass (the proxy for environment used in this work) without the statistical completeness corrections required for flux-limited surveys. Both data sets are more complete than the SDSS main redshift survey, and one, the portion of the RESOLVE survey overlapping Stripe 82 (RESOLVE-B), offers unprecedented completeness that enables calculation of empirical completeness corrections for the other, the ECO catalog containing RESOLVE-A. Our use of volume-limited data sets enables robust group identification so that we can quantify group halo mass and directly examine its effect on the mass function shape. To obtain unbiased gas data for gas-rich yet low- $M_{\mathrm{HI}}$ galaxies, we require $\mathrm{HI}$ data that are fractional-mass limited, i.e., adaptively sensitive to a limiting atomic gas mass of $1.4 M_{\mathrm{HI}}<0.05 M_{\text {star }}$. Thus for atomic gas measurements, we use a combination of the highly complete fractional-mass-limited RESOLVE HI census from Stark et al. (2016), additional archival HI data for ECO, and empirically estimated "photometric gas fractions" (PGF) using the probability density field approach of Eckert et al. (2015), hereafter E15. Our stellar mass estimation applies spectral energy distribution (SED) fitting to custom reprocessed NUVugrizYJHK photometry, optimized for recovery of extended light (E15). Finally, we take into account the full likelihood distributions of both stellar and gas masses when computing the SMF and BMF via a new cross-bin sampling approach.

As anticipated, we find that the BMF diverges significantly from the SMF below the gas-richness threshold scale $M_{\text {bary }} \sim 10^{9.9} M_{\odot}$ and rises as a straight power law, filling in where the SMF dips. However, we find that the overall BMF hides significant substructure as a function of group halo mass. We break down our mass functions into four physically motivated halo mass regimes, finding that although mass functions of central galaxies are discrete "humps" increasing in mass as halo mass increases, satellite galaxy mass functions show much greater complexity. These shapes suggest a connection between group 
formation and satellite destruction from the point of first group formation. As evidence that the primary environmental processes affecting the BMF occur on group scales, we show that it is possible to combine the mass functions broken down by group halo mass regime for ECO with the different frequency distribution of group mass halos for RESOLVE-B to produce the observed mass function of RESOLVE-B.

This work is laid out as follows. In Section 2 we describe the surveys used for this work. In Section 3 we describe the data, including photometric reprocessing, stellar mass estimation, HI mass measurement, baryonic masses, and halo mass determination. We conclude Section 3 with a discussion of completeness corrections and mass completeness limits. In Section 4 we describe our new cross-bin sampling technique to measure the SMF and BMF. In Section 5 we present the SMF and BMF and break them down by halo mass and central or satellite designation. In Section 6 we discuss the role of the group halo mass environment in shaping the galaxy population and the connection between the galaxy BMF and the theoretical HMF. Finally, in Section 7 we summarize our conclusions.

For distance measurements and other derived quantities in this work, we assume a standard $\Lambda$ CDM cosmology with $\Omega_{m}=0.3, \Omega_{\Lambda}=0.7$, and $H_{o}=70 \mathrm{~km} \mathrm{~s}^{-1} \mathrm{Mpc}^{-1}$.

\section{DATA SETS}

To measure the SMF and BMF, we use two volume-limited data sets, the B-semester subvolume of the RESOLVE survey, RESOLVE-B (Kannappan \& Wei 2008; Kannappan et al. 2016, in preparation), and the ECO catalog (Moffett et al. 2015, hereafter M15), which contains the RESOLVE-A subvolume. RESOLVE is a volume and roughly baryonic mass limited survey of $\sim 52,100 \mathrm{Mpc}^{3}$ of the $z \sim 0$ universe. It is smaller but more complete than ECO and is acquiring new $21 \mathrm{~cm}$ and optical spectroscopy to conduct a full mass census of stars, gas, and dark matter. The RESOLVE-A $21 \mathrm{~cm}$ census was used in E15 to calibrate gas mass estimators based on the photometric properties of galaxies. The ECO catalog is $~ 10$ times larger than RESOLVE, providing better statistics but reduced completeness, and is based on archival data except in its overlap with RESOLVE-A.

\subsection{Common Features of RESOLVE and ECO}

Both data sets are based on the SDSS main redshift survey, but include additional redshifts from various archival sources: the Updated Zwicky Catalog (Falco et al. 1999), HyperLEDA (Paturel et al. 2003), 6dF (Jones et al. 2009), 2dF (Colless et al. 2001), GAMA (Driver et al. 2011), ALFALFA (Haynes et al. 2011), and new RESOLVE observations (S. J. Kannappan et al. 2016, in preparation). RESOLVE-B benefits from extra redshifts taken during repeat SDSS observations of the Stripe 82 footprint. All of these additional redshift sources help to recover both large and small galaxies originally missed by the main SDSS survey for various reasons, including fiber collisions, which affect galaxies of all brightnesses, as well as "shredding" by the SDSS photometric pipeline, which primarily affects galaxies with low surface brightness (Blanton et al. 2005).

For both RESOLVE and ECO we have custom reprocessed near-UV, optical, and near-IR photometry, as described in E15 and M15. We have computed stellar masses using the SED fitting routine described in K13. The directly measured HI census is far more complete for RESOLVE than for ECO, but PGFs and upper limits have been computed in the same way for both data sets. We have also performed group finding using a modified version of the Berlind et al. (2006) friends-of-friends (FOF) algorithm to assign groups and performed halo abundance matching to assign halo masses. The halo masses in this work are offset $\sim 0.15$ dex toward higher masses than those reported in M15 (see Section 3.5.2). When performing group finding, we use expanded RESOLVE and ECO data sets that provide a buffer region in $\mathrm{cz}$ space to recover galaxies in groups and clusters with large peculiar velocities. For RESOLVE-B this buffer region is $\pm 250 \mathrm{~km} \mathrm{~s}^{-1}$ on either side of the volume. For ECO, which contains several large groups and clusters (i.e., with large peculiar velocities) near the survey redshift boundaries, the buffer region extends $\pm 470 \mathrm{~km} \mathrm{~s}^{-1}$ on either side of the volume. RESOLVE's largest cluster is fortuitously centered in the survey redshift range. To determine final membership within RESOLVE-B and ECO, we require that the group rather than the galaxy redshift belongs within the defined volume (for $N=1$ groups, the group redshift is the galaxy redshift). In the following sections we present a more detailed description of both data sets and their relative strengths and weaknesses for the purposes of this paper.

\subsection{RESOLVE-B}

RESOLVE-B is a subset of the RESOLVE survey located within the SDSS Stripe 82 footprint and encompasses a volume of $\sim 13,700 \mathrm{Mpc}^{3}$, with coordinate and redshift ranges of $22 \mathrm{~h}<$ R.A. $<3 \mathrm{~h},-1.25<$ decl. $<+1.25$, and $4500 \mathrm{~km} \mathrm{~s}^{-1}<\mathrm{c} z<7000 \mathrm{~km} \mathrm{~s}^{-1}$ (see Figure 1(a)).

RESOLVE-B is a powerful data set for measuring the galaxy SMF and BMF due to its exceptional completeness above and beyond the main SDSS redshift survey, allowing us to analyze mass functions well into the gas-rich dwarf regime without statistical completeness corrections. In Figure 1(b), the luminosity distribution of galaxies from the main SDSS redshift survey with $M_{r \text {,petro }}<-17.23$ (corresponding to $m_{r \text {,petro }}=17.77$ at the far side of the RESOLVE volume) "RESOLVE-B,orig" is shown in green. Note that the figure is plotted using our own $M_{r \text {, tot }}$ values (see Section 3.1 and E15), which are typically $\sim 0.1$ mag brighter than $M_{r \text {,petro }}$ (all magnitudes include foreground extinction corrections), so the RESOLVE-B,orig luminosity completeness limit occurs at $M_{r \text {,tot }}=-17.33 \mathrm{mag}$. RESOLVE-B,orig consists of 329 galaxies brighter than this limit. In contrast, the full RESOLVE-B data set is shown in black crosshatch and consists of 426 galaxies brighter than -17.33 , or $28 \%$ more galaxies than in the SDSS main redshift survey (see Section 3.6.1 for more discussion on the reasons for incompleteness). Because of the large number of extra redshifts, we adopt a RESOLVE-B sample definition of $M_{r \text {,tot }}=-17$ for a total of 487 galaxies brighter than this fainter luminosity completeness limit and meeting the group $\mathrm{cz}$ criterion. Allowing all galaxies with known redshift inside the volume and not cutting by absolute $r$-band magnitude, there are 679 galaxies inside the RESOLVE-B subvolume.

In RESOLVE-B, we take advantage of the wealth of imaging data available in the Stripe 82 legacy footprint, including deep SDSS ugriz coadds, Medium Imaging Survey (MIS) depth GALEX NUV and/or Swift UVOT imaging for $98 \%$ of RESOLVE-B galaxies, 2MASS $J H K$, and deeper UKIDSS $Y H K$. We have obtained $21 \mathrm{~cm}$ coverage from both the ALFALFA survey (covering the $0^{\circ}-1.25$ northern strip) and pointed observations with the Arecibo and Green Bank telescopes, obtaining reliable detections or strong upper limits $\left(1.4 M_{\mathrm{HI}}<0.05 M_{\mathrm{star}}\right)$ for $78 \%$ of the RESOLVE-B data set with 

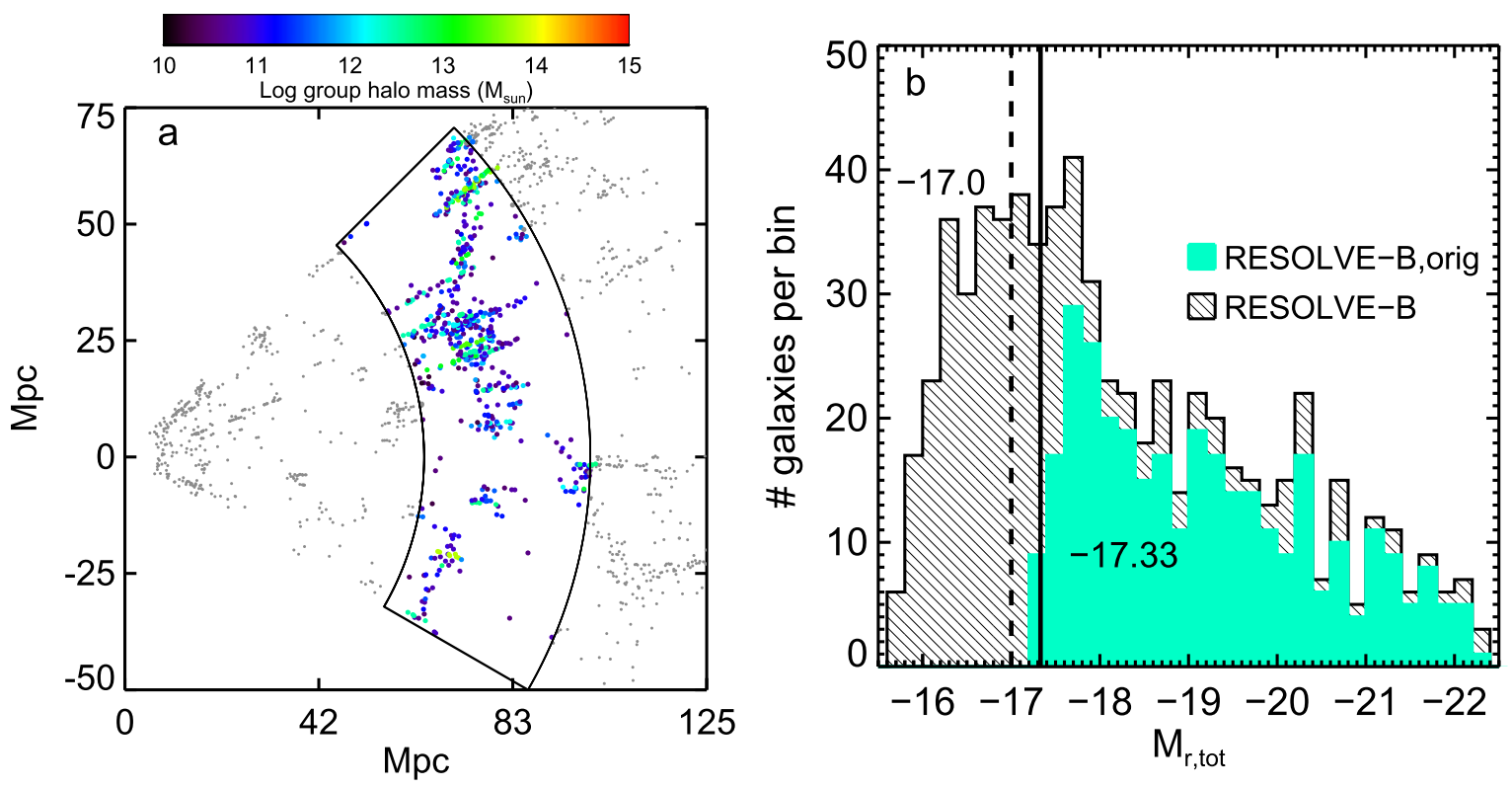

Figure 1. R.A.-cz and luminosity distributions for the Stripe 82 subvolume of RESOLVE described in Section 2.2 (RESOLVE-B). (a) The black outline shows the edges of the $\sim 13,700 \mathrm{Mpc}^{3}$ RESOLVE-B subvolume, which has been collapsed over the narrow decl. range from -1.25 to +1 . 25 . Each point represents an individual galaxy. Galaxies within the RESOLVE volume are color coded according to group halo mass (purple for galaxies in low-mass halos up to green for galaxies in RESOLVE-B's highest mass halos). Gray points show galaxies outside the RESOLVE volume. (b) Luminosity distributions for RESOLVE-B (black crosshatch) and RESOLVE-B,orig (solid green) as defined in Section 2.2. The black solid line shows the original SDSS luminosity completeness limit for RESOLVE-B,orig of $M_{r, \text { tot }}=-17.33$ using the reprocessed photometry described in Section 3.1. Additional redshifts (see Section 2.2) allow us to extend the luminosity completeness limit for RESOLVE-B to $M_{r \text {,tot }}=-17.0$, as shown by the black dashed line, albeit with imperfect completeness below the original SDSS redshift survey limit.

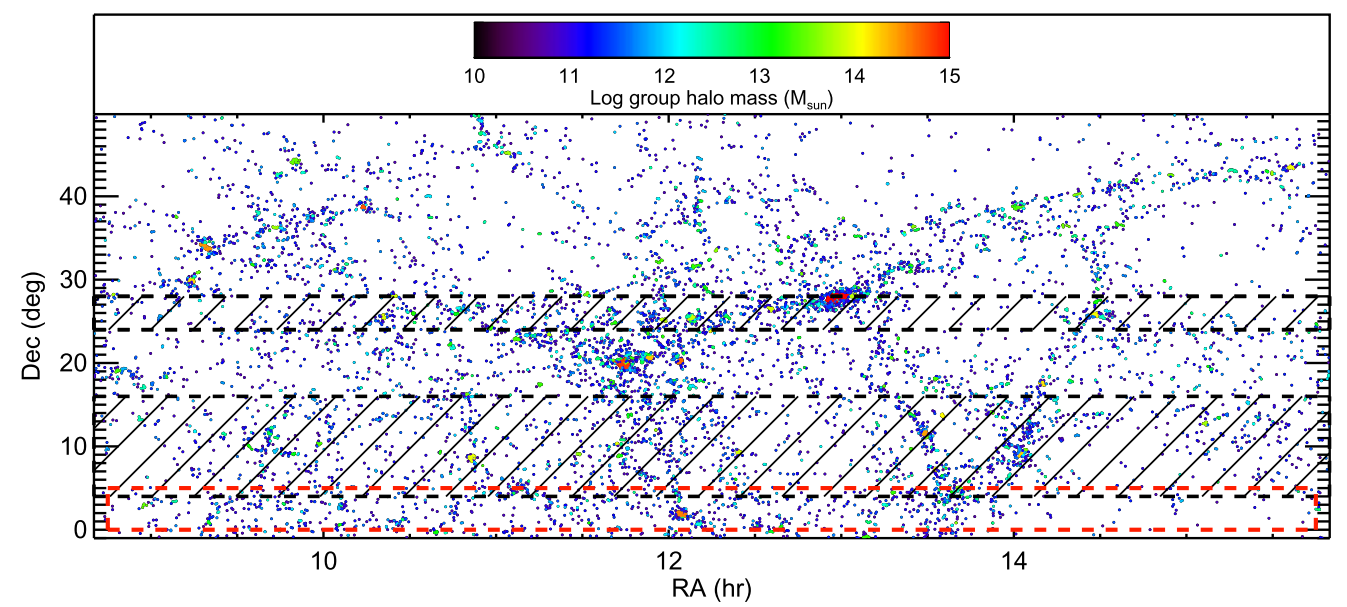

Figure 2. R.A.-decl. distribution of the ECO catalog (M15), which includes galaxies within a $\sim 442,700 \mathrm{Mpc}^{3}$ volume from cz $=3000-7000 \mathrm{~km} \mathrm{~s}{ }^{-1}$, as described in Section 2.3. Each point represents one galaxy, and the galaxies are color coded by group halo mass according to the scale bar at the top (dark blue for low-mass groups and red for the highest mass groups). Overlap with the ALFALFA $21 \mathrm{~cm}$ survey is shown as the black hash-marked region. ALFALFA provides HI detections and upper limits for about one-third of ECO. The RESOLVE-A subvolume footprint, where we have the most complete HI data for ECO, is outlined with the red dashed line.

$M_{r \text {,tot }} \leqslant-17$, with the remaining $22 \%$ of RESOLVE-B galaxies having weak upper limits, confused detections, or no observations (Stark et al. submitted; see also Section 3.3). The HI data statistics for all galaxies in the volume (no restriction on $M_{r \text {,tot }}$ ) yield reliable detections or strong upper limits for $60 \%$ of the RESOLVE-B data set, with the remaining $40 \%$ of RESOLVE-B galaxies having weak upper limits, confused detections, or no observations. In these cases we fill in the HI census using the PGF technique (Section 3.3).

\section{3. $E C O$}

The full ECO catalog, accounting for the buffer region, is a volume-limited data set selected within coordinate and redshift ranges of $8.7 \mathrm{~h}<$ R.A. $<15.82 \mathrm{~h},-1^{\circ}$. $<$ decl. $<+499^{\circ} 85$, and $2530 \mathrm{~km} \mathrm{~s}^{-1}<\mathrm{c} z<7470 \mathrm{~km} \mathrm{~s}^{-1}$ (see Figure 2), containing all known redshifts from the SDSS survey, as well as from the other sources listed in Section 2.1 (M15). The full ECO catalog includes the RESOLVE-A subvolume, which is marked by the red dashed line in Figure 2, and encompasses a volume of $\sim 560,800 \mathrm{Mpc}^{3}$ ( $\sim 41$ times larger than RESOLVE-B), providing a much larger data set for statistical analysis of the galaxy SMF and BMF, but lacking RESOLVE-B's superior completeness. Attempts to fill in completeness through inclusion of archival and RESOLVE redshift data yield a survey $\sim 11 \%$ more complete than the SDSS main redshift survey alone. We take the stated luminosity limit of the SDSS main redshift 
survey as the nominal luminosity completeness limit for ECO. However, additional redshift incompleteness above this inherited luminosity limit remains, which would compromise our mass function analysis (see also Blanton et al. 2005), so we will apply empirical completeness corrections above the nominal luminosity completeness limit in Section 3.6.2.

The volume of the ECO data set used in this work excludes the buffer region and is $\sim 442,700 \mathrm{Mpc}^{3}$, containing 9456 galaxies with $M_{r \text {,tot }}<-17.33$ mag. We limit ECO to galaxies belonging to groups within a cz range of $3000-7000 \mathrm{~km} \mathrm{~s}^{-1}$, allowing the remaining $\mathrm{cz}$ range to serve as a buffer for identifying group members with peculiar velocities outside this range. (A larger buffer of $\mathrm{cz}=1500-12,000 \mathrm{~km} \mathrm{~s}^{-1}$ is used to recover galaxies belonging to the largest groups and clusters with very extended Fingers of God; however, galaxies outside $2530-7470 \mathrm{~km} \mathrm{~s}^{-1}$ lack reprocessed photometry and are treated statistically, as described in more detail in Section 3.5.)

The ECO data set is uniformly covered by single-depth SDSS ugriz and 2MASS JHK imaging and also has 45\% MIS-depth GALEX NUV coverage. Where the ECO catalog overlaps the A-semester of the RESOLVE survey, we use the superior photometric data for RESOLVE (including full MIS-depth GALEX coverage and UKIDSS). We have obtained $21 \mathrm{~cm}$ data from the public ALFALFA40 catalog (Haynes et al. 2011) that cover about one-third of the ECO area and provide detections (including confused detections) and enable calculation of upper limits for 3572 galaxies. Since ALFALFA is a flux-limited survey, $\sim 90 \%$ of the upper limits are weak; that is, they yield gas fractions greater than $5 \%$ of the stellar mass. In the area of overlap with RESOLVE-A, we replace photometry, stellar masses, and HI data with values from RESOLVE-A, including strong upper limits for the HI. Thus the final HI statistics yield nonconfused detections or strong upper limits for $26 \%$ of ECO, with the remaining $74 \%$ of ECO having weak upper limits (14\%), confused detections (6\%), or no observations (54\%). For these ECO galaxies without HI observations, with weak upper limits, or with confused detections, we rely on the PGF technique presented in E15 to provide gas mass estimates (Section 3.3).

\subsection{Densities of RESOLVE-B and ECO}

The data sets used in this work have relatively small volumes, which we have computed using a numerical method and defining the inner and outer radii of the volume using the comoving distance. Because their volumes are small, RESOLVE-B and ECO may be affected by cosmic variance. The primary effect of cosmic variance is on the overall galaxy density in the volume. To gauge this effect for RESOLVE-B and ECO, we compute the number density of galaxies in each volume belonging to the main SDSS redshift survey (to eliminate any difference that is due to overall completeness) within both $M_{r \text {,tot }}<-20$ and $M_{r \text {,tot }}<-21$, and we compare this with the corresponding densities measured by Baldry et al. (2006) for the entire SDSS DR4 volume. ${ }^{6}$ We find that $\log \left(\rho_{<-21}\right)$ and $\log \left(\rho_{<-20}\right)$ for RESOLVE-B are -2.46 and $-2.15 \log \left(\mathrm{Mpc}^{-3}\right)$, and for ECO they are -2.76 and

\footnotetext{
6 The volume calculation in Baldry et al. (2006) has been done with the same cosmological parameters as used in this work $\left(\Omega_{m}=0.3, \Omega_{\Lambda}=0.7\right.$, and $H_{o}=70 \mathrm{~km} \mathrm{~s}^{-1} \mathrm{Mpc}^{-1}$ ). Using our numerical volume calculation method, which determines the volume of the spherical shell defined by the inner and outer radii and then multiplying the shell volume by the ratio of the solid angle subtended by the survey to the solid angle of a sphere, we reproduce their survey volume of $2.3 \times 10^{7} \mathrm{Mpc}^{3}$.
}

$-2.33 \log \left(\mathrm{Mpc}^{-3}\right)$, respectively. For SDSS DR4, Baldry et al. (2006) measure $\log \left(\rho_{-21}\right)$ and $\log \left(\rho_{-20}\right)$ to be -2.8 and $-2.35 \log \left(\mathrm{Mpc}^{-3}\right)$, respectively, at $z \sim 0.02$, which is roughly in the middle of the redshift coverage of the RESOLVE and ECO volumes. Thus, while ECO is similar in density to SDSS DR4, RESOLVE-B is overdense compared to the larger SDSS region, which results in an overall higher normalization of its mass functions.

To gauge whether the relative difference in overall density is within the expected amount of cosmic variance for the RESOLVE-B and ECO volumes, we have considered the cosmic variance recipes of Trenti \& Stiavelli (2008), Driver \& Robotham (2010), and Moster et al. (2011), which are designed for deep high-redshift pencil beam surveys much different from the RESOLVE-B and ECO volumes. These works yield a wide range in cosmic variance estimates, respectively 33\%, 49\%, and $45 \%$ for RESOLVE-B and 16\%, 25\%, and 19\% for ECO. To better assess the cosmic variance of RESOLVE-B and ECO, we use mock galaxy catalogs customized for the RESOLVE-B and ECO volumes (V. Calderon 2016, private communication). We estimate cosmic variance by measuring the total number of galaxies meeting our survey limits in each mock and dividing the standard deviation of those values by the mean value. We obtain cosmic variance estimates of $58 \%$ and $12.5 \%$ for RESOLVE-B and ECO, respectively. These values (along with the results of Driver \& Robotham 2010 and Moster et al. 2011) yield consistent overall number densities in ECO and RESOLVE-B after cosmic variance is taken into account. We defer a more detailed discussion of the cosmic variance within RESOLVE-B and ECO to ongoing work using these custom mock catalogs (J. Cisewski et al. 2016, in preparation).

A secondary effect of cosmic variance is related to the fact that more dense regions tend to have larger halos and structures. We investigate the group halo mass distributions of our two data sets in Section 3.5.2 and find possible evidence for this effect in RESOLVE-B.

\section{DATA AND METHODS}

RESOLVE-B and ECO have largely homogeneous data products with key differences in quality of $\mathrm{HI}$ and degree of completeness. In Section 3.1 we summarize the newly reprocessed photometry from SDSS, 2MASS, UKIDSS, and GALEX, presented in E15 and M15. We then describe the stellar population modeling used to estimate stellar masses from the improved photometry in Section 3.2. In Section 3.3 we review the HI data used in this work as well as the PGF technique from E15, used to predict HI masses for galaxies without HI data or with inadequate HI data. We describe the computation of baryonic masses in Section 3.4. In Section 3.5 we discuss the group finding and halo abundance matching method used to determine halo masses so that we may investigate the mass functions in different group halo mass regimes. Lastly, in Section 3.6 we compare the completeness of RESOLVE-B with estimates of SDSS completeness from the literature, describe our calculation of empirical completeness corrections for ECO, and determine the stellar and baryonic mass completeness limits for RESOLVE-B and ECO.

\subsection{Photometric Data}

For both the RESOLVE and ECO surveys, we use reprocessed photometry from the UV to the near-IR to obtain 
consistent and well-determined total magnitudes, as described in E15 and M15. We use SDSS optical ugriz data (Aihara et al. 2011), NIR $J H K$ and $Y H K$ from 2MASS (Skrutskie et al. 2006) and UKIDSS (Hambly et al. 2008), respectively, and NUV data from the GALEX mission (Morrissey et al. 2007), as well as uvm2 data from the Swift UVOT telescope for 19 RESOLVE-B galaxies lacking MIS-depth NUV data.

We make several key improvements in the photometric reprocessing to obtain consistent photometry with wellcharacterized errors. First we use the improved sky subtraction for SDSS data from Blanton et al. (2011) and our own additional sky subtraction for the 2MASS and UKIDSS data. Second, we apply the same ellipse fits to each band using the sum of the high signal-to-noise ratio $(\mathrm{S} / \mathrm{N})$ gri images to define the ellipses. This approach allows us to determine the PA and axial ratio of the galaxy's outer disk and measure magnitudes even in bands in which catalog photometry has no detection. Third, we compute total magnitudes extrapolated to infinity (not aperture magnitudes) via three nonparametric methods for each band independently, allowing us to obtain systematic uncertainties on the magnitude measurements, key for robust stellar mass estimation through SED fitting (described in Section 3.2). These total magnitude measurements also allow for color gradients, which are explicitly not allowed in the SDSS model magnitude system (see Section 3.1 in E15 for more information).

A key difference between the two surveys is the quality of the data available. For RESOLVE-B, deep optical data from SDSS are available for all galaxies since the Stripe 82 footprint was repeatedly imaged with $\sim 20$ coadds over each area of the sky. Additionally, 98\% of RESOLVE-B galaxies are covered by either MIS-depth ( 1500s) GALEX NUV or Swift uvm2. Finally, $97 \%$ of RESOLVE-B galaxies have deep near-IR photometry in at least one UKIDSS band. For ECO, we are limited to shallow data from SDSS and 2MASS over the entire data set and MIS-depth GALEX for only $\sim 45 \%$ of the data set (M15).

We provide a more in-depth comparison of the reprocessed photometry with the catalog SDSS DR7 photometry in E15. The new photometry yields brighter magnitudes and larger $r_{e}$ values than the SDSS DR7 catalog model and Petrosian calculations. These differences increase for galaxies with larger radii and are in line with the expectations from Blanton et al. (2011), due to improved sky subtraction. We also find generally bluer colors and a less tight red sequence, which we attribute to allowing color gradients (as mentioned, disallowed in the standard SDSS catalog pipeline).

We have demonstrated consistency between RESOLVE-B and ECO photometry in E15, finding rms 0.04 mag between the two in $r$-band magnitude. We attribute small differences to different masking procedures (for RESOLVE, we check all masks by hand, while for ECO, mask checking is only done for autoflagged galaxies) and to the algorithm for merging final magnitudes from our three methods.

\subsection{Stellar Masses}

To calculate stellar masses and colors, we use the SED modeling code described in Kannappan \& Gawiser (2007), Kannappan et al. (2009), and K13, which fits our newly reprocessed total NUVugrizYJHK magnitudes to a grid of stellar population models. With up to 9 or 10 bands of photometric data in ECO and RESOLVE-B, respectively, we can estimate robust stellar masses. In RESOLVE-B we exclude the UKIDSS data if they are flagged due to sky background or image artifact issues (E15). We do not use UKIDSS $J$, and we exclude $H$ - and $K$-band data if they are fainter than 18 and $17.5 \mathrm{mag}$, respectively. We exclude 2MASS JHK data if they are fainter than 16,15 , and $14.5 \mathrm{mag}$, respectively. We exclude any NUV data fainter than $24 \mathrm{mag}$. Galaxy magnitudes fainter than these cuts are unreliable, as determined by an examination of the SED fits. The IR magnitude cuts are also similar to the $10 \sigma$ point source detection limits of 2MASS and the UKIDSS Large Area Survey.

In this work, we consider two model sets to determine how robust the mass function shape is to changes in initial mass function (IMF) and star-formation history. Both model grids consist of an old and a young population, yielding a composite stellar population (CSP). The first model grid (model grid $a$ ) from K13 models the old population with a simple stellar population (SSP) that can range in age from 2 to $12 \mathrm{Gyr}$. The young population of model grid $a$ may be described by continuous star formation that started $1015 \mathrm{Myr}$ ago and turned off between 0 and $195 \mathrm{Myr}$ ago or as a single quenching burst (SSP) of age $360,509,641,806$, or 1015 Myr. The young population can contribute $0 \%-94.1 \%$ of the stellar mass. The second model grid (model grid $b$ ) also uses for its old stellar population an SSP that can range in age from 1.4 to $13.5 \mathrm{Gyr}$. The young population of model grid $b$ is an SSP that can range in age from 5 to $1000 \mathrm{Myr}$ and can contribute $0 \%-64 \%$ of the stellar mass. The model grids are built using the Bruzual \& Charlot (2003) stellar population models. Model grid $a$ uses a Chabrier IMF (Chabrier 2003) with four possible metallicities $(Z=0.004,0.008,0.02$, or 0.05$)$, while model grid $b$ uses a diet Salpeter IMF (Bell \& de Jong 2001) with three possible metallicities $(Z=0.008,0.02$, or 0.05$)$. Both model sets allow for 11 reddening values ranging from 0 to 1.2 that are applied to the young stellar population using the dust law from Calzetti (2001). While the two model sets have a few smaller differences, the largest physical difference between the two model sets is the inclusion of a continuous star-formation mode for the young stellar population in model grid $a$.

For each CSP model in the grid, a stellar mass and a likelihood based on the model fit to the data are computed. We combine the likelihoods over all models, yielding the galaxy's stellar mass likelihood distribution for each galaxy. The nominal value of stellar mass of a galaxy is the median of the stellar mass likelihood distribution. However, for the SMF and BMF calculated later in this work, we use the entire stellar mass likelihood distributions to take into account the large uncertainties on galaxy stellar mass estimates.

From the SED modeling code, we also obtain likelihoodweighted model colors for each galaxy, which are effectively smoothed and $k$-corrected by the model fits. To denote these modeled colors, we use a superscript $\mathrm{m}$ (following the notation from K13, E15, and M15). We have shown in E15 that the RESOLVE-B and ECO color-stellar mass plots are very similar, even though ECO lacks deep optical and IR data and complete NUV coverage.

Comparing the two model sets used in this work, we find an overall offset of $\sim 0.08$ dex (such that model set $a$ masses are smaller) with an rms scatter of $\sigma \sim 0.1$ dex, consistent with K13 and well within the typical uncertainties on stellar mass of 0.15 dex. Model set $a$ is designed to mimic the essential 
features of more complex multiburst star-formation histories (e.g., Salim et al. 2007) with reduced computational demand. We note that the less physically motivated model set $b$ from Kannappan et al. (2009) yields extremely similar results. As a gauge of consistency with literature mass estimates, we note that the slightly altered $b$ model set described in Kannappan et al. (2009) produces stellar masses consistent with those from Kauffmann et al. (2003a), which have also been demonstrated to be consistent with the stellar masses from Salim et al. (2005). All of these comparisons yield an rms scatter of $\sim 0.1$ dex. In contrast, a comparison of masses from an earlier version of model set $b$ described in Kannappan \& Gawiser (2007) with masses estimated with the $g-r$ versus $M_{*} / L_{K}$ relation of Bell et al. (2003b) found that the Bell et al. (2003b) masses are offset toward higher mass by $\sim 0.2$ dex for high-mass galaxies and $\sim 0.3$ dex for low-mass galaxies. Roediger \& Courteau (2015) also find that the Bell et al. (2003b) mass scale and mass-to-light ratios are different from other stellar mass systems.

\subsection{HI Masses}

To measure baryonic masses, we must include the cold neutral gas, the dominant component of which is typically found in atomic hydrogen (although, for large spiral galaxies, molecular gas may dominate; see Figure 8(a) of K13). The HI masses and limits for RESOLVE and ECO come from both the blind $21 \mathrm{~cm}$ ALFALFA survey (Haynes et al. 2011) and from new pointed observations with the Robert C. Byrd Green Bank Telescope (GBT) and Arecibo telescopes (Stark et al. submitted). All galaxies have optical diameters much smaller than the smallest beam size of these telescopes (3!.5 for ALFALFA). Additionally, because of missing data, we estimate masses for a significant portion of the ECO catalog (and a small percentage of RESOLVE-B) using the PGF technique described in E15. To account for the contribution from helium to the cold neutral gas mass, we multiply the HI mass by $1.4: M_{\mathrm{gas}}=1.4 M_{\mathrm{HI}}$.

\subsubsection{RESOLVE-B Gas Inventory}

RESOLVE-B has $21 \mathrm{~cm}$ data from the ALFALFA survey covering the northern $0^{\circ}-1.25$ strip. Data reduction and source extraction have been performed according to Haynes et al. (2011). Since the ALFALFA survey is flux-limited with a fixed HI mass sensitivity of $\sim 10^{9} M_{\odot}$ at RESOLVE redshifts, many of these northern targets have upper limits weaker than our desired goal $\left(M_{\mathrm{gas}}<0.05 M_{\text {star }}\right)$. To fill in the southern Stripe 82 strip and obtain deeper data for these weak upper limits, we have obtained pointed observations with the GBT and Arecibo telescopes for 385 galaxies in RESOLVE-B, aiming for detections with $\mathrm{S} / \mathrm{N}>10$ or strong upper limits (Stark et al. submitted; see also E15). RESOLVE's HI survey only targets galaxies brighter than $M_{r \text {,tot }}=-17.0$ or that have predicted $M_{\text {bary }}>10^{9.0} M_{\odot}$, so many lower-mass galaxies (below our completeness limits) in RESOLVE-B have no HI data.

To measure HI masses and upper limits, we use the algorithms described in K13 and Stark et al. (submitted). Confused sources are determined based on the telescope used for their measurement; we use a search radius of $4^{\prime}$ for the ALFALFA smoothed resolution element, $9^{\prime}$ for the GBT, and 3.5 for the Arecibo pointed observations. We also perform deconfusion of the HI profiles when possible, as described in
Stark et al. (submitted), which builds on techniques described in K13.

For this work (as in E15), we consider an HI detection acceptable if the $\mathrm{HI}$ detection has $\mathrm{S} / \mathrm{N}>5$. If the HI detection is confused, we use the deconfused HI data if the systematic uncertainty is $<25 \%$ of the deconfused $\mathrm{HI}$ mass, as this value is not significantly worse than the error on our weakest $\mathrm{S} / \mathrm{N} \sim 5$ detections. HI upper limits are considered strong if $M_{\text {gas }}<0.05 M_{\text {star. }}$. For galaxies not meeting these requirements, we use gas mass estimates as described in Section 3.3.3.

In RESOLVE-B, limiting the data set at $M_{r \text {,tot }}<-17 \mathrm{mag}$, currently 274 galaxies have unconfused detections and an additional 32 detections have been successfully deconfused. Along with the 74 strong upper limits that yield $M_{\text {gas }}<0.05 M_{\text {star }}, 78 \%$ of RESOLVE-B galaxies have HI data meeting our requirements. Of the remaining 22\%, 34 galaxies cannot be deconfused, three have not been observed in $\mathrm{HI}$, four have unreliable detections with $\mathrm{S} / \mathrm{N}<5$, and 66 have weak upper limits. For the entire RESOLVE-B data set, not restricted to $M_{r \text {,tot }}<-17,334$ galaxies have unconfused or deconfused detections and 74 galaxies have strong upper limits, yielding $60 \%$ of the full RESOLVE-B data set with HI data meeting our requirements. For the remaining $40 \%$ of galaxies, 44 are impossible to deconfuse, 95 have not been observed in HI, eight have low S/N detections, and 124 have weak upper limits.

\subsubsection{ECO Gas Inventory}

Within the ECO catalog, there is a region that overlaps with the ALFALFA40 public catalog (see Figure 2), allowing us to obtain HI detections for galaxies with $\mathrm{HI}$ masses $>10^{9} M_{\odot}$. We calculate HI upper limits as in Stark et al. (submitted) and K13 using the typical declination-dependent rms from ALFALFA. We obtain upper limits for $\sim 40 \%$ of the ECO-ALFALFA cross-matched data set, but only $\sim 11 \%$ of these limits are strong limits $\left(M_{\text {gas }}<0.05 M_{\text {star }}\right)$. Confused sources are flagged if there are neighboring galaxies within the smoothed resolution element of $4^{\prime}$. For ECO, we cannot attempt to extract better fluxes in these cases, which are treated as if they do not have HI data. Confused sources account for $15 \%$ of the ECO-ALFALFA cross-matched catalog.

Within the region of ECO overlapping RESOLVE-A, we substitute RESOLVE HI data from Stark et al. (submitted). The RESOLVE-A footprint has been completely covered by ALFALFA, and we have followed up with GBT and Arecibo pointed observations as in RESOLVE-B, obtaining reliable $\mathrm{HI}$ detections or strong upper limits for $76 \%$ of galaxies with $M_{r, \text { tot }}<-17.33$ (Stark et al. 2016, E15).

Including the RESOLVE-A data with the ECO-ALFALFA regions produces an inhomogeneous HI data set. For this work, however, we wish to include as much real $\mathrm{HI}$ data as possible to measure the BMF. Out of the full ECO data set, 5126 galaxies $(\sim 54 \%)$ have not been observed in HI. Of the 4330 galaxies with HI observations, 2148 have reliable detections (including 34 successfully deconfused HI profiles), 247 galaxies are strong upper limits, 1331 galaxies are weak upper limits, 16 have low $\mathrm{S} / \mathrm{N}$ detections, and 588 galaxies cannot be deconfused. Thus $\sim 75 \%$ of ECO requires PGF estimates.

\subsubsection{Estimating Gas Masses}

We require complete $\mathrm{HI}$ data for RESOLVE-B and ECO to measure the BMF. Thus we turn to the PGF technique to 
empirically estimate gas masses for galaxies in RESOLVE-B and ECO that have not been observed, or for which we have not obtained a reliable detection or strong upper limit. We use the probability density field approach to the PGF technique presented in E15, which uses RESOLVE-A as a calibration data set.

The probability density field method given in E15 fits a twodimensional (2D) model to the density field of log gas-to-stellar mass ratio, $\log (\mathrm{G} / \mathrm{S})$, versus a linear combination of color and axial ratio $(b / a)$, which we call "modified color" or $m c$. From this 2D model it is possible to construct a probability distribution of $\log (\mathrm{G} / \mathrm{S})$ for each galaxy given its color and axial ratio. $^{7}$

The calibration from E15 is ideal for this work for three reasons. First, the PGF calibration from E15 provides a probability distribution in $\log (\mathrm{G} / \mathrm{S})$ for each galaxy, making it easy to integrate into the statistical analysis of the SMF and BMF discussed in Section 4. Second, because the 2D model is defined to include the population of HI upper limits, we can use this calibration even for red quenched galaxies for which colorlimited versions of the PGF calibration break down (see Section 4 of E15). Lastly, the calibration data set, RESOLVE$\mathrm{A}$, is also a volume and absolute $r$-band magnitude-limited survey with a selection similar to RESOLVE-B and ECO.

This last point about using a similarly selected data set for the calibration is key because the prediction values will change if we use a differently selected calibration sample. Since RESOLVE-B and ECO are volume-limited, they are dominated by gas-rich, low-mass galaxies. Other calibrations in the literature are not based on volume-limited data sets and thus do not predict gas masses well for the low-mass galaxies in RESOLVE-B and ECO. In E15, we show that other PGF calibrations underestimate (Catinella et al. 2013; K13) or overestimate (Huang et al. 2012) the actual $\log (\mathrm{G} / \mathrm{S})$ of galaxies at a given color.

A limitation of the 2D model from E15 is that it is calibrated to work on data with all values of $\log (\mathrm{G} / \mathrm{S})$ below -1.3 set equal to $-1.3(\sim \log (0.05))$. Thus we can only predict a galaxy's gas content down to $5 \%$ of its stellar mass, even if we know the upper limit to be lower. The minimum baryonic mass for galaxies with PGF gas mass estimates is therefore $1.05 \times M_{\text {star }}$, which in $\log$ space adds only 0.02 dex to the stellar mass. This shift is much smaller than the typical bin size used in this work $(0.2 \mathrm{dex})$, meaning that we do not have to worry about any significant effect on the mass functions.

\subsection{Baryonic Masses}

To compute baryonic masses, we perform a "pseudoconvolution" of the full stellar mass distributions that are provided by the SED fitting code (see Section 3.2) and the HI likelihood distributions built from either the $\mathrm{HI}$ data or the PGF calibration chosen for each galaxy, yielding a full baryonic mass likelihood distribution for each galaxy. We then take the median of the baryonic mass likelihood distribution to be the nominal baryonic mass of the galaxy but use the full distribution in constructing the mass functions in this work (just as we do for stellar mass). Below we describe the decision tree determining which PGF calibration is chosen for each

\footnotetext{
7 See E15 for more detail on the PGF distributions. IDL and Python codes to generate $\log (\mathrm{G} / \mathrm{S})$ distributions for a given color or modified color are provided at https://github.com/keckert7/codes/.
}

galaxy that requires gas mass estimation. Then we detail the pseudoconvolution algorithm.

\subsubsection{Choice of PGF Estimator}

In choosing the PGF estimator, we prefer to use those calibrations that include colors with the longest baselines, since typical magnitude errors of $\sim 0.05 \mathrm{mag}$ can change the $\log (\mathrm{G} / \mathrm{S})$ value significantly in shorter baseline calibrations. For example, $(g-r)^{m}$ has a prediction baseline of $\sim 0.5 \mathrm{mag}$, while $(u-J)^{m}$ has a prediction baseline of $1.6 \mathrm{mag}$. However, the near-IR colors that provide the longest baselines may be suspect or nonexistent if the galaxy is too faint to be detected or the UKIDSS photometry is flagged. To decide whether the $(u-J)^{m}$ color is reliable enough for use in the PGF relation, we compare the SED-modeled $(u-Y)^{m}$ and $(u-J)^{m}$ colors with the $(u-Y)_{90}$ and $(u-J)_{90}$ aperture colors measured within the $90 \%$ light radius in the $r$ band. If either the $(u-Y)^{m}$ or $(u-J)^{m}$ SED-modeled and aperture colors agree within $2 \sigma$ of the general relation, we proceed with using the modified $(u-J)^{m}$ color (including $b / a$ ) PGF calibration. If not, we move on to using the PGF calibration based on modified $(u-K)^{m}$ color, performing the same analysis (using both 2MASS and UKIDSS $K)$ of comparing $(u-K)^{m}$ and $(u-K)_{90}$ to determine whether the photometry is acceptable. In the case that both $(u-J)^{m}$ and $(u-K)^{m}$ are unsuitable, we revert to the PGF calibration based on modified $(u-r)^{m}$ color, performing the same comparison between $(u-r)^{m}$ and $(u-r)_{90}$. Lastly, for the handful of galaxies with both unreliable $u$ and unreliable 2MASS/UKIDSS, we use the modified $(g-r)^{m}$ color PGF relation, which provides the shortest baseline. Of RESOLVE-B (ECO) galaxies requiring gas mass estimates, 95\% (91\%) use the modified $(u-J)^{m}$ calibration, $3 \%(4 \%)$ use the $(u-K)^{m}$ calibration, $1 \%(2 \%)$ use the modified $(u-r)^{m}$ calibration, and $1 \%(3 \%)$ use the $(g-r)^{m}$ calibration.

For galaxies that have been identified as confused (without possibility of deconfusion), have low $\mathrm{S} / \mathrm{N}$ detections, or have not been observed in HI, we use the PGF estimator $\log (\mathrm{G} / \mathrm{S})$ distribution to perform the baryonic mass likelihood distribution calculation described in Section 3.4.2. For galaxies with weak upper limits, we also use the selected $\log (\mathrm{G} / \mathrm{S})$ distribution, but we cut off the distribution at the weak upper limit value and renormalize the distribution (see Figure 3(b) inset).

\subsubsection{Calculation of Baryonic Masses}

To compute baryonic masses, we start by constructing the stellar mass likelihood distribution for each galaxy. The SED fitting code outputs a mass and likelihood for each model in the grid (model sets $a$ and $b$ contain 26,932 and 9855 models, respectively). The final stellar mass likelihood distribution for each galaxy is binned in 0.01 dex intervals, much smaller than the typical $1 \sigma$ width of the distribution of $\sim 0.25$ dex. Examples of stellar mass likelihood distributions are shown in red in Figure 3.

For galaxies with clean or successfully deconfused HI detections, we model the gas mass likelihood distribution as a Gaussian with $\mu$ equal to the gas mass and $\sigma$ equal to the systematic uncertainty on the gas mass measurement. Two examples of the gas mass likelihood distributions for $\mathrm{HI}$ detections are shown in blue in Figure 3(a). We resample the stellar and gas mass likelihood distributions in linear spacing 

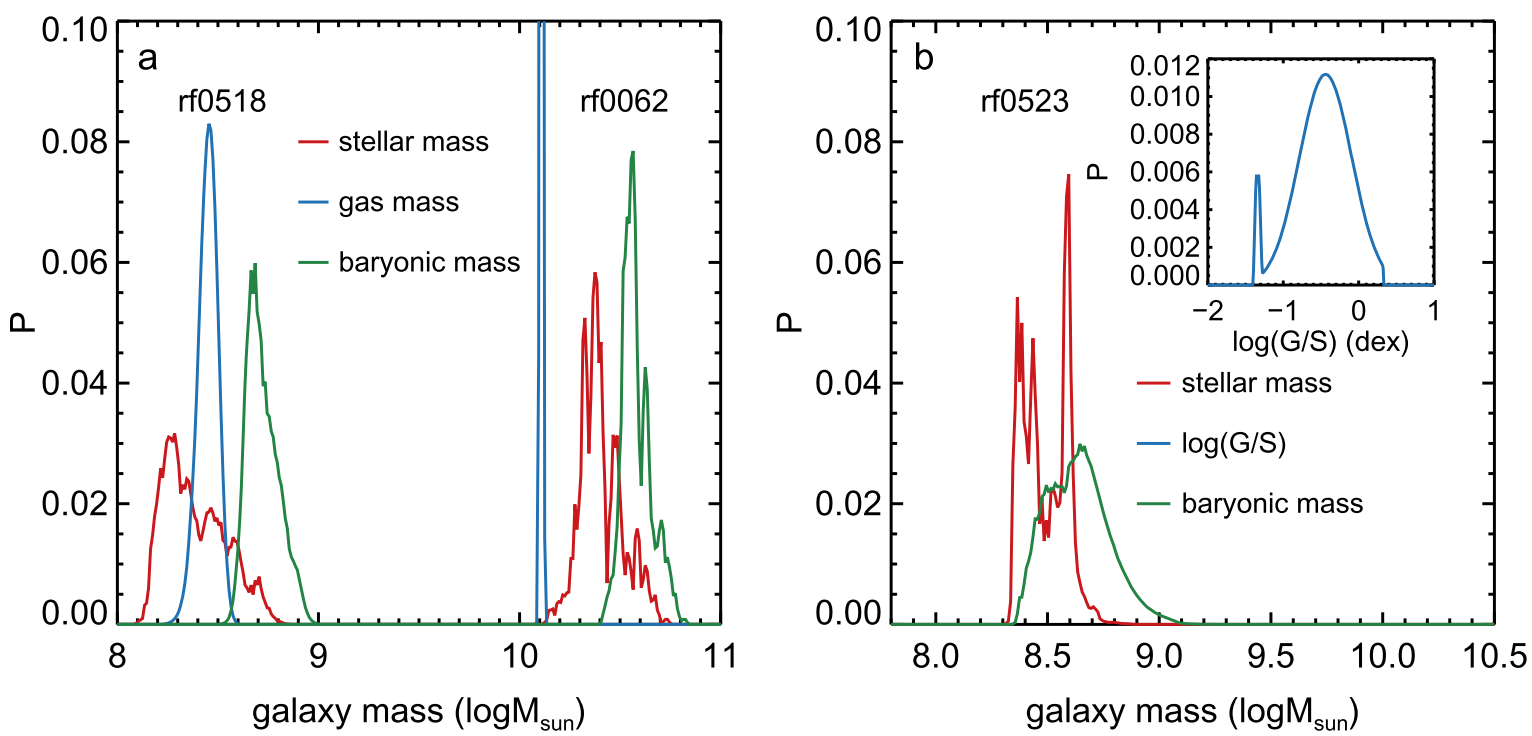

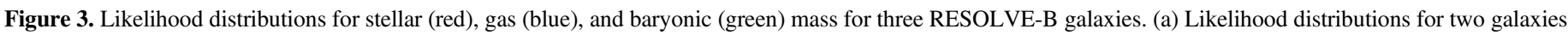

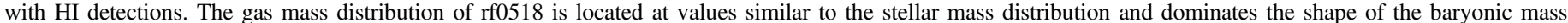

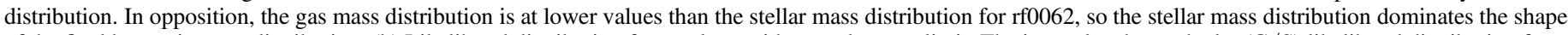

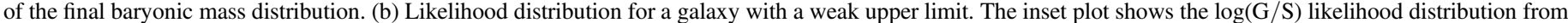

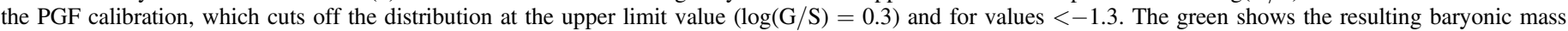
likelihood distribution.

with $\Delta M_{\text {star }}=\Delta M_{\text {gas }}$, set equal to a fraction between 0.01 and 0.5 of the minimum stellar or gas mass with likelihood $>1 \mathrm{e}-4$ (keeping the spacing as small as possible without causing the calculation to be too time consuming). We then determine the combined likelihood for each stellar and gas mass combination in linear mass units from their linearly spaced likelihood distributions. Summing up the likelihoods for all resulting baryonic masses, we obtain a baryonic mass likelihood distribution.

For galaxies with weak upper limits, low $\mathrm{S} / \mathrm{N}$ detections, no HI observations, or severely confused detections, we use the $\log (\mathrm{G} / \mathrm{S})$ distributions from the PGF calibration described in Section 3.4.1. An example of a $\log (\mathrm{G} / \mathrm{S})$ distribution for $a$ weak upper limit is shown in the inset of Figure 3(b). To find the baryonic mass likelihood distribution for galaxies, we resample the stellar mass likelihood distribution into linear spacing with $\Delta M_{\text {star }}$ equal to a fraction between 0.01 and 0.5 of the minimum stellar mass value having likelihood $>1 \mathrm{e}-4$. For each possible stellar mass, we compute a new spacing for the $\log (\mathrm{G} / \mathrm{S})$ distribution such that $\Delta M_{\text {gas }}=\Delta M_{\text {star. }}$. The likelihood and baryonic mass are then computed at each possible stellar mass and $\mathrm{G} / \mathrm{S}$ value. We then sum the likelihoods at each baryonic mass to produce the baryonic mass distribution.

Finally, for galaxies with strong upper limits, we set the likelihood in $\log (\mathrm{G} / \mathrm{S})$ to be 1 at the upper limit value of $\log (\mathrm{G} / \mathrm{S})$. Since for strong upper limits this value can be at most 0.05 , the baryonic mass will be at most 0.02 dex larger than the stellar mass.

The final baryonic mass likelihood distribution for each galaxy comes from the array of likelihoods and baryonic masses output by these procedures and is binned up finely into 0.01 dex bins. Three examples are shown in green in Figure 3 for the different cases. To compute single-value baryonic masses, we determine the median value of the resulting baryonic mass likelihood distribution for each galaxy, just as for stellar mass.

\subsection{Group Identification and Halo Masses}

To investigate the SMF and BMF in different group halo mass regimes, we associate the galaxies in the RESOLVE-B and ECO data sets with groups using the FOF group-finding algorithm from Berlind et al. (2006) following the algorithm described in M15. We are able to use this algorithm because our data sets are limited on absolute magnitude and are volumelimited. In the following sections, we describe the choice of linking lengths and the group finding and halo mass assignment. We also compare the halo mass distributions for RESOLVE-B and ECO and examine the relationship between galaxy stellar and baryonic mass and halo mass.

\subsubsection{Choice of Linking Lengths}

The FOF algorithm links galaxies that lie within a cylinder defined by a tangential linking length (in projected physical distance) and a line-of-sight linking length (in cz space), which are determined by the mean spacing between objects in the volume. In Berlind et al. (2006), the best tangential and line-ofsight linking lengths are determined to be 0.14 and 0.75 times the mean spacing between galaxies. Using mock catalogs, the linking lengths are optimized to reproduce the multiplicity function and projected sizes of groups with $>10$ galaxies.

However, we prefer a larger line-of-sight linking length and smaller tangential linking length for this work. Larger line-ofsight linking lengths are better for recovering the full Fingers of God of groups. For this reason, an analysis of the mock catalog data finds that a line-of-sight linking length of 1.3 best optimizes the recovery of group velocity dispersions (A. Baker 2014, B.S. honors thesis ${ }^{8}$ ). We also find that using a tangential linking length of 0.14 overlinks low- $N$ groups: singleton galaxies are linked into false pairs and triplets. We have used the mock galaxy catalog described in M15 to compare the distribution in distances between galaxies in pairs and triplets

\footnotetext{
8 http://resolve.astro.unc.edu/pdf/ashbake_thesis3.0.pdf
} 
with the distribution of distances between single galaxies and their nearest neighbors. Based on this analysis, a tangential linking length of 0.07 minimizes the breaking up of truly paired galaxies $(<5 \%)$, while preventing the overgrouping of truly single galaxies. The independent analysis of linking-length parameter space provided by Duarte \& Mamon (2014) suggests optimal tangential and line-of-sight linking lengths of 0.07 and 1.1 for studies of galaxy properties in the context of environment. Their study showed that these linking lengths result in low group merging fractions across all group halo masses, although they do result in high fragmentation for the largest groups (of which there are relatively few in RESOLVE and ECO). Overall galaxy completeness and reliability are found to be high using these linking lengths to find groups. These linking lengths are also similar to those found in the analysis of Robotham et al. (2011), which determined that while low- $N(N<5)$ groups do suffer from contamination, their integrated group luminosities are not strongly affected. An analysis of mock galaxy catalogs customized for the RESOLVE and ECO surveys shows that these linking lengths yield high purity and completeness $(>0.75)$ for centrals and satellites in low-mass, low- $N$ groups. The purity and completeness decrease for higher mass halos to $\sim 0.5$ (V. Calderon 2016, private communication). Given these results, we adopt tangential and line-of-sight linking lengths 0.07 and 1.1 to create our group catalog. These linking lengths were also used in M15.

\subsubsection{Group Finding and Halo Mass Assignment}

To find groups in RESOLVE, we use the same general algorithm described in M15 and outlined below. For ECO, we perform the group finding on the full data set over the redshift range from 2530 to $7470 \mathrm{~km} \mathrm{~s}^{-1}$, limiting the data set to galaxies brighter than $M_{r \text {,tot }}=-17.33$ mag. We also construct a RESOLVE-B-analog data set from ECO, including galaxies down to $-17.0 \mathrm{mag}$. We create this analog data set to determine the physical linking lengths for the RESOLVE-B data set, for which the volume is too small and subject to cosmic variance to determine the linking lengths dynamically. For RESOLVE-B, we perform the group finding with these fixed physical linking lengths for galaxies brighter than $M_{r, \text { tot }}=-17.0 \mathrm{mag}$ and over the range $4250-7250 \mathrm{~km} \mathrm{~s}^{-1}$, allowing for a $250 \mathrm{~km} \mathrm{~s}^{-1}$ buffer on either side of the subvolume. In Figure 4(a), we show the resulting group luminosity distributions for ECO and RESOLVE-B.

Halo masses are inferred from the total group $r$-band luminosity using halo abundance matching between the identified groups and the theoretical group HMF from Warren et al. (2006), assuming cosmological parameters consistent with WMAP5 (Dunkley et al. 2009). The algorithm assumes zero scatter between group luminosity and group halo mass (this scatter has been estimated to be in the range $\sigma_{L}=0.13-0.17$ at a fixed halo mass for central galaxies; see Cooray 2006; Yang et al. 2008; More et al. 2009). Because the RESOLVE-B volume is small and subject to cosmic variance, we fit a spline to the abundance-matching result (group $L_{r}$ versus halo mass relation) from the RESOLVE-B-analog version of the ECO catalog and use the fit to assign halo masses to the groups in RESOLVE-B. Therefore, the halo masses assigned to the ECO and RESOLVE-B groups are consistent, as shown in Figure 4(b).
Note that, in this work, we have not performed any correction to the group halo masses, unlike M15. In M15, group finding and halo abundance matching were performed for both ECO and a large mock catalog. The mock catalog was used to assess whether there was an offset between the assigned group halo masses and the true halo masses in the simulation. An offset of -0.15 dex was found and applied to the ECO group halo masses. Further investigation has revealed, however, that the overall simulation used for the mock catalog is underdense compared to ECO (shown to be similar to the overall SDSS in Section 2.4), and this underdensity leads to groups being assigned larger masses than their true masses in the simulation. Performing the comparison of assigned to true group halo masses within a subvolume of the mock catalog that has density similar to ECO results in no offset between the true and assigned group halo masses. Thus we do not apply any offset, and our group halo masses are $\sim 0.15$ dex larger than those reported in the M15 ECO catalog. We refer to these masses as halo masses throughout this paper; however, we emphasize that errors in group finding can cause significant scatter $(\sigma \sim 0.1)$ between our estimated masses and the true masses of the underlying halos, in addition to the neglected intrinsic scatter between $L_{r \text {,tot }}$ and group halo mass.

To test the robustness of our galaxy mass functions computed in different halo mass bins (see Section 5.3), we have also performed halo abundance matching based on group stellar mass rather than group $r$-band luminosity. The results shown in Section 5.3 are not affected by whether we use group luminosity or group stellar mass for halo abundance matching.

In Figure 5(a) we compare the HMFs for RESOLVE-B (black striped histogram) and ECO (green solid histogram) normalized by each data set's respective number of halos. The ECO group HMF is smooth by definition as it is directly matched to the HMF of Warren et al. (2006). RESOLVE-B has a noisier distribution since we assign group halo masses using the group luminosity to group halo mass relation for the RESOLVE-B-analog version of ECO. While RESOLVE-B has no groups more massive than $10^{13.5} M_{\odot}$, it does have an overabundance of halos of mass $\sim 10^{12.5} M_{\odot}$ and $10^{13.5} M_{\odot}$. Since RESOLVE-B is overdense, as described in Section 2.4, its HMF is slightly elevated over that of ECO. Although RESOLVE-B does not contain any clusters, the fact that it is overdense may contribute to the large number of intermediate and large group halos in RESOLVE-B, because more dense areas tend to have larger structures.

We also look at the relationship between galaxy stellar or baryonic mass and group halo mass in Figure 5(b). Central galaxies are determined to be the brightest galaxy in a group and are denoted by larger symbols. Centrals show a monotonic relationship with halo mass, which we model as a function of two power laws, as given in Equation (1):

$$
M_{\mathrm{gal}}=\phi_{0} \frac{\left(\frac{M_{\mathrm{grp}}}{M_{0}}\right)^{\alpha}}{\left(x_{0}+\frac{M_{\mathrm{grp}}}{M_{0}}\right)^{\beta}} .
$$

Using MPFITFUN (an IDL code that implements a Levenberg-Marquardt least squares fit; see Moré 1978, pp. 105-116, and Markwardt 2009), we fit this model to the central stellar and baryonic mass to halo mass relationships for RESOLVE-B and ECO. The parameters of the fits are given in 

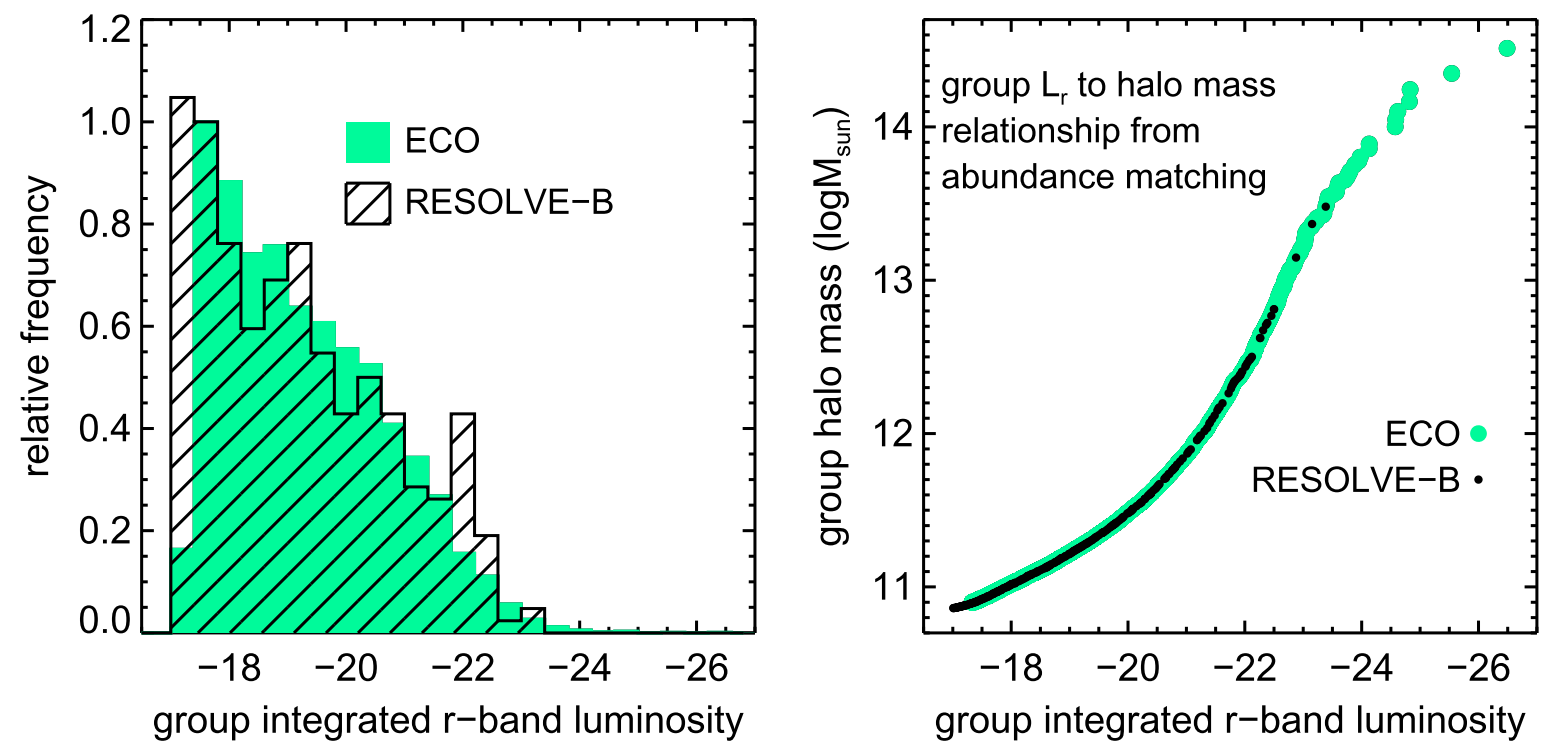

Figure 4. (a) The frequency distributions of group integrated $r$-band luminosity in RESOLVE-B and ECO. The distributions have been normalized by the number of groups in the last complete bin for ECO. The distribution of ECO is much less noisy than that of RESOLVE-B. (b) The relationship of group halo mass to group integrated $r$-band luminosity for ECO and RESOLVE-B determined through abundance matching to the theoretical group HMF from Warren et al. (2006). The relationship for RESOLVE-B is determined by a spline fit to the abundance-matching relation for the RESOLVE-B-analog version of ECO, which extends down to $M_{r, \text { tot }}=-17.0$ mag. Thus, groups of similar luminosity in RESOLVE-B and ECO are matched to the same group halo mass.
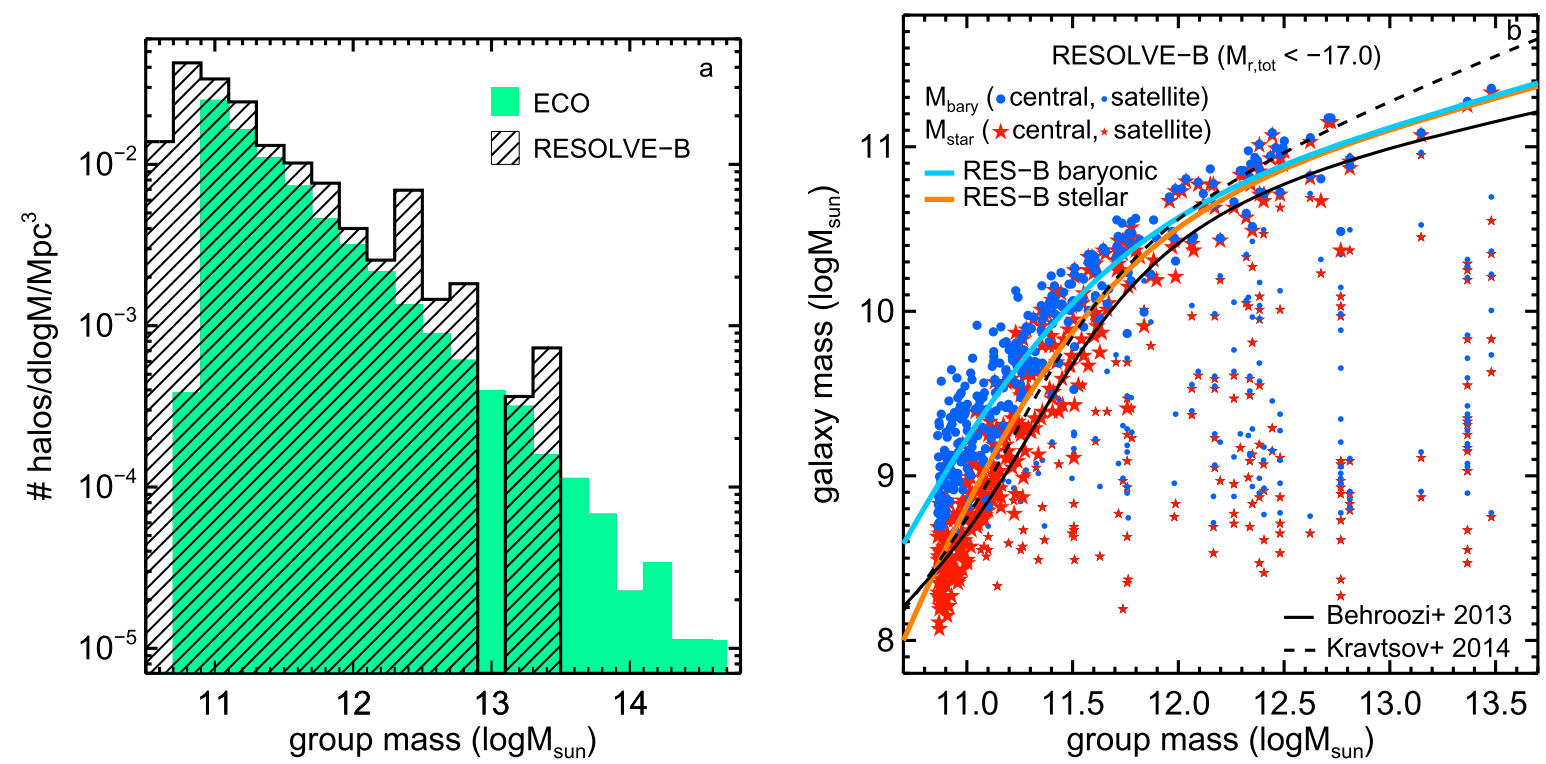

Figure 5. The group halo mass distribution and relationship between galaxy stellar or baryonic mass and group halo mass. (a) RESOLVE-B (black crosshatch) and ECO $\left(M_{r \text {,tot }}<-17.33\right.$; solid green) HMFs using the FOF algorithm of Berlind et al. (2006) to find groups and halo abundance matching to assign masses based on a total group luminosity to halo mass conversion factor (Section 3.5). RESOLVE-B has an overabundance of group halos of mass $\sim 10^{12.5} M_{\odot}$ and $\sim 10^{13.5} M_{\odot}$ and no groups more massive than $10^{13.5} M_{\odot}$, unlike the smoother ECO halo mass distribution. (b) Galaxy stellar or baryonic mass to group halo mass relation for RESOLVEB (limited to galaxies brighter than -17). The stellar and baryonic masses are computed from the median of the likelihood-weighted mass distributions described in Section 3.4.2. The plot shows both the central galaxy stellar (large red stars) and baryonic (large blue dots) masses and the satellite galaxy masses (smaller symbols). The orange and blue solid lines show our fits to the RESOLVE-B central stellar and baryonic mass to halo mass relationships according to Equation (1) (parameters are given in Table 1 along with the similar results for ECO). We find that the central stellar to halo mass relation is in agreement with the central stellar to halo mass relationships from Behroozi et al. (2013) and Kravtsov et al. (2014), splitting the difference at large group halo masses (Section 3.5.2).

Table 1, and the RESOLVE-B stellar and baryonic fits are shown in Figure 5(b) as orange and blue lines, respectively. Our stellar mass to halo mass relationship lies between those of Behroozi et al. (2013) and Kravtsov et al. (2014). (The steeper relationship of Kravtsov et al. 2014 reflects photometry from Bernardi et al. 2013, which recovers extended light around the brightest cluster galaxies.) Below a halo mass of $\sim 10^{12} M_{\odot}$, the baryonic mass of centrals starts to become significantly larger than the stellar mass.
Satellite galaxies are denoted by smaller symbols in Figure 5(b) and are seen cascading down from the central galaxy mass to halo mass relationship. There is no clear relationship between the stellar or baryonic masses of satellites and halo mass, although it is evident that the number of satellites increases for larger halos.

Since the RESOLVE-B data set extends to luminosities fainter than $-17 \mathrm{mag}$, we perform an extra step to determine whether any galaxies with $M_{r \text {,tot }}>-17$ belong to previously 
Table 1

Central Stellar and Baryonic Mass to Group Halo Mass Fit Parameters

\begin{tabular}{|c|c|c|c|c|c|}
\hline Data & $\begin{array}{c}\log \phi_{0} \\
\log \left(M_{\odot}\right)\end{array}$ & $\begin{array}{c}\log M_{0} \\
\log \left(M_{\odot}\right)\end{array}$ & $\alpha$ & $\beta$ & $x_{0}$ \\
\hline RESOLVE-B stellar & $10.73 \pm 0.72$ & $11.85 \pm 2.04$ & $3.49 \pm 0.21$ & $3.14 \pm 0.19$ & $0.32 \pm 1.52$ \\
\hline RESOLVE-B baryonic & $10.69 \pm 0.87$ & $11.77 \pm 2.40$ & $2.67 \pm 0.22$ & $2.31 \pm 0.19$ & $0.39 \pm 2.18$ \\
\hline ECO stellar & $10.50 \pm 0.22$ & $11.55 \pm 0.56$ & $6.56 \pm 0.24$ & $6.16 \pm 0.23$ & $0.24 \pm 0.31$ \\
\hline ECO baryonic & $10.76 \pm 0.23$ & $11.83 \pm 0.68$ & $2.34 \pm 0.05$ & $2.00 \pm 0.05$ & $0.44 \pm 0.68$ \\
\hline
\end{tabular}

identified groups. First, we determine whether the faint galaxy is within the virial radius of a group center. If so, we determine whether the faint galaxy's recessional velocity is within the larger of the line-of-sight linking length or three times the group velocity dispersion from that group center. If the galaxy meets both the radius and velocity criteria, it is matched to the group. If it does not match any group, it is placed in a group by itself and given a halo mass based on the extrapolation of the halo mass to group integrated $r$-band luminosity relation used in abundance matching. Of the 192 galaxies in RESOLVE-B fainter than $-17 \mathrm{mag}, 47$ are associated with identified RESOLVE-B groups and 145 are in halos by themselves.

\subsection{Completeness of Data Sets}

To ensure that we interpret the SMF and BMF correctly, it is important that we understand the stellar and baryonic mass completeness limits of the RESOLVE-B and ECO data sets. In Section 3.6.1, we compare the surface brightness completeness of RESOLVE-B with estimates of SDSS completeness from the literature to show that RESOLVE-B is a highly complete data set. In Section 3.6.2, we present empirical completeness corrections derived for the ECO data set based on RESOLVEB. In Section 3.6.3, we determine the stellar and baryonic mass completeness limits for RESOLVE-B and ECO.

\subsubsection{RESOLVE-B Completeness}

Since RESOLVE-B has the benefit of additional redshift coverage from several sources, we wish to compare its added completeness with the estimated incompleteness of the SDSS main redshift survey determined in Blanton et al. (2005). The SDSS main redshift survey is known to have spectroscopic incompleteness of $\sim 6 \%-10 \%$ because of a mechanical issue limiting the minimum spacing between fibers to $55^{\prime \prime}$ from each other (Blanton et al. 2003b). This incompleteness estimate, however, is limited to galaxies that were targeted for spectroscopic follow-up $\left(m_{r \text {,petro }}<17.77\right)$.

Other sources of incompleteness arise from known issues with the SDSS photometric pipeline that cause galaxies to be omitted as targets in the redshift survey. These problems are oversubtraction of sky around the galaxy, causing the amount of flux to be underestimated (Strauss et al. 2002; Blanton et al. 2011), and "shredding" of galaxies, which means that rather than identifying and measuring the flux for one galaxy, the pipeline breaks up the galaxy into several individual pieces, measuring the flux for each piece (Stoughton et al. 2002). This "shredding" means that no one piece of the galaxy is bright enough to be included in the redshift survey, even if the galaxy is truly bright enough. In addition, low-surface brightness galaxies $\left(\mu_{50}<24.5 \mathrm{mag} \operatorname{arcsec}^{-2}\right)$ were deliberately excluded from spectroscopic follow-up despite meeting the magnitude cut (Strauss et al. 2002).
To gauge the spectroscopic incompleteness caused by photometric pipeline issues that underestimate the flux in galaxies that could otherwise be bright enough to qualify for the redshift survey, Blanton et al. (2005) used simulated galaxies to test the effectiveness of the SDSS photometric pipeline over a range of galaxy surface brightnesses. They took into account galaxies lost via sky oversubtraction and "shredding" to determine the survey completeness as a function of surface brightness. Figure 6(a) shows their spectroscopic incompleteness (or 1-completeness) as a function of surface brightness (black solid line). Green dash-dotted and red dashed lines show the higher survey incompleteness, taking into account a $6 \%$ or $10 \%$ loss that is due to fiber collisions, respectively.

To compare with the results of Blanton et al. (2005), we plot one minus the ratio of the number of galaxies in RESOLVE-B,orig divided by the number of galaxies in the final RESOLVE-B as a function of surface brightness. Because the SDSS photometric measurements are not adequate for many of these additional galaxies, we use our reprocessed photometry and limit both data sets to an $r$-band apparent magnitude of $m_{r \text {,tot }}<17.67 \mathrm{mag}$, which corresponds to $m_{r \text {,petro }}<17.77 \mathrm{mag}$. We then perform a fit between $\mu_{r \text {,petro50 }}$ and $\mu_{r, 50}$ to translate the surface brightness measured in this work to that of the SDSS for comparison with Blanton et al. (2005) (Figure 6(b))

We find that for galaxies with converted Petrosian surface brightnesses brighter than $\sim 20 \mathrm{mag} \mathrm{arcsec}^{-2}$ the added RESOLVE-B completeness is consistent with the results of Blanton et al. (2005); that is, we have recovered the expected number of galaxies lost due to photometric errors and fiber collisions. For galaxies fainter than $\sim 20 \mathrm{mag} \mathrm{arcsec}^{-2}$, we find a higher-than-expected recovery rate of missing galaxies in RESOLVE-B. We treat this result as evidence that the RESOLVE-B data set is as close to complete as possible, making RESOLVE-B a powerful data set for galaxy population studies.

\subsubsection{ECO Completeness Corrections}

The ECO catalog is less complete than RESOLVE-B because of two issues: (1) it does not have as complete of a redshift inventory as RESOLVE-B, although we have added galaxies where available from several sources, and (2) it contains massive groups and clusters (including Coma) for which a substantial number of galaxies may not be included in the volume because of large peculiar velocities. To correct for the first issue, we use empirical completeness corrections based on the galaxy distributions in the $M_{r \text {,tot }}$ versus surface brightness and $M_{r \text {,tot }}$ versus color parameter spaces. To correct for the second issue, we employ the group-finding algorithm on a larger volume to estimate the fraction of galaxies in large groups and clusters missed because of large peculiar velocities. 

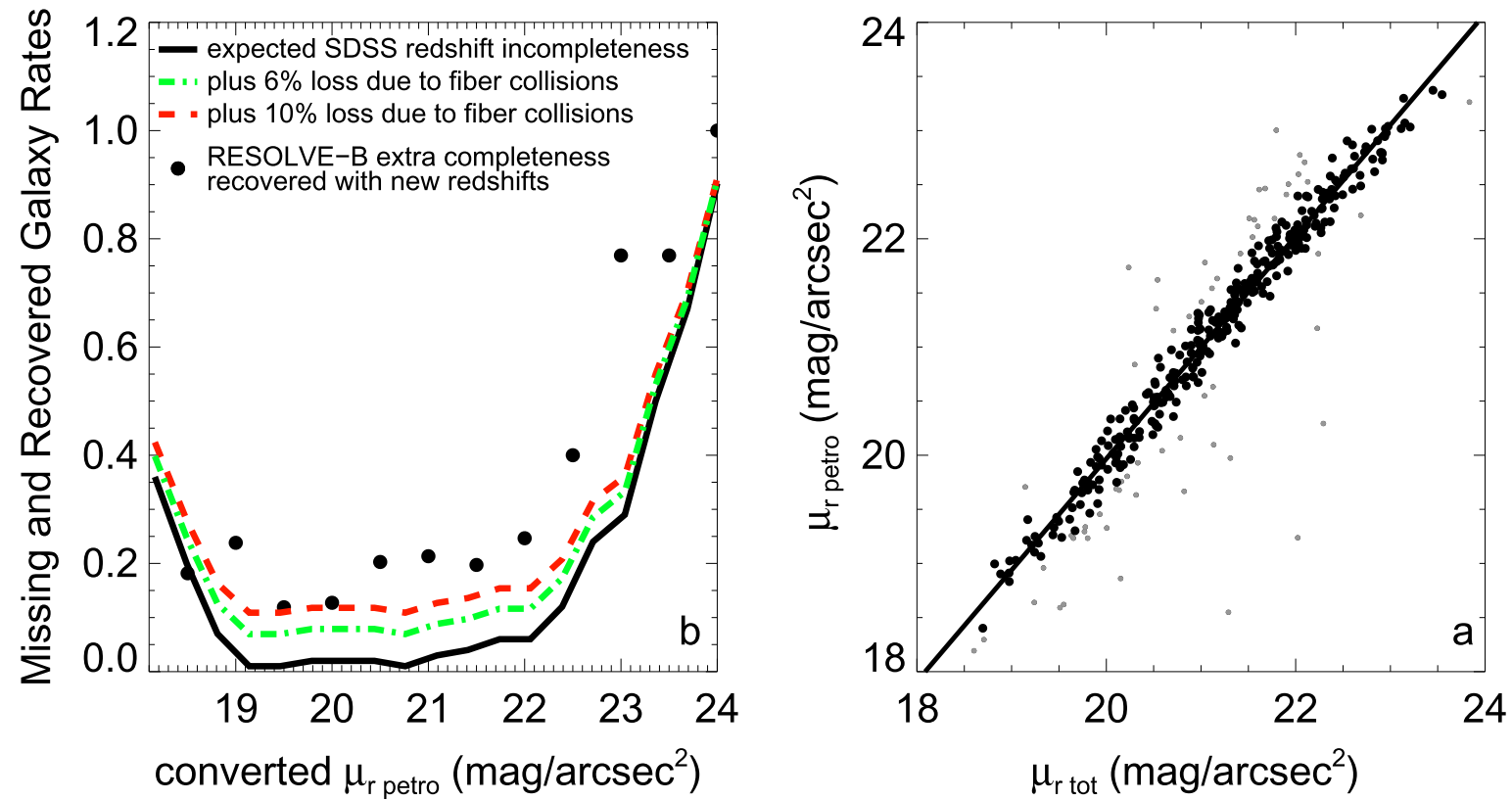

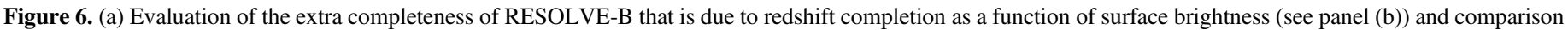

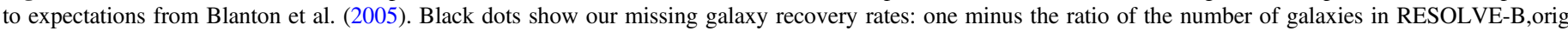

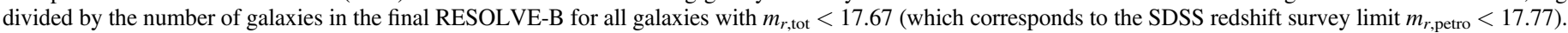

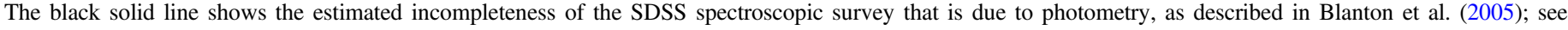

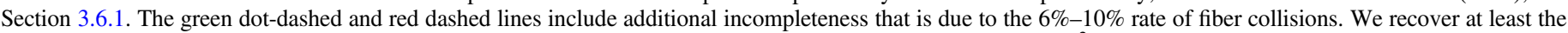

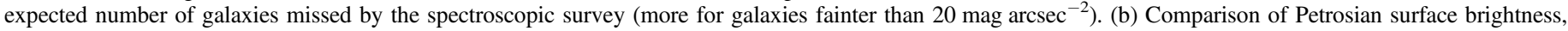

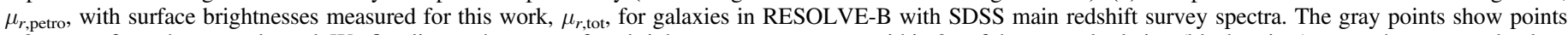

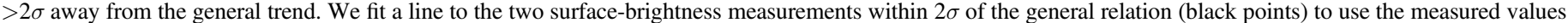

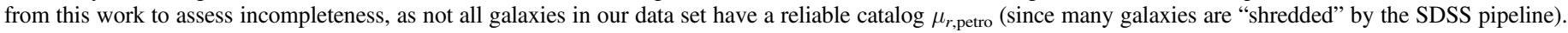

To calculate the empirical completeness corrections, we follow the same methodology as presented in M15 and first construct a base sample for both RESOLVE-B and ECO consisting only of galaxies with SDSS DR7 redshifts, $M_{r \text {,petro }}<-17.23 \mathrm{mag}$, and having local group corrected velocities within the respective velocity ranges of each data set. We call these samples RESOLVE-B-DR7 and ECO-DR7. Second, we construct a sample for both RESOLVE-B and ECO consisting of all available local group corrected velocities within the velocity range for each data set and with $M_{r, \text { tot }}<-17.33 \mathrm{mag}$. We call these samples RESOLVE-BDR7+ and ECO-DR7+. The RESOLVE-B-DR7+ sample is complete, while the ECO-DR7+ sample is only partially complete.

To calculate completeness corrections, we adopt the method described in M15 of adaptively binning each data set above in $M_{r \text {,tot }}$ and $\mu_{r}$ and $M_{r \text {,tot }}$ and $(g-i)^{m}$ parameter space. The adaptive binning starts out with coarse bins and then refines the bin size until no more than $10 \%$ of the data set $(\sim 5$ galaxies for RESOLVE-B, 100 galaxies for ECO) exists in one bin. The irregularly gridded field is then interpolated onto a smooth density field.

The RESOLVE-B recovery rate field is simply the RESOLVE-B-DR7+ field divided by the RESOLVE-B-DR7 field. We cannot apply this RESOLVE-B recovery rate directly to the ECO catalog data, though, since the ECO catalog has also been supplemented by other redshift sources, albeit to a lesser degree. Thus we create the ECO recovery rate field with the ECO-DR7 + field divided by the ECO-DR7 field. The final completeness-correction field that we apply to the ECO catalog is then the RESOLVE-B recovery rate field divided by the ECO recovery rate field. We perform a boxcar smoothing of this final correction field, replacing values if they are $>2 \sigma$ above the mean within a $7 \times 7$ box, and we further do not allow the field to have correction factors below one. To find the completeness correction value for a given galaxy, we evaluate the 2D field at the galaxy's $M_{r \text {,tot }}$ and $\mu_{r}$ or $M_{r \text {,tot }}$ and $(g-i)^{m}$ color. The completeness correction for each galaxy is saved as a weight vector. ${ }^{9}$

The luminosity distribution of the raw ECO data set is shown as a red dash-dotted outline histogram in Figure 7 (normalized to the maximum bin height in the raw ECO data set), and the luminosity distribution for the completeness-corrected ECO using $\mu_{\text {iso }}$ is shown in black (also normalized to the maximum bin height for the raw ECO data set). We find that the $\mu_{\text {iso }}$ and $(g-i)^{m}$ completeness correction fields provide similar corrections for ECO. The completeness-corrected luminosity distribution for ECO agrees much better with the RESOLVE-B luminosity distribution.

To correct for the cluster galaxies whose peculiar motions extend outside the ECO volume, we use the results from M15, who performed group finding on a larger catalog extending from 1500 to $12,000 \mathrm{~km} \mathrm{~s}^{-1}$ using SDSS catalog $r$-band measurements and limiting the absolute $r$-band magnitude to galaxies brighter than $M_{r \text {,petro }}=-18.4 \mathrm{mag}$. The groups in the ECO catalog were cross-matched with groups identified in this larger catalog. The ratio of the number of galaxies with $M_{r \text {, petro }}<-18.4 \mathrm{mag}$ in the larger catalog to the number of galaxies with $M_{r \text {,petro }}<-18.4$ mag in the ECO catalog is used

\footnotetext{
9 We have tested whether dividing our samples into two different halo mass bins affects the resulting completeness correction fields. We found no difference in the resulting completeness corrections, but the small number of RESOLVE-B galaxies may limit our ability to detect any group halo massdependent effects.
} 


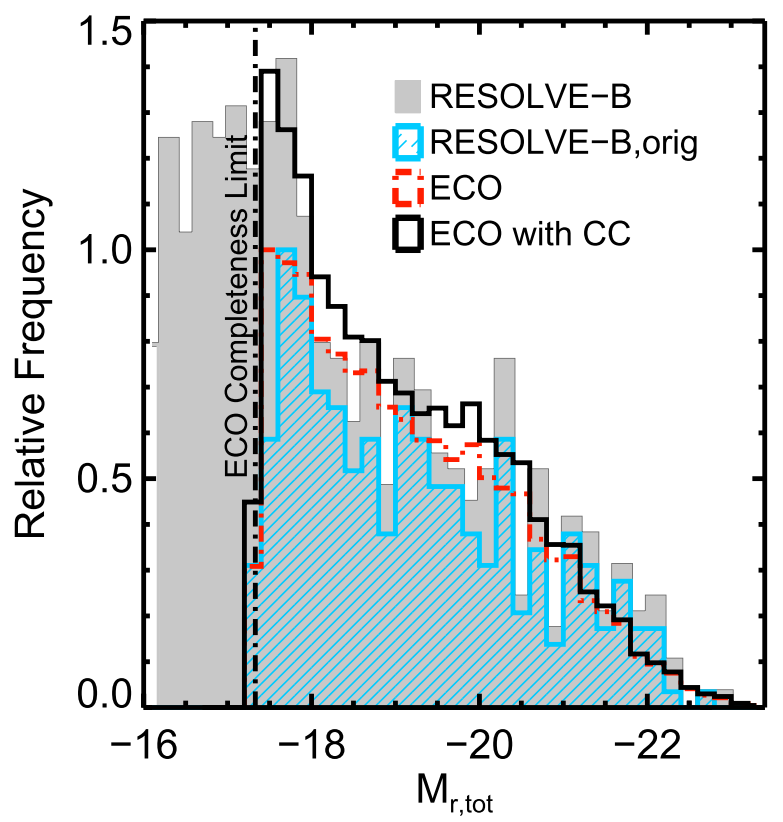

Figure 7. Relative frequencies of $r$-band absolute magnitude for the original and final RESOLVE-B data sets and the raw and completeness-corrected ECO data sets. RESOLVE-B is shown in solid gray and RESOLVE-B,orig in crosshatched blue, where both have been normalized to the maximum bin height in the RESOLVE-B,orig distribution. The raw ECO data set is shown in dashdotted red, and ECO using the $M_{r \text {,tot }}$ versus $\mu_{\text {iso }}$ completeness corrections is shown in solid black (the result is similar when using $(g-i)^{m}$ color). Both histograms have been normalized to the maximum bin height of the raw ECO distribution. We show the distributions normalized to the maximum height of the original data set to emphasize the relative boost factor for ECO at each luminosity and how that compares to the difference between RESOLVE-B,orig and RESOLVE-B. The ECO luminosity completeness limit at $M_{r \text {,tot }}=-17.33$ is shown with a black dash-dotted line.

to find any groups that are missing significant numbers of galaxies. We apply this ratio as the correction factor to galaxies in these groups, even for galaxies fainter than -18.4 mag. Because the reprocessed photometry is not available outside the ECO buffers $\left(<2530 \mathrm{~km} \mathrm{~s}^{-1}\right.$ and $\left.>7470 \mathrm{~km} \mathrm{~s}^{-1}\right)$, the "recovered" galaxies outside the ECO buffers are not included in the final ECO group catalog and are used only to calculate the correction factor. Only three groups are affected by this issue, including the Coma cluster. We correct the masses of these groups by taking into account the missing galaxies' luminosity. The group mass estimate for Coma, though, only changes by 0.06 dex (M15).

\subsubsection{Stellar and Baryonic Mass Completeness}

We must determine the stellar and baryonic mass completeness limits of the RESOLVE-B and ECO data sets to correctly interpret the SMF and BMF. We have already set the luminosity completeness limits based on where the $M_{r \text {,tot }}$ distribution of the data falls off (Figures 1(b) and 7). For ECO, the luminosity completeness limit was taken to be $-17.33 \mathrm{mag}$, the SDSS main redshift survey apparent magnitude limit as converted to our absolute magnitude system at our largest redshift, and we accounted for incompleteness in SDSS above its stated completeness limit by applying empirical completeness corrections based on RESOLVE-B (Section 3.6.2). For RESOLVE-B, the luminosity completeness limit was taken to be -17.0 mag without empirical completeness corrections, due to extended redshift coverage in Stripe 82. The lack of corrections implies that, over the range from -17.0 to -17.33 , RESOLVE-B underrepresents galaxy counts, as illustrated in Figure 1(b).

We can determine stellar and baryonic mass completeness limits by examining the scatter in stellar and baryonic mass near the luminosity completeness limits. In Figure 8(a), we plot stellar and baryonic mass as a function of $M_{r \text {,tot }}$ for RESOLVEB. We estimate mass completeness limits by finding the percentage of galaxies in RESOLVE-B with masses above a given mass limit that are fainter than our luminosity completeness limit for either RESOLVE-B or ECO (-17.0 and -17.33 , respectively). We require this percentage to be $<2 \%$. For ECO, the resulting stellar and baryonic mass limits are $\log \left(M_{\text {star }}\right)=8.9$ and $\log \left(M_{\text {bary }}\right)=9.4($ marked as thin red and blue dash-dotted lines in Figure 8(a)). The ECO mass completeness limits are independent of the completeness corrections computed for ECO. For RESOLVE-B, the stellar and baryonic mass limits are $\log \left(M_{\text {star }}\right)=8.7$ and $\log \left(M_{\text {bary }}\right)=9.1$ (marked as thick dashed red and blue lines). We note that the baryonic mass limits for RESOLVE-B and ECO extend to the high-mass dwarf regime, below the gasrichness threshold scale identified in $\mathrm{K} 13\left(M_{\text {bary }} \sim 10^{9.9} M_{\odot}\right)$.

Since RESOLVE-B is somewhat incomplete over the range from -17.0 to -17.33 , we check the robustness of our mass completeness limits by measuring the percentage of galaxies with masses above the aforementioned RESOLVE-B mass completeness limits $\left(M_{\text {star }}=10^{8.7} M_{\odot}\right.$ and $\left.M_{\text {bary }}=10^{9.1} M_{\odot}\right)$, but with $M_{r \text {,tot }}$ fainter than -17.33 rather than -17.0 . We find that the percentage of "missed" galaxies increases to $4 \%$ for RESOLVE-B with the higher luminosity limit. Since this increase is modest, we use the RESOLVE-B mass completeness limits determined at $M_{r \text {,tot }}=-17.0$ in the mass function analysis. However, we have tested our low-mass slope measurements in Section 5.2.2 with a stellar mass limit of $10^{8.9} M_{\odot}$ instead of $10^{8.7} M_{\odot}$, and the differences are small and well within the errors.

In Figure 8(b), we show the stellar and baryonic mass-tolight ratios as a function of $M_{r \text {,tot }}$ for RESOLVE-B. The mass completeness limits are converted to the limiting mass-to-light ratio at a given $M_{r \text {,tot }}$ and are shown as lines of constant mass corresponding to those in panel (a). The data sets are most complete for galaxies brighter than the luminosity completeness limit and having mass-to-light ratios brighter than the limiting ratios for a given $M_{r \text {,tot }}$.

\section{STATISTICAL ANALYSIS OF STELLAR AND BARYONIC MASS FUNCTIONS}

In this section, we describe our new "cross-bin sampling" method for measuring the SMF and BMF, taking into account the full stellar and baryonic mass likelihood distributions of galaxies. We use the full likelihood distributions of stellar and baryonic mass because the widths of these distributions are often much larger than the bin size used to construct the mass function $(\sim 0.1-0.2 \mathrm{dex})$. For the LF, this issue is of little concern because photometry errors are typically smaller than the bin sizes used to construct the LF, so uncertainties can be assigned to the LF using just the Poisson counting noise. In contrast, for the SMF and BMF, the uncertainty on the mass measurement itself may spill over several bins. Therefore we have devised the cross-bin sampling method that makes use of the full mass likelihood distributions to determine uncertainty bands around the derived mass functions. 

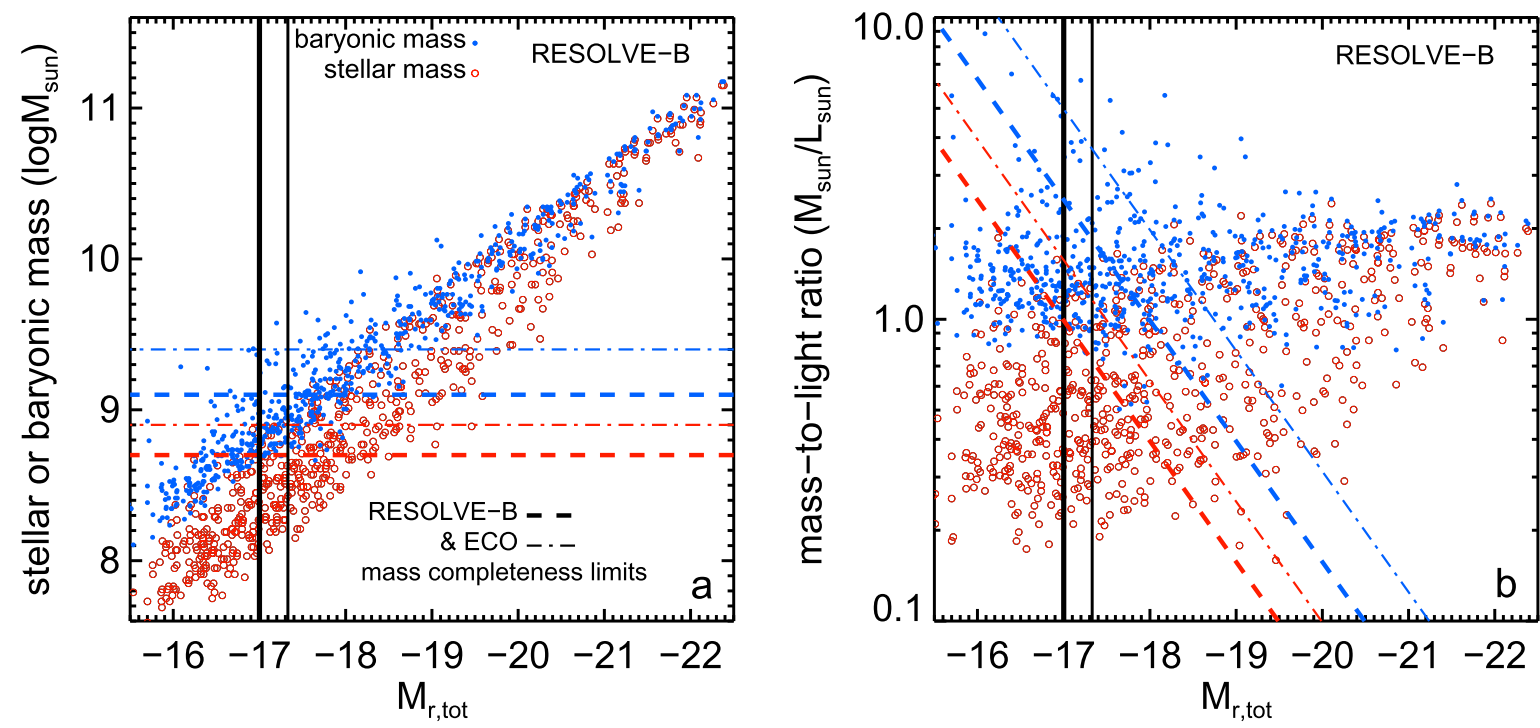

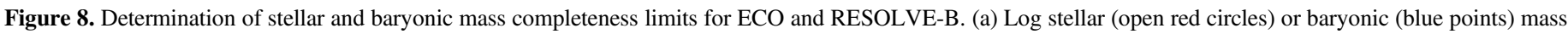

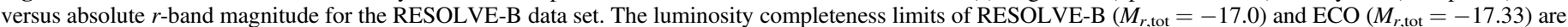

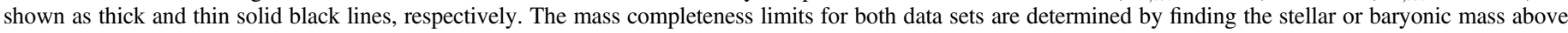

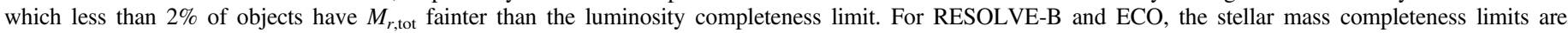

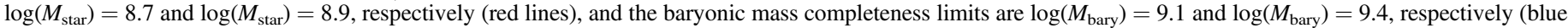

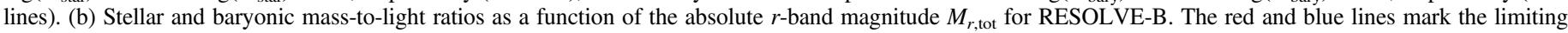

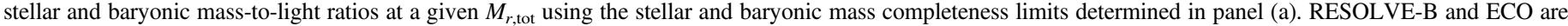

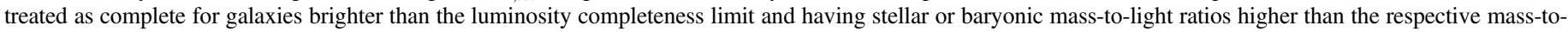
light ratio limits.

We first construct the normalized stellar or baryonic mass likelihood distributions for the entire RESOLVE-B and ECO data sets from the outputs of the SED fitting code (Section 3.2) and the baryonic mass calculations (Section 3.4.2). The stellar or baryonic mass likelihood distribution for each galaxy is binned in 0.01 dex intervals, much smaller than the typical mass distribution $1 \sigma$ widths of $\sim 0.25 \mathrm{dex}$. We then sum the likelihoods from all the galaxies at each small mass interval and normalize the entire distribution by the number of galaxies in the data set. As a simple example, if we started with a sample of 10 galaxies all with the same stellar mass likelihood distribution, this procedure would yield a normalized sample likelihood equal to the distribution for any one of the 10 galaxies.

To determine the SMF or BMF and the 68\% and $95 \%$ confidence intervals around that function for a given data set, we perform repeated Monte Carlo sampling of the corresponding sample stellar or baryonic mass likelihood distribution using the inverse transform sampling method. The inverse transform sampling method allows one to sample randomly from any probability distribution if its cumulative distribution function (cdf) is known. A number drawn from a uniform probability distribution between 0 and 1 (whose cdf is also a uniform probability distribution between 0 and 1) can then be used to look up the nonuniform distribution at the same integrated probability location within its cdf. To apply this method, we first cumulatively sum the sample stellar or baryonic mass likelihood distribution to produce the stellar or baryonic mass cdf. Second, we simulate a RESOLVE-B or ECO galaxy population by drawing $N$ values from a uniform probability distribution (0-1), where $N$ is drawn from a Poisson distribution with a mean value $\langle N>$ equal to the number of galaxies in the data set. Third, we use the inverse transform sampling method to look up the stellar or baryonic masses in the stellar or baryonic mass cdfs that correspond to the $N$ values just selected between 0 and 1, assigning a stellar or baryonic mass to each galaxy. Lastly, we bin the mass function into 0.2 dex bins. We perform this procedure 1000 times to create 1000 mass functions. From these 1000 stellar or baryonic mass functions, we determine the median and the $1 \sigma$ and $2 \sigma$ upper and lower bounds (16\%-84\% and 2.5\%-97.5\% percentile ranges) within each bin.

Figure 9 shows that our new SMF created through this crossbin sampling process (the $1 \sigma$ bounds shown in dark green and the $2 \sigma$ bounds shown in light green) has a shape similar to the traditional SMF, which uses the single-value stellar mass (median of each galaxy's stellar mass likelihood distribution) and assumes Poisson error bars in each bin. There are, however, noticeable deviations around $10^{10.5} M_{\odot}$. Using the traditional approach, we might overinterpret the dip/spike feature occurring at $\sim 10^{10.5} M_{\odot}$, which has been effectively smoothed over in the cross-bin sampling method, by taking into account the full likelihood distributions. Throughout the rest of this paper we will use $1 \sigma$ bounds to show the SMF and BMF uncertainty bands.

For ECO, we slightly modify this general methodology to include completeness corrections. Before constructing the normalized stellar or baryonic mass likelihood distribution for the ECO data set, we weight each galaxy's individual mass likelihood distribution by its completeness correction factor. The overall ECO mass likelihood distribution is the sum of these weighted distributions, and the "effective" total number of galaxies in the data set $\langle N\rangle$ is the total of all the completeness correction factors rather than the literal number of galaxies in the observed ECO data set. We run the Monte Carlo trials for the ECO data set 1000 times for the completeness corrections computed using $M_{r, \text { tot }}$ and $\mu_{r}$ and 1000 times for the completeness corrections computed using 


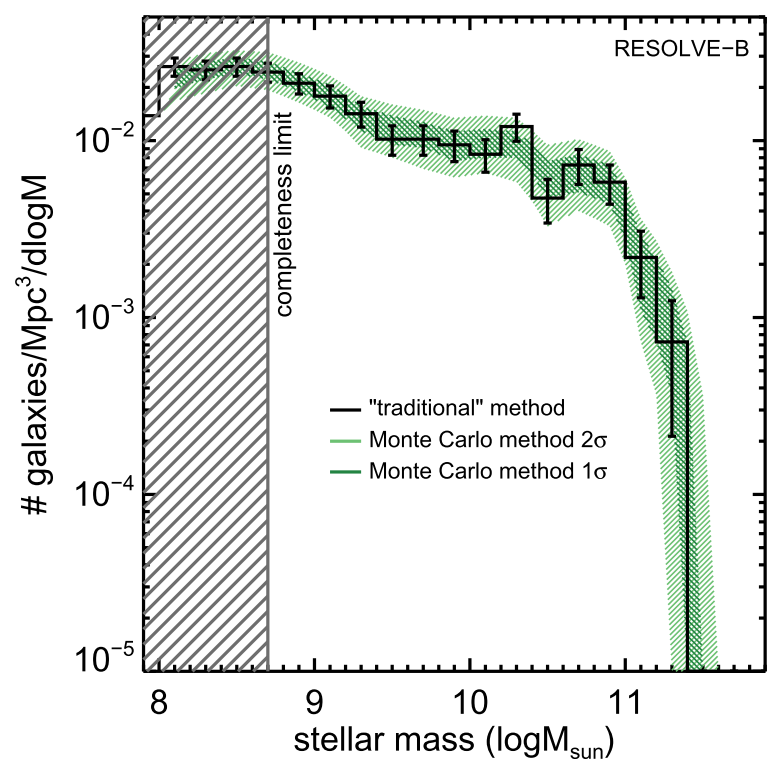

Figure 9. RESOLVE-B SMF calculated two different ways. The SMF is complete down to $10^{8.7} M_{\odot}$, marked by the dark gray line. The solid black histogram shows the number of galaxies per $d \log M \mathrm{Mpc}^{-3}$ when using the median stellar mass from each galaxy's stellar mass likelihood distribution (see Section 3.2) with Poisson error bars. The dark and light green shaded regions show the $68 \%$ and $95 \%$ confidence intervals for the SMF sampled from the stellar mass likelihood distribution for the entire galaxy data set, as described in Section 4. The Poisson error bars are similar in width to the $68 \%$ confidence intervals. The cross-bin sampling technique, however, yields a smoother SMF that takes into account the fact that stellar mass uncertainties can be much larger than the typical bin size of $\sim 0.2$ dex used to construct the SMF.

$M_{r, \text { tot }}$ and $(g-\mathrm{i})^{m}$. We compute the 16th, 50th, and 84th percentiles for the ECO mass functions based on the two completeness corrections. The ECO mass function is reported as the average between the two 50th percentile measurements in each stellar or baryonic mass bin. To estimate the uncertainty bands for the ECO mass functions, in each bin we choose the larger of the two 84th percentile values and the smaller of the two 16th percentile values determined for the two completeness-corrected mass functions.

\section{STELLAR AND BARYONIC MASS FUNCTIONS}

In this section, we examine the shape of the SMF and BMF for RESOLVE-B and ECO using the cross-bin sampling technique described in Section 4. First we check whether the features in the mass functions are robust to different stellar population models. We then compare our overall SMF and BMF with each other, with mass functions from the literature, and with the predicted HMF, paying special attention to the slope at the low-mass end. Lastly, we break down the SMF and BMF into "conditional mass functions" (mass functions divided into halo mass regimes and further by central/satellite designation) to analyze how the shape of the mass function depends on group halo mass.

We provide our raw SMFs and BMFs for RESOLVE-B and ECO in a machine-readable table, the columns of which are given in Table 2. For each mass function, we provide the median, 16th, and 84th percentiles. We only include values above the respective mass completeness limit for each data set.

\subsection{Choice of Stellar Population Models}

To check whether the shape of the SMF is dependent on the assumed grid of star-formation histories, we compare the SMFs resulting from the cross-bin sampling method for both model sets described in Section 3.2. The two SMFs are mostly similar, with a $\sim 0.08$ dex zero-point offset such that the model set $a$ SMF is shifted toward lower masses, similar to the $\sim 0.1$ dex offset previously reported in K13. Taking into account this shift, we find that the SMFs are extremely similar, except for the last two bins above the stellar mass completeness limit, where the $b$ model set is slightly steeper. At these masses, the dwarf galaxy stellar mass likelihood distributions are somewhat sensitive to modeling choices; however, the two model sets agree within their uncertainty bands after correcting for the zero-point offset. For simplicity, we use model set $a$ for the remainder of this work. This choice enables a direct comparison of the features in our mass functions with the threshold and bimodality mass scales described in K13. We note that our stellar masses are consistent with most others in the literature (see Section 3.2), apart from those in Bell et al. (2003b).

\subsection{Overall $S M F$ and $B M F$}

In this section, we examine the shape of the RESOLVE-B and ECO SMF and BMF, which are shown in Figures 10 and 11. We examine the results of single and double Schechter fitting and compare with previous mass functions from the literature. We also directly compare the SMF and BMF with each other and with the theoretical HMF in Figure 12.

\subsubsection{Mass Function Fit Parameters}

We use the Markov chain Monte Carlo ensemble sampler emcee (Foreman-Mackey et al. 2013) to fit both Schechter and double Schechter functions to the RESOLVE-B and ECO mass functions in a Bayesian framework. The single Schechter function follows the traditional form as a function of galaxy mass or $m$ :

$$
\phi(m) d \log (m)=\phi_{*}\left(\frac{m}{M_{*}}\right)^{(\alpha+1)} \exp \left(\frac{-m}{M_{*}}\right) d \log (m) .
$$

Here $M_{*}$ is the characteristic mass scale, $\alpha$ is the power-law rise at the low-mass end, and $\phi_{*}$ is the overall normalization at $M_{*}$.

We also fit a double Schechter function that allows for two low-mass power-law slopes and two normalization parameters, following the form described in Baldry et al. (2008):

$$
\begin{aligned}
\phi(m) d \log (m)= & \exp \left(\frac{-m}{M_{*}}\right) d \log (m) \\
& \times\left[\phi_{* 1}\left(\frac{m}{M_{*}}\right)^{\left(\alpha_{1}+1\right)}+\phi_{* 2}\left(\frac{m}{M_{*}}\right)^{\left(\alpha_{2}+1\right)}\right] .
\end{aligned}
$$

For our Bayesian parameter estimation, we assume uniform priors on all single Schechter function parameters over ranges encompassing previous estimates of all the values. We also assume uniform priors on all double Schechter function parameters with the additional requirement that $\alpha_{2}$ be less than $\alpha_{1}$ (i.e., $\alpha_{2}$ must have a steeper power-law slope than $\alpha_{1}$ ), similar to Baldry et al. (2008). 
Table 2

All RESOLVE-B and ECO Stellar and Baryonic Mass Functions

\begin{tabular}{|c|c|}
\hline $\begin{array}{l}\text { Column } \\
\text { Number }\end{array}$ & $\begin{array}{l}\text { Mass Function Description } \\
\left(\phi_{16}, \phi_{\text {med }}, \phi_{84}\right)\end{array}$ \\
\hline 1 & stellar or baryonic mass \\
\hline $2-4$ & RESOLVE-B SMF \\
\hline $5-7$ & RESOLVE-B SMF $\log \left(M_{\text {halo }}\right)<11.4$ \\
\hline $8-10$ & RESOLVE-B SMF $11.4<\log \left(M_{\text {halo }}\right)<12.0$ \\
\hline $11-13$ & RESOLVE-B SMF $12.0<\log \left(M_{\text {halo }}\right)<13.5$ \\
\hline $14-16$ & RESOLVE-B SMF central $\log \left(M_{\text {halo }}\right)<11.4$ \\
\hline $17-19$ & RESOLVE-B SMF central $11.4<\log \left(M_{\text {halo }}\right)<12.0$ \\
\hline $20-22$ & RESOLVE-B SMF central $12.0<\log \left(M_{\text {halo }}\right)<13.5$ \\
\hline $23-25$ & RESOLVE-B SMF satellite $11.4<\log \left(M_{\text {halo }}\right)<12.0$ \\
\hline $26-28$ & RESOLVE-B SMF satellite $12.0<\log \left(M_{\text {halo }}\right)<13.5$ \\
\hline 29-31 & RESOLVE-B BMF \\
\hline $32-34$ & RESOLVE-B BMF $\log \left(M_{\text {halo }}\right)<11.4$ \\
\hline $35-37$ & RESOLVE-B BMF $11.4<\log \left(M_{\text {halo }}\right)<12.0$ \\
\hline $38-40$ & RESOLVE-B BMF $12.0<\log \left(M_{\text {halo }}\right)<13.5$ \\
\hline $41-43$ & RESOLVE-B BMF central $\log \left(M_{\text {halo }}\right)<11.4$ \\
\hline $44-46$ & RESOLVE-B BMF central $11.4<\log \left(M_{\text {halo }}\right)<12.0$ \\
\hline $47-49$ & RESOLVE-B BMF central $12.0<\log \left(M_{\text {halo }}\right)<13.5$ \\
\hline $50-52$ & RESOLVE-B BMF satellite $11.4<\log \left(M_{\text {halo }}\right)<12.0$ \\
\hline $53-55$ & RESOLVE-B BMF satellite $12.0<\log \left(M_{\text {halo }}\right)<13.5$ \\
\hline $56-58$ & ECO SMF \\
\hline $59-61$ & ECO SMF $\log \left(M_{\text {halo }}\right)<11.4$ \\
\hline $62-64$ & ECO SMF $11.4<\log \left(M_{\text {halo }}\right)<12.0$ \\
\hline $65-67$ & ECO SMF $12.0<\log \left(M_{\text {halo }}\right)<13.5$ \\
\hline $68-70$ & ECO SMF $\log \left(M_{\text {halo }}\right)>13.5$ \\
\hline $71-73$ & ECO SMF central $\log \left(M_{\text {halo }}\right)<11.4$ \\
\hline $74-76$ & ECO SMF central $11.4<\log \left(M_{\text {halo }}\right)<12.0$ \\
\hline 77-79 & ECO SMF central $12.0<\log \left(M_{\text {halo }}\right)<13.5$ \\
\hline $80-82$ & ECO SMF central $\log \left(M_{\text {halo }}\right)>13.5$ \\
\hline $83-85$ & ECO SMF satellite $11.4<\log \left(M_{\text {halo }}\right)<12.0$ \\
\hline $86-88$ & ECO SMF satellite $12.0<\log \left(M_{\text {halo }}\right)<13.5$ \\
\hline 89-91 & ECO SMF satellite $\log \left(M_{\text {halo }}\right)>13.5$ \\
\hline $92-94$ & ECO BMF \\
\hline 95-97 & ECO BMF $\log \left(M_{\text {halo }}\right)<11.4$ \\
\hline $98-100$ & ECO BMF $11.4<\log \left(M_{\text {halo }}\right)<12.0$ \\
\hline $101-103$ & ECO BMF $12.0<\log \left(M_{\text {halo }}\right)<13.5$ \\
\hline 104-106 & ECO BMF $\log \left(M_{\text {halo }}\right)>13.5$ \\
\hline 107-109 & ECO BMF central $\log \left(M_{\text {halo }}\right)<11.4$ \\
\hline $110-112$ & ECO BMF central $11.4<\log \left(M_{\text {halo }}\right)<12.0$ \\
\hline $113-115$ & ECO BMF central $12.0<\log \left(M_{\text {halo }}\right)<13.5$ \\
\hline $116-118$ & ECO BMF central $\log \left(M_{\text {halo }}\right)>13.5$ \\
\hline $119-121$ & ECO BMF satellite $11.4<\log \left(M_{\text {halo }}\right)<12.0$ \\
\hline $122-124$ & ECO BMF satellite $12.0<\log \left(M_{\text {halo }}\right)<13.5$ \\
\hline $125-127$ & ECO BMF satellite $\log \left(M_{\text {halo }}\right)>13.5$ \\
\hline
\end{tabular}

Note. The first column has units of $\log M_{\text {Sun }}$, and all other columns have units of $d \log M^{-1} \mathrm{Mpc}^{-3}$. The three columns for each mass function represent the 16th, 50th, and 84th percentiles.

(This table is available in machine-readable form.)

The emcee code uses a Markov chain Monte Carlo ensemble sampler to fill out the parameter space. For the single Schechter function, we use 100 walkers over 100 steps after a burn-in of 100 steps. For the double Schechter function, we use 400 walkers over 100 steps after a burn-in of 400 steps. To assess the convergence of the chains, we measured the autocorrelation time for each parameter's chain and set the burn-in number of steps to be a few times the autocorrelation time (per the guidelines discussed in Foreman-Mackey et al. 2013). We also visually inspected the chains to ensure that they properly sampled the parameter space, and we calculated the mean acceptance fraction of the chains to be $\sim 0.5$ and $\sim 0.35$ for the single and double Schechter fits, respectively (within the acceptable range $0.2-0.5$, as discussed in Foreman-Mackey et al. 2013). We report the median of the marginalized posterior probability distributions for each parameter in Table 3 with error bars showing the 16th and 84th percentiles.

In our parameter fitting, we do not consider any error term that is due to cosmic variance, i.e., related to the halo mass mix or overall density within our data sets, as has been done in some previous work (e.g., Leauthaud et al. 2011; Smith 2012). Using mock catalogs, these works have shown that individual bins within the SMF or LF are correlated with each other for any given data set, reflecting its overall environmental density and mix of environments and the fact that a few high-mass halos contribute most of the galaxies at the bright end. Smith (2012) finds that inclusion of the full covariance matrix yields Schechter parameter fits differing by up to $2 \sigma$ compared to fits using only Poisson errors. The parameters most affected are $\alpha$, which becomes steeper, and $L_{*}$, which becomes fainter, after taking into account these covariances. However, since we will show in Section 5.3 that the mass function is not universal but depends on halo mass, our approach does not treat environmental variance (as defined by the group halo mass distribution) as an "error" but rather as a physical manifestation of the fact that the mass function varies with the group halo mass distribution in predictable ways. As a result, the variation in Schechter fit parameters between RESOLVE-B and ECO may be (unsurprisingly) larger than our quoted errors because of their different group halo mass distributions.

\subsubsection{The $S M F$}

The RESOLVE-B and ECO SMFs drop off steeply for masses $\gtrsim 10^{10.8} M_{\odot}$, which is near the "knee" of the Schechter function that joins the steep exponential falloff toward highermass galaxies and the power-law rise toward lower-mass galaxies. Based on our single and double Schechter function fits, we find that the knee occurs at $\sim 10^{10.8-11.1} M_{\odot}$ for both data sets. The knee of the SMF has been measured to be $\sim 10^{10.7} M_{\odot}$ in previous works, including Panter et al. (2007), Baldry et al. (2008, 2012), and Peng et al. (2010).

We note, however, that at high masses neither the single nor double Schechter functions fit our SMFs well, indicating that the exponential falloff at high masses is not a good model for our two data sets. For RESOLVE-B, the falloff is steeper than the fits, while for ECO the falloff is shallower. The shallowness of the ECO falloff may be in line with the results from Bernardi et al. (2013), who recovered more light from bright galaxies using PyMorph and found a shallower falloff in the LF. RESOLVE-B, on the other hand, has relatively few extremely bright galaxies since it has no large clusters with mass $>10^{13.5} M_{\odot}$, potentially leading to the steeper falloff. We also note that the GAMA-derived SMF from Baldry et al. (2012) has a falloff between those of RESOLVE-B and ECO. While GAMA covers a relatively small volume, they still have more massive clusters than found in RESOLVE-B (Robotham et al. 2011).

Below $\sim 10^{10.8} M_{\odot}$, the RESOLVE-B and ECO SMFs rise toward lower masses. However, the slope plateaus over a mass range of $10^{9.5-10.2} M_{\odot}$. The plateau feature in the SMF has been observed in several previous studies (Baldry et al. 2008; Drory et al. 2009; Li \& White 2009; Peng et al. 2010), motivating the use of the double Schechter function to fit the low-mass end of the SMF. Single Schechter functions, such as the dashed black line from Panter et al. (2007) in Figure 10(a) and the dark green 


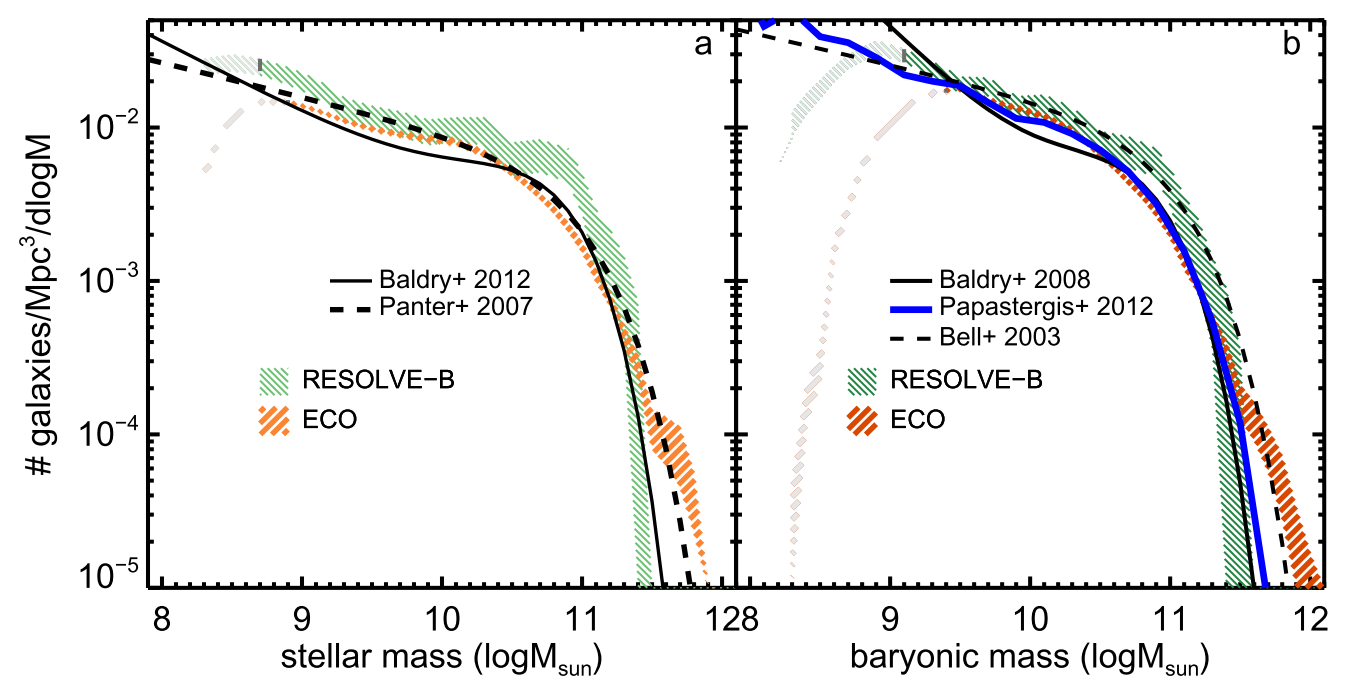

Figure 10. RESOLVE-B and ECO SMFs and BMFs using the cross-bin sampling technique with comparison to previous work. The uncertainties due to cosmic variance are not included in the error budgets of the mass functions. (a) SMFs for RESOLVE-B (light green) and ECO (light orange), with shaded regions showing the 16-84th percentile confidence intervals. Incomplete regions of the mass functions are shaded lighter. The black solid line shows the double Schechter function fit from Baldry et al. (2012), and the dashed line shows the single Schechter function fit from Panter et al. (2007) (Section 5.2.2). (b) BMFs for RESOLVE-B (dark green) and ECO (dark orange). The solid blue line comes from the measured BMF from Papastergis et al. (2012) using a combination of SDSS and ALFALFA. The solid black line shows the inferred BMF from Baldry et al. (2008), and the dashed line is from Bell et al. (2003a) (Section 5.2.3).

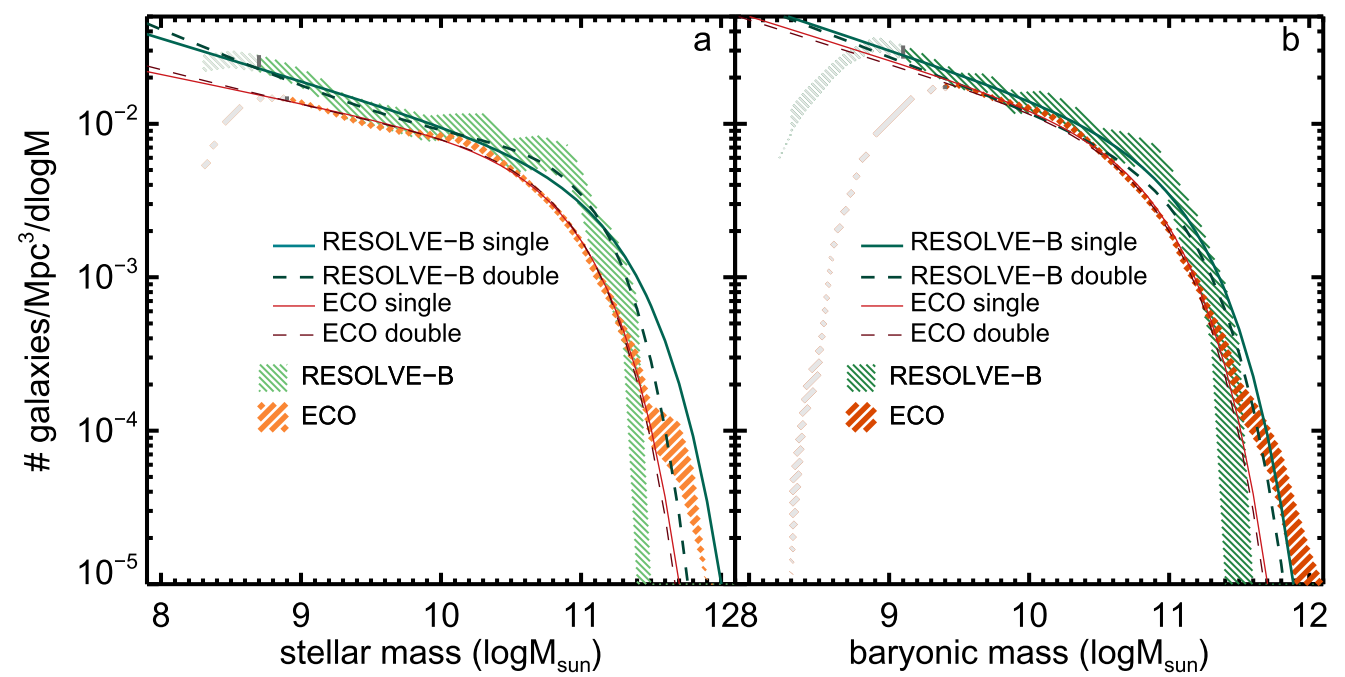

Figure 11. RESOLVE-B and ECO SMFs and BMFs using the cross-bin sampling technique with single and double Schechter function fits. The uncertainties due to cosmic variance are not included in the error budgets of the mass functions. (a) SMFs for RESOLVE-B (light green) and ECO (light orange), with shaded regions showing the 16-84th percentile confidence intervals. Incomplete regions of the mass functions are shaded lighter. The solid green and orange lines show single Schechter function fits to RESOLVE-B and ECO, respectively, and appear to be inadequate fits to the data. The dashed green and orange lines show the double Schechter function fits to RESOLVE-B and ECO, respectively. These fits, however, are not much improved over the single Schechter function fits. (b) BMFs for RESOLVE-B (dark green) and ECO (dark orange). Again, the solid and dashed green and orange lines show the single and double Schechter function fits for RESOLVE-B and ECO, respectively. The additional parameters of the double Schechter function are unnecessary for fitting the shape of the BMF.

and orange solid lines based on our fits in Figure 11(a), cannot reproduce the shape of the SMFs. To model the plateau, others have implemented double (Baldry et al. 2008, 2012; Drory et al. 2009; Peng et al. 2010) and even triple (Li \& White 2009) Schechter functions. We observe this plateau feature in the SMF (seen more easily in Figure 12 with reference to the BMF and without overlapping fit lines), but we find that the double Schechter function does not yield a much better fit than the single Schechter function for either the RESOLVE-B or ECO SMFs. Therefore, to measure the plateau slope and the lowmass slope, we fit lines to the SMF over a range of $\log \left(M_{\text {star }}\right)=9.5-10.1$ for the plateau and $\log \left(M_{\text {star }}\right) \leqslant 9.5$ for the low-mass end. These values have been determined by examining where the plateau and steep low-mass upturn features occur in Figure 11. With this technique, we measure a relatively flat slope just below the mass function knee $\left(\alpha_{\text {plateau }}=-1.14 \pm 0.18\right.$ and $-1.14 \pm 0.05$ for RESOLVE-B and $\mathrm{ECO}$ ) and a more steeply rising slope below the gasrichness threshold mass $\left(\alpha_{\text {low-mass }}=-1.44 \pm 0.11\right.$ and $-1.30 \pm 0.04$ for RESOLVE-B and ECO), albeit at $\sim 1-2 \sigma$ significance. For comparison, Baldry et al. (2012) find shallow and steep slopes of $\alpha_{1}=-0.35$ and $\alpha_{2}=-1.47$ in their double Schechter function fit.

Comparing the normalizations of RESOLVE-B and ECO in the double Schechter fits, we see that, for high masses, $\phi_{*, 1}$ is much lower for ECO than for RESOLVE-B (0.0034 versus 


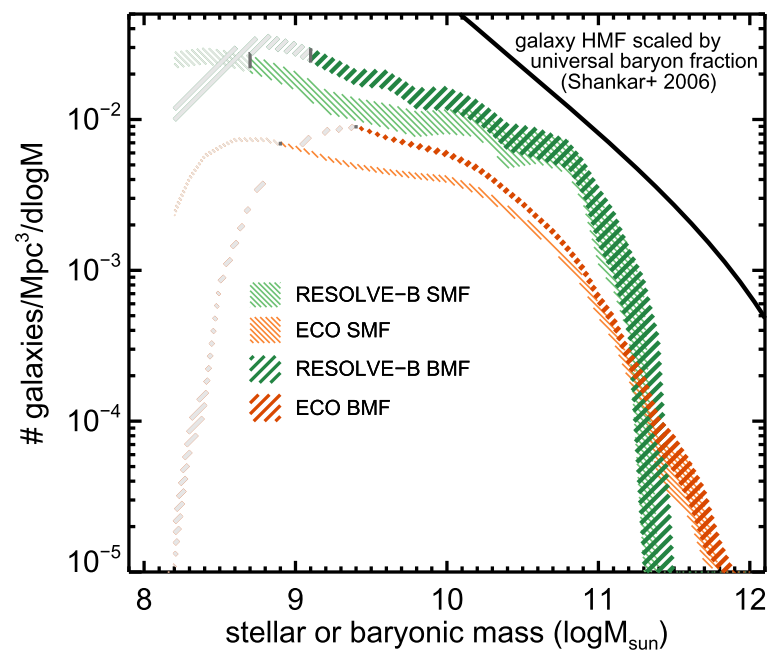

Figure 12. Direct comparison of the RESOLVE-B and ECO SMF and BMF using the cross-bin sampling technique. The SMF and BMF are plotted over each other for both data sets using the same color scheme as in Figure 10. The ECO mass functions have been scaled down by a factor of two for clarity, and the incomplete regions are shaded gray. For both data sets, the BMF diverges from the SMF, beginning near the bimodality mass of $M_{\text {bary }} \sim 10^{10.5} M_{\odot}$ and becoming significant below the gas-richness threshold mass of $M_{\text {bary }} \sim 10^{9.9} M_{\odot}$. The galaxy HMF (including the contribution from subhalos) from Shankar et al. (2006) is shown scaled by the universal baryon fraction, 0.15 , for comparison.

0.0090), while $\phi_{*, 2}$ is much more similar. The lower normalization of ECO is not unexpected, due to the overall smaller number density in the ECO catalog, as described in Section 2.4. Variations in number density with sample size (i.e., cosmic variance) will affect the overall normalization, as seen by the vertical displacement of several previous works in Figure 10.

\subsubsection{The $B M F$}

The RESOLVE-B and ECO BMFs also exhibit a steep dropoff for masses $>10^{10.8} M_{\odot}$, which is consistent with previous work (Bell et al. 2003a; Baldry et al. 2008; Papastergis et al. 2012), although the drop-off occurs at higher masses in Bell et al. (2003a), due to the different mass scales used.

At masses below $10^{10.8} M_{\odot}$, however, the BMFs rise as a straight power law toward lower masses. The BMF shape is actually better described by the single Schechter function shape than is the SMF. The double Schechter function fits given in Table 3 do not significantly improve on the single Schechter function fits (see Figure 11(b)). The low-mass slope given by the single Schechter function fits for RESOLVE-B and ECO is $\alpha \sim-1.3$, steeper than the low-mass slope of the SMF just below the knee $\left(\alpha_{\text {plateau }} \sim-1.14\right)$. We find a low-mass slope that is slightly steeper than the slope determined in Bell et al. (2003a), which used stellar masses inconsistent with our own and a different $\mathrm{HI}$ mass estimation technique.

Comparing with the observed BMF from Papastergis et al. (2012), which is shown as a blue solid line in Figure 10(b) and uses ALFALFA HI measurements to construct the BMF, we find that it is mostly consistent with the ECO BMF, except at the largest masses, where the number density of galaxies is small. Neither the RESOLVE-B nor ECO BMFs are fit well by the inferred BMF from Baldry et al. (2008), which is based on the SMF and infers baryonic mass via the stellar mass- metallicity relation and other scaling relations between metallicity and stellar mass fraction (solid black line). This inferred BMF is constructed to include all baryonic components (including stars and cold and warm gas), although we note that it follows the shape of the SMF, from which it is derived, until rising steeply below $M_{\text {bary }}<10^{9.7} M_{\odot}$.

\subsubsection{Divergence of $S M F$ and $B M F$}

To directly compare the RESOLVE-B and ECO SMFs and BMFs, we plot all of the mass functions in Figure 12. The ECO mass functions have been scaled down for clarity. We note that the relative difference between the SMF and BMF are similar for both RESOLVE and ECO, with the BMF $\sim 0.17$ dex higher than the SMF at $10^{10} M_{\odot}$, rising to $\sim 0.25$ dex higher than the SMF at $10^{9.5} M_{\odot}$.

It is apparent that the SMF and BMF are effectively the same at large masses, thus dropping off at a similar knee of $M_{*} \sim 10^{10.8} M_{\odot}$. This result is not surprising since the drop-off mass scale is above the bimodality mass scale of $\sim 10^{10.5} M_{\odot}$ identified in Kauffmann et al. (2003b), above which galaxies tend to be bulge-dominated, red, and quenched of star formation with little to no cold gas (K13).

Going down across the bimodality mass $\left(\sim 10^{10.5} M_{\odot}\right)$, we find that the BMF and SMF start to diverge by $>0.1$ dex (albeit within the error bars for RESOLVE-B). The SMF plateaus while the BMF rises as a straight power law. For RESOLVE-B, the divergence between the two mass functions becomes significant below a stellar or baryonic mass of $10^{9.7-9.9} M_{\odot}$, the gas-richness threshold scale identified in K13. Below the gasrichness threshold mass emerges a significant population of galaxies that may have as much or more gas than stellar mass. These gas-rich galaxies fill in the plateau region and push the $\mathrm{BMF}$ to rise as a straight power law.

At the lowest galaxy masses $\left(M_{\text {star }}<10^{9.5} M_{\odot}\right)$, we see that the SMF starts to rise more steeply. We cannot determine whether the BMF follows suit, as our RESOLVE-B and ECO data sets are limited at $M_{\text {bary }} \sim 10^{9.1} M_{\odot}$ and $10^{9.4} M_{\odot}$, respectively.

Although the SMF and BMF at high masses are similar when comparing within each data set, there are large differences between the mass functions between the RESOLVE-B and ECO data sets. First, as can be noted in Figures 10 and 11, the RESOLVE-B mass functions are elevated over the ECO mass functions. This relates to the overall higher density of RESOLVE-B, due to cosmic variance (as described in Section 2.4). Second, while the ECO SMF and BMF decline gradually, the RESOLVE-B stellar and BMFs drop off much more abruptly. The abruptness of the turnover in RESOLVE-B gives the appearance of a "bump" at the high-mass end of the SMF and BMF. We explore these differences and tie them to the different group halo mass distributions sampled within ECO and RESOLVE-B in Section 5.3.

\subsubsection{Relationship of Observed Galaxy to Theoretical HMFs}

Finally, we compare the RESOLVE-B and ECO SMFs and BMFs to the galaxy HMF derived in Shankar et al. (2006), which includes the contribution from subhalos and removes the subhalo contribution to large group halos. The galaxy HMF, which has been scaled by the universal baryon fraction of $\sim 0.15$ (Planck Collaboration et al. 2014) to enable direct comparison to galaxy masses, has a steep slope with 
Table 3

Single and Double Schechter Function Parameters for RESOLVE-B and ECO Stellar and Baryonic Mass Functions

\begin{tabular}{|c|c|c|c|c|c|}
\hline Mass Function & $\begin{array}{l}\log \left(M_{*}\right) \\
\log \left(M_{\odot}\right)\end{array}$ & $10^{3} \times\left(\operatorname{Mpc}^{\phi_{* 1}} d \log M\right)^{-1}$ & $\alpha_{1}$ & $10^{3} \times\left(\mathrm{Mpc}^{3} d \log M\right)^{-1}$ & $\alpha_{2}$ \\
\hline RESOLVE-B SMF & $11.25_{-0.19}^{+0.25}$ & $4.47_{-1.53}^{+1.82}$ & $-1.28_{-0.05}^{+0.06}$ & $\ldots$ & $\ldots$ \\
\hline RESOLVE-B SMF & $10.87_{-0.27}^{+0.33}$ & $9.00_{-8.47}^{+6.36}$ & $-0.52_{-0.49}^{+0.87}$ & $3.25_{-2.81}^{+3.00}$ & $-1.38_{-0.35}^{+0.13}$ \\
\hline ECO SMF & $10.92_{-0.03}^{+0.03}$ & $5.95_{-0.42}^{+0.41}$ & $-1.19_{-0.02}^{+0.02}$ & $\ldots$ & $\ldots$ \\
\hline ECO SMF & $10.87_{-0.06}^{+0.05}$ & $3.44_{-1.93}^{+2.25}$ & $-0.91_{-0.15}^{+0.23}$ & $3.62_{-1.78}^{+1.49}$ & $-1.26_{-0.11}^{+0.06}$ \\
\hline RESOLVE-B BMF & $11.11_{-0.16}^{+0.19}$ & $6.93_{-2.33}^{+2.56}$ & $-1.30_{-0.07}^{+0.06}$ & $\ldots$ & $\ldots$ \\
\hline RESOLVE-B BMF & $10.98_{-0.25}^{+0.22}$ & $2.74_{-2.66}^{+10.0}$ & $-0.48_{-0.64}^{+1.80}$ & $5.54_{-4.36}^{+3.86}$ & $-1.35_{-0.28}^{+0.10}$ \\
\hline ECO BMF & $10.92_{-0.04}^{+0.03}$ & $7.48_{-0.79}^{+0.85}$ & $-1.28_{-0.03}^{+0.03}$ & $\ldots$ & $\ldots$ \\
\hline ECO BMF & $10.89_{-0.07}^{+0.05}$ & $1.55_{-1.17}^{+2.70}$ & $-1.02_{-0.21}^{+1.69}$ & $6.27_{-2.54}^{+2.40}$ & $-1.30_{-0.06}^{+0.05}$ \\
\hline
\end{tabular}

$\alpha_{\mathrm{HMF}}=-1.84$ and is shown as a thick solid line in Figure 12. Although the low-mass slope of the BMF is steeper than that of the SMF, it is not nearly as steep as the galaxy HMF slope. We discuss this result in more detail in Section 6.

\subsection{Conditional SMF and BMF}

While there are many similarities between the RESOLVE-B and ECO SMF and BMF, Figures 10 and 11 reveal a significant difference in their shapes at high stellar/baryonic mass. One possible explanation is the different group halo mass distributions, since the volumes of both surveys are too small to escape cosmic variance. For example, RESOLVE-B, unlike ECO, has no high-mass clusters $>10^{13.5} M_{\odot}$. It does, however, have an overabundance of clusters of mass $\sim 10^{12.5} M_{\odot}$ and $10^{13.5} M_{\odot}$ compared to ECO (see Figure 5).

In this section we investigate how the shape of the mass function depends on group halo mass. We first define physically motivated halo mass regimes, then break down the mass functions within each halo mass regime. We then further break down the mass functions in each group halo mass regime into the central and satellite components. Finally, we analyze whether the high-mass discrepancy between RESOLVE-B and ECO can be explained by distinct group halo mass distributions.

To ensure that group finding and in particular that the choice of linking lengths described in Section 3.5.1 do not drive our results, we have performed the following analysis with the linking lengths of Berlind et al. (2006) (see Section 3.5.1). We do not find any significant differences with the results presented in this section using these alternate linking lengths.

\subsubsection{Definition of Group Halo Mass Regimes}

To examine the SMF and BMF in different group halo mass regimes, we use the group identifications and masses described in Section 3.5 to divide the RESOLVE-B and ECO data sets into four group halo mass regimes. These group halo mass regimes are (1) $M_{\text {halo }}<10^{11.4} M_{\odot}$, the "low-mass group" regime; (2) $M_{\text {halo }}$ between $10^{11.4} M_{\odot}$ and $10^{12.0} M_{\odot}$, the "intermediate-mass group" regime; (3) $M_{\text {halo }}$ between $10^{12.0} M_{\odot}$ and $10^{13.5} M_{\odot}$, the "large group" regime; and (4) $M_{\text {halo }}>10^{13.5} M_{\odot}$, the "cluster" regime, which applies only to ECO.

The low-mass group regime includes all group halos below $M_{\text {halo }}=10^{11.4} M_{\odot}$, which is the group halo mass that roughly corresponds to the gas-richness threshold mass identified in K13 as $10^{9.7} M_{\odot}$ in stellar mass and $10^{9.9} M_{\odot}$ in baryonic mass (see Figure 5(b)). Galaxies in halos with masses $<10^{11.4} M_{\odot}$ are generally low-mass central galaxies of mass comparable to the Large Magellanic Cloud (Kim et al. 1998) and have significant amounts of gas, resulting in a large increase in their baryonic masses compared to their stellar masses. It should be noted that we have not included a lower halo mass floor in defining this regime and that the lowest included extrapolated halo mass is $\sim 10^{10.5} M_{\odot}$. Such low-mass halos mostly indicate low-mass galaxies living in halos by themselves, at least down to our sample limits.

The intermediate-mass regime is in the range $M_{\text {halo }}=$ $10^{11.4}-10^{12.0} M_{\odot}$, which roughly corresponds to the central galaxy bimodality mass $\sim 10^{10.5} / 10^{10.6} M_{\odot}$ in stellar and baryonic mass (Kauffmann et al. 2003b and also K13). In this regime, we find nascent groups with only a few members (see Figures 5 and 8 from M15). Above group halo mass of $\sim 10^{12.0} M_{\odot}$ marks a transition in the central galaxy mass to halo mass relationship, where for large groups growth of the integrated galaxy mass in the halo becomes more dependent on the satellite inventory than on central mass growth (Conroy \& Wechsler 2009; Behroozi et al. 2010, 2013; Leauthaud et al. 2011, 2012). As shown in Figure 5(b), for nascent groups with mass $<10^{12.0} M_{\odot}$, central galaxies still have appreciable amounts of gas with a minimal satellite population. For large group halos with mass $>10^{12.0} M_{\odot}$, the halos start filling up with satellites, and the cold gas becomes less and less important to the overall baryonic mass of the central.

Our last group halo mass division is placed at $M_{\text {halo }}=10^{13.5} M_{\odot}$ between the large group and cluster regimes. While groups less massive than $10^{13.5} M_{\odot}$ tend to live in a range of large-scale structure overdensities, clusters above this halo mass division reside in the most dense structures (Carollo et al. 2013). Additionally, the group LF characteristic mass and faint-end slope values converge for groups more massive than $10^{13.5} M_{\odot}$ (Robotham et al. 2006). In RESOLVE$\mathrm{B}$, there are no halos more massive than $10^{13.5} M_{\odot}$, so this cluster regime only applies to ECO, which includes the Coma cluster.

\subsubsection{SMF and BMF by Group Halo Mass ("Conditional Mass Functions")}

Breaking down the RESOLVE-B and ECO mass functions into these four group halo mass regimes, shown in Figure 13, reveals complex structure within the overall galaxy mass functions. For instance, in increasingly higher halo mass regimes, we observe high-mass drop-offs that occur at higher galaxy mass. These drop-offs mark the natural boundary in the largest central galaxy mass for a given halo mass, as seen in Figure 5(b).

In both the RESOLVE-B and ECO intermediate (green) and large (orange) group halo mass regimes, we observe a peak in 
the SMF and BMF. For intermediate group halos, this peak occurs at $\sim 10^{10.2} M_{\odot}$, right between the gas-richness threshold and bimodality mass scales. For large group halos, the peak occurs at $\sim 10^{10.8} M_{\odot}$, above the bimodality mass scale and also near the knee of the overall mass functions. The peak in the large group halo regime appears to be causing the pronounced "bump" seen in the overall RESOLVE-B SMF and BMF. While the ECO data set also has this characteristic peak in its large group halo regime mass functions, the more gradual decline in the overall SMF and BMF for large galaxy masses seems to be due to the cluster galaxy population in ECO. The cluster population makes up $17 \%$ of galaxies with $M_{\text {star }}>10^{10.5} M_{\odot}$ and $31 \%$ of galaxies with $M_{\text {star }}>10^{11} M_{\odot}$ for ECO. The RESOLVE-B "bump," meanwhile, may be emphasized by the lack of a cluster galaxy population and the overabundance of halos in this large group halo regime, as shown in Figure 5(a).

It is clear that the low-mass slopes of the SMF and BMF in these different halo mass regimes are very different. In the cluster halo mass regime, the rise is quite smooth, although the slope appears to flatten out at lower masses, at least in part due to incompleteness in galaxy counts around the clusters caused by high fiber collision rates and by missing ultradiffuse galaxies like those recently found in the Coma and Virgo clusters (Mihos et al. 2015; van Dokkum et al. 2015). We note that Yamanoi et al. (2012) found a fairly flat slope for the Coma cluster LF down to galaxy magnitudes of $M_{R}=-14.0$, with a steep upturn for galaxies below our luminosity limit. Also, not all previous studies of cluster LFs have found steep slopes, and many cluster LF studies have relied on using statistical counts to remove background galaxies (e.g., Dressler 1978 and Goto et al. 2002).

In the intermediate and large group halo mass regimes, both RESOLVE-B and ECO have an intriguing falloff in galaxy number density for galaxy masses below the peak. While the intermediate group halo mass regime does not show evidence for a low-mass upturn, the RESOLVE-B large group halo mass regime does have a steeply rising low-mass slope. This steeply rising slope is not as apparent in ECO, but it is shallower. To examine this discrepancy further, we show a version of RESOLVE-B limited to $M_{r \text {,tot }}<-17.33$ to be consistent with ECO in panels (c) and (d) of Figure 13, and we find that the low-mass slope in the large group halo mass regime appears less steep but is still elevated above the intermediate halo mass regime mass functions. We posit that the difference is due to the overabundance of such large group mass halos in ECO and investigate further in Section 5.3.6. Finally, the low group halo regime mass functions show a steeply rising slope toward low galaxy masses. This breakdown shows that the low-mass slope of the BMF across different group halo mass regimes is not invariant. This result argues against the conjecture from Bell et al. (2003a) that the cluster and field BMFs might have similar low-mass slopes.

Finally, Figure 13 reflects the fact that low-mass galaxies in RESOLVE-B and ECO live in low-mass, mainly isolated group halos more often than in larger group mass halos. The crossover mass scales $\left(M_{\text {star }}<10^{9.5} M_{\odot}\right.$ and $\left.M_{\text {bary }}<10^{9.8} M_{\odot}\right)$ roughly correspond with the gas-richness threshold mass from K13. Thus, in the "high-mass dwarf" regime we probe, most dwarfs below the threshold mass live in low-mass halos rather than as members of clusters and large groups.

\subsubsection{Central Galaxy Mass Functions in Each Group Halo Mass Bin}

Next we examine how the central galaxy population in each group halo mass regime affects the shape of the SMF and $\mathrm{BMF}$ by breaking up the conditional mass functions according to central or satellite designation for RESOLVE-B and ECO in Figures 14 and 15, respectively.

The central galaxy SMFs and BMFs (center panels of Figures 14 and 15) appear in discrete, narrow "humps" whose peak mass value increases with increasing group halo mass. The centers of these humps correspond with the peaks seen in the conditional mass functions. This trend is not surprising in the context of the central galaxy mass to halo mass relationship shown in Figure 5(b), which follows a monotonic trend. The large drop-off in numbers of centrals in cluster environments (red, ECO only) underscores the rarity of such large objects.

Next we examine the difference between the central galaxy SMF and BMF. It is apparent that in the low group halo mass regime, there is a significant shift $(\sim 0.5 \mathrm{dex})$ in the location of the peak of the central SMFs and BMFs. We have deliberately set the low group halo mass regime to select groups with central galaxies that are below the gas-richness threshold mass, which typically have as much or more neutral gas mass as their stellar mass (K13). The large shift in the peak of the central galaxy $\mathrm{SMF}$ and $\mathrm{BMF}$ in the low halo mass regime simply underscores the importance of including cold gas mass when considering the masses of low-mass central galaxies.

For larger halo mass regimes, the shift in the location of the peak for the central galaxy SMF and BMF is much smaller. In the intermediate halo mass regime, the central stellar and baryonic mass peaks are $\sim 10^{10.2} M_{\odot}$, although we note that the central BMF hump becomes noticeably narrower, and thus the lower mass centrals within this regime still have a significant amount of gas. In the large group halo mass regime, the central stellar and baryonic mass peaks are located at $\sim 10^{10.7} M_{\odot}$, and in the cluster halo mass regime the central mass peaks are located at $\sim 10^{11.3} M_{\odot}$ (the shutdown galaxy mass scale of K13). The lack of a shift in the peak values of the SMF and $\mathrm{BMF}$ reflects that central galaxies in these larger groups and clusters do not have any significant cold gas mass, which is expected since they are above the bimodality mass and they are in dense environments (see, e.g., Davies \& Lewis 1973; Haynes et al. 1984; M15).

\subsubsection{Satellite Galaxy Mass Functions in Each Group Halo Mass Bin}

While the central mass functions follow a pattern of discrete humps, the satellite mass functions exhibit much more complex structure. In the right panels of Figures 14 and 15, we show these complex satellite galaxy mass functions that define the low-mass slopes of the conditional mass functions. For the lowmass halo regime, we do not show the satellite galaxy mass functions since there are so few satellites in this regime with masses greater than the mass completeness limits. We note that since group finding is not perfect (as discussed in Section 3.5.1), the satellite mass functions are subject to issues of purity and completeness of the group halo catalog. As described previously, however, performing this analysis with alternate linking lengths yields similar results, and we do not think these issues significantly affect our results.

In the intermediate group halo mass regime (green), we see that the observed flat low-mass slope described in Section 5.3 is due to the satellite population. We also observe that the 


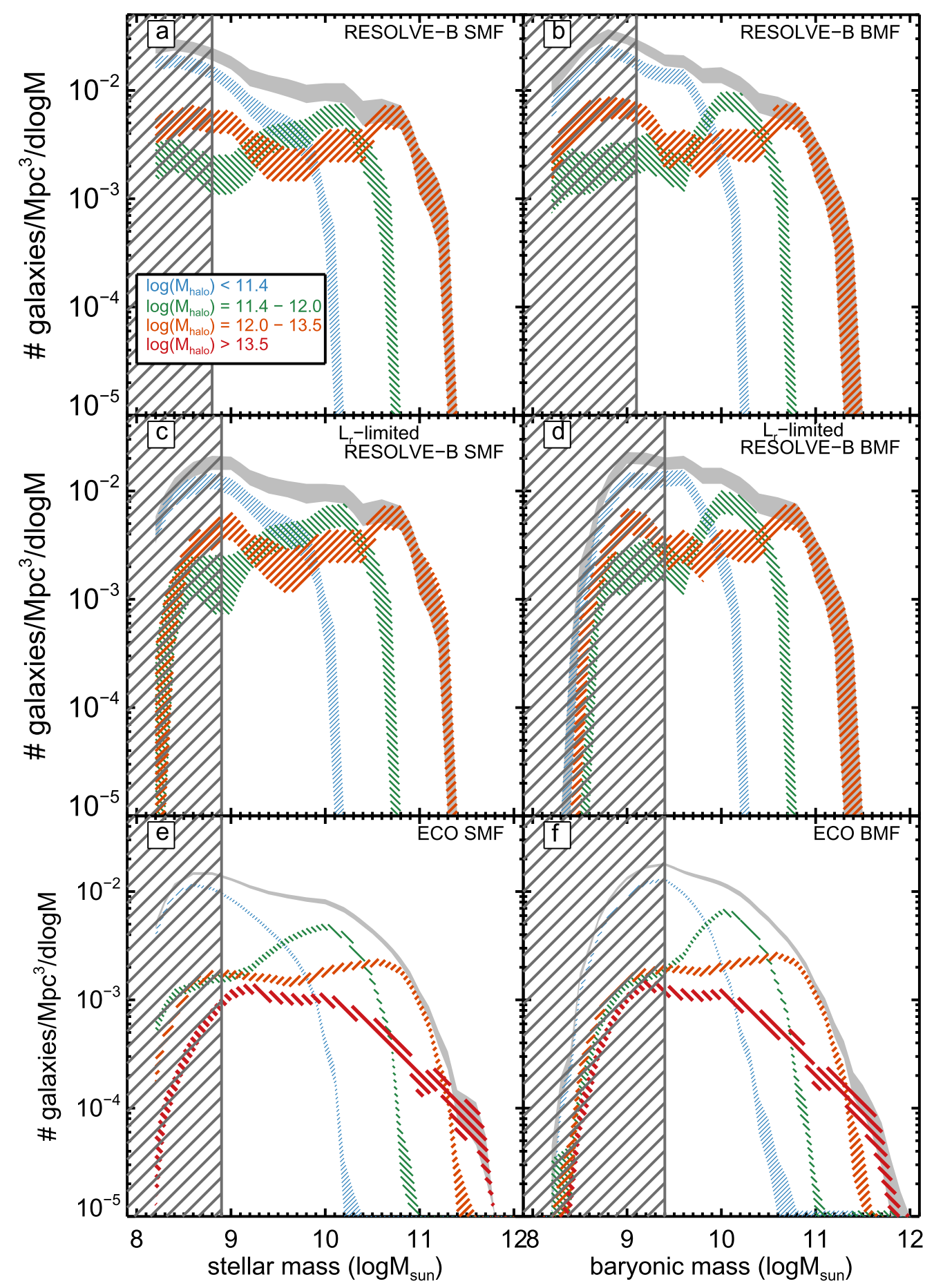

Figure 13. Breakdown of RESOLVE-B (top row), $L_{r \text {,tot }}$-limited RESOLVE-B (middle row), and ECO (bottom row) SMF and BMF into different group halo mass regimes. The four group halo mass regimes are $M_{\text {halo }}<10^{11.4} M_{\odot}$ (low-mass groups, often solo centrals, blue), $M_{\text {halo }}$ between $10^{11.4}$ and $10^{12} M_{\odot}$ (intermediate-mass groups, green), $M_{\text {halo }}$ between $10^{12}$ and $10^{13.5} M_{\odot}$ (large group, orange), and $M_{\text {halo }}>10^{13.5} M_{\odot}$ (cluster, red, ECO only). The dark gray marks the overall SMF or $\mathrm{BMF}$ for each data set, and the gray hash-marked region denotes the incomplete regions for each mass function. The conditional mass functions are much more complex than the overall mass function, with pronounced bumps and dips.

satellites still have a gas component, as the BMF is shifted toward higher mass than the SMF. The shift, however, is not as extreme as for the central galaxy mass function in low group mass halos (blue), which have stellar masses similar to the satellites of the intermediate group halo mass regime but are more gas-rich.

In the large group halo mass regime (orange), the satellite galaxy mass function has a dip (or possibly flat segment) just below $10^{10} M_{\odot}$, even in baryonic mass. For RESOLVE-B, we then find that the satellite galaxy mass function starts to rise again below $10^{9.7} M_{\odot}$, although this rise is not evident for ECO. We also note that for more massive satellite galaxies in large group halos, there is relatively little cold gas as the SMF and BMF are very similar. For galaxies with mass $<10^{10} M_{\odot}$, the BMF is shifted toward slightly higher masses, indicating that some lower mass galaxies do retain a cold gas reservoir. 


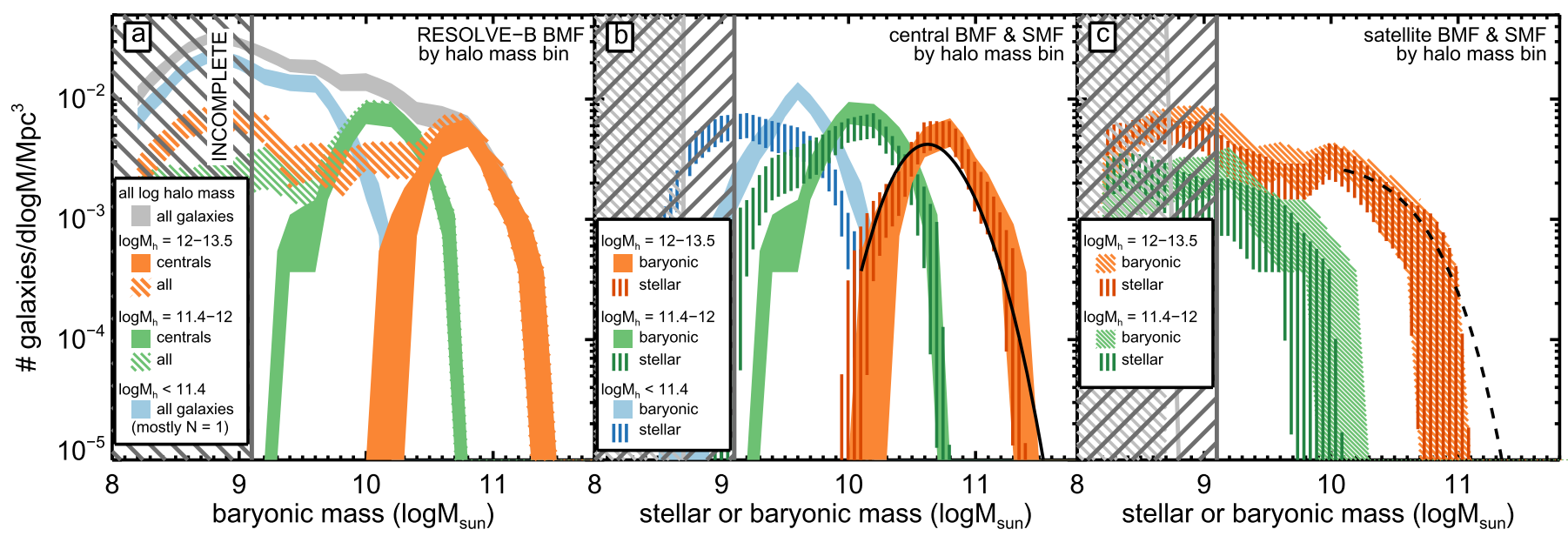

Figure 14. RESOLVE-B SMF and BMF broken down by group halo mass regime and central versus satellite designation. (a) RESOLVE-B BMF with conditional mass functions in crosshatch and central mass functions shown in solid. (b) RESOLVE-B central galaxy SMF (darker crosshatch) and central galaxy BMF (solid). For the central galaxy mass functions, we have applied a halo mass floor in the low-mass halo regime at $M_{\text {halo }}=10^{11.1} M_{\odot}$. (c) RESOLVE-B satellite galaxy SMF (darker crosshatch) and satellite galaxy BMF (lighter crosshatch). The low-mass slope in the intermediate group halo mass regime and the dip in the large group halo mass regime are seen in the satellite population. Incomplete regions are shaded in dark gray for baryonic mass and light gray for stellar mass. In the large group regime, we show the central (solid black line) and satellite (dashed black line) conditional SMFs for mock catalogs from Reddick et al. (2013), which are in rough agreement with our observed mass functions.

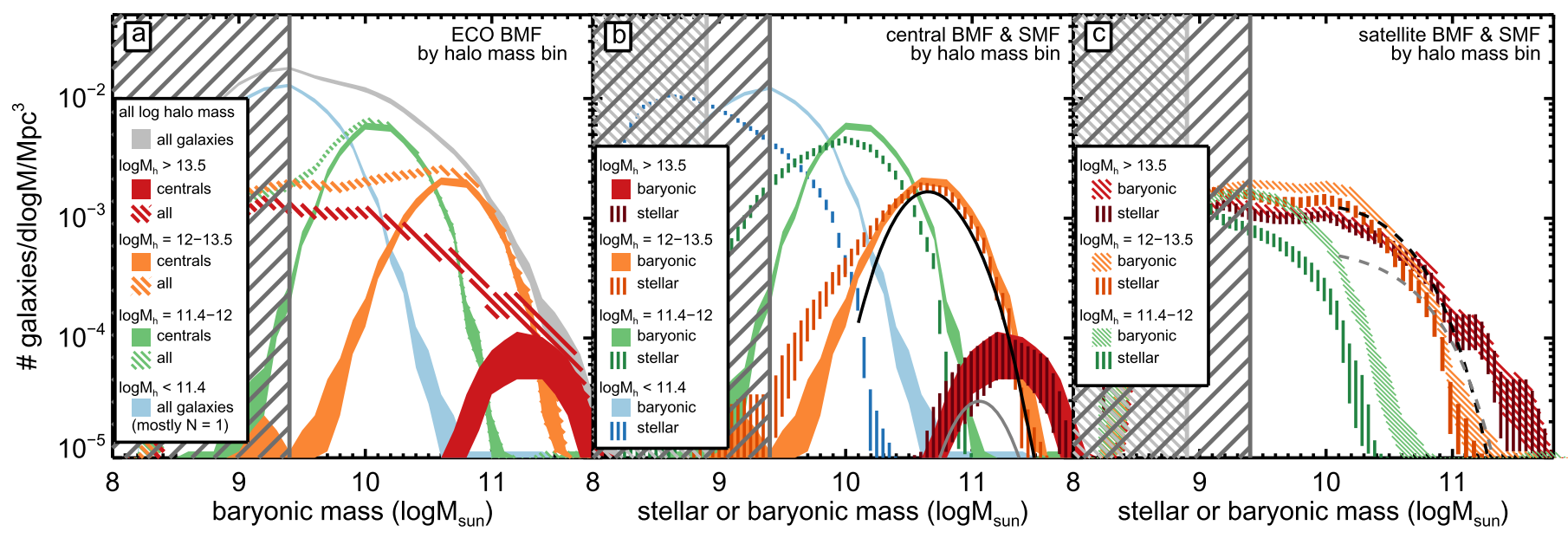

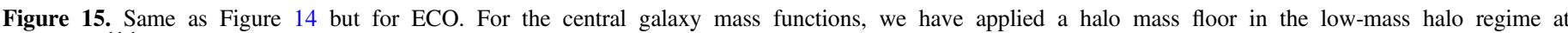

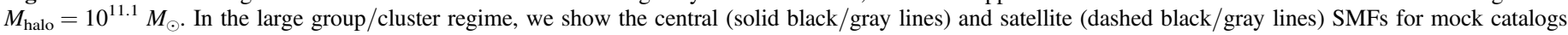

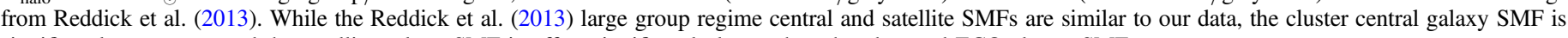
significantly narrower, and the satellite galaxy SMF is offset significantly lower than the observed ECO cluster SMF.

In contrast, the ECO cluster satellite mass function rises more smoothly, although it flattens below galaxy masses of $\sim 10^{10} M_{\odot}$. The SMF and BMF are essentially the same at all masses, indicating that the satellites of such large clusters have very little to no cold gas. Although the satellite galaxy mass function in the cluster appears to have a smoother shape than in the lower halo mass regimes, the cluster should be made up of what were originally smaller groups that have fallen into the larger potential well over time. Thus, all the more intricate shapes of the smaller group halos have combined to form the cluster regime's smoother shape.

The substructure seen in these satellite galaxy mass functions, particularly for intermediate and large group halo masses, may arise from the formation of groups. While there is a 0.3-0.4 shift between the SMF and BMF of galaxies in the low group halo mass regime, indicating large amounts of cold gas in such galaxies, we find a much smaller shift between the satellite SMF and BMF at similar galaxy stellar masses in the intermediate and large group halo mass regimes. This result suggests that gas-removal processes such as ram-pressure or viscous stripping (Gunn \& Gott 1972; Nulsen 1982) or starvation or strangulation (Larson et al. 1980) may already begin even in the intermediate group halo mass regime. This lends support to preprocessing, the idea that galaxies begin to be quenched in smaller groups before falling into the cluster (Zabludoff \& Mulchaey 1998). Perhaps even more intriguing are the dips and varying low-mass slopes seen in the satellite galaxy mass functions. Conditions within early group formation, such as low velocity dispersion among group members, may promote merging at preferred mass scales (Pipino et al. 2014), completely removing satellite galaxies from the overall galaxy population. As these groups fall into larger clusters, where the velocity dispersion increases, the satellite galaxies are less likely to merge, resulting in the smoother appearance of the cluster satellite galaxy mass function. 


\subsubsection{Comparison to Previous Work}

Previous studies of conditional mass functions in both data and models have found similar results, fitting the central contribution of the conditional mass function as a log-normal distribution and the satellite mass function as a Schechter or truncated Schechter function (Zheng et al. 2005; Yang et al. 2009; Moster et al. 2010; Reddick et al. 2013). These works, however, cover only the higher halo mass regimes $>10^{12}$ $M_{\odot}$. In the comparisons below, we have corrected all other conditional mass functions to be in units of $H_{0}=70 \mathrm{~km} \mathrm{~s}^{-1}$.

We directly compare with the central SMFs of Reddick et al. (2013), who used the halo abundance matching technique to assign stellar masses to their simulated halos. To compare with the observed large group and cluster mass regimes, we multiply each of their conditional SMFs, which are subdivided more finely than our own, by the appropriate number of halos in either the RESOLVE-B or ECO data set, coadding the resulting functions in each of our halo mass regimes, and then dividing by the volume of the appropriate data set. The results of this process are shown for the large group halo mass (black) and cluster (gray) regimes in Figures 14 and 15. The overplotted central SMFs are in good agreement with our observed central SMFs, although for the cluster regime, the observed SMF has a wider spread and higher peak mass value than that from Reddick et al. (2013). Within Reddick et al. (2013) there is a comparison with the results of Yang et al. (2009), finding that while qualitatively similar, the central SMFs of Yang et al. (2009) are offset toward higher masses, which is most likely due to the difference in stellar mass estimation. While Reddick et al. (2013) use K CORRECT from Blanton \& Roweis (2007), Yang et al. (2009) use the stellar mass prescription from Bell et al. (2003b). Given that we are in good agreement with Reddick et al. (2013) and also the stellar mass discussion in Section 3.2, we expect a similar offset with Yang et al. (2009).

We also compare our mass functions with the theoretical central SMFs of Moster et al. (2010), who used a stellar mass to galaxy halo mass relationship and stellar mass-dependent clustering of galaxies to assign stellar masses to halos in simulations, constraining the parameters of these functions by comparing their assigned SMF with the SMF from Panter et al. (2007). The central galaxy SMFs in Moster et al. (2010) are binned more finely than in this work, so we examine the range of peak masses for each halo mass regime. The Moster et al. (2010) intermediate halo mass regime has a stellar mass peak ranging between $\sim 10^{9.6} M_{\odot}$ and $10^{10.6} M_{\odot}$, encompassing our central mass peak of $\sim 10^{10.2} M_{\odot}$. In the large group and cluster mass regimes, Moster et al. (2010) measure stellar mass peak ranges that are offset toward slightly larger stellar masses than we observe: a range from $10^{10.6}$ to $10^{11.3} M_{\odot}$ for the large groups and a peak of $10^{11.5} M_{\odot}$ for clusters, while we observe a peak at $10^{10.7} M_{\odot}$ for large groups and $10^{11.3} M_{\odot}$ for clusters.

We can also compare the RESOLVE-B and ECO central BMFs with the theoretical central BMFs predicted in Zheng et al. (2005), which used a smoothed particle hydrodynamics simulation together with the GALFORM semi-analytic model (SAM) of galaxy formation (Cole et al. 2000) to measure the conditional BMF (here, baryonic mass includes both the cold atomic and molecular gas components in addition to the stars). Comparing the central mass peaks from the Zheng et al. (2005) $\mathrm{BMFs}$, we find a range from $10^{10.5}$ to $10^{11.3} M_{\odot}$ for large groups and a range from $10^{11.4}$ to $10^{11.6} M_{\odot}$ for clusters. While the baryonic central mass peak that we measure for large group mass halos $\sim 10^{10.7} M_{\odot}$ is within the range from Zheng et al. (2005), in the cluster regime, their peak masses are slightly $(\sim 0.2$ dex $)$ higher than ours $\left(\sim 10^{11.3} M_{\odot}\right)$ for clusters. Similar discrepancies have been noted in the analysis of Liu et al. (2010), which compares the galaxy stellar mass to halo mass relationship predicted by SAMs with observations, but as we have few massive clusters, we cannot rule out cosmic variance (see Section 5.2.1).

For the satellite mass functions, we can directly compare with those from Reddick et al. (2013). Our satellite mass functions typically drop off in numbers around the center of the peak value of the central galaxy mass function, except in the cluster regime, where the most massive satellites start to outnumber the few centrals associated with these rare halos. In Figures 14 and 15 we show the Reddick et al. (2013) satellite galaxy mass functions in the large group halo mass regime as a black dashed line and in the cluster regime as a gray dashed line. The large group halo mass satellite mass functions generally agree with the results in RESOLVE-B and ECO, although this work could not probe the interesting substructure that occurs below a galaxy mass of $\sim 10^{10} M_{\odot}$. In the cluster regime, the satellite mass functions do not agree with our results, which may be related to the discrepancy in the central mass function.

Overall, we find qualitative agreement with previous work in examining the central and satellite mass functions in different bins. Differences in stellar mass estimation and prescriptions for populating mock catalogs may yield offsets in the peak mass values, but the general pattern for the central conditional mass functions is evident. With RESOLVE-B we are able to extend our analysis to lower halo masses than most previous work, finding intriguing patterns in the intermediate and low group halo mass regimes.

\subsubsection{Mass Function Reconstruction}

Returning to the problem of the high-mass discrepancy between the RESOLVE-B and ECO mass functions, we can now examine whether the overall mass function of a data set simply reflects the survey's group halo mass distribution. We have seen that it is possible to scale the mock catalog conditional mass functions from Reddick et al. (2013) by the appropriate number of halos to match the observed conditional mass functions we observe (Section 5.3.5). If the particular halo mass regime sets the shape of the mass function, then given a basis set of "per group halo" mass functions and a group halo mass distribution for a survey, we should be able to reconstruct the observed galaxy mass function of the survey.

To determine whether such a reconstruction would reconcile RESOLVE-B and ECO, we first derive a basis set of BMFs from ECO. We start with the completeness-corrected BMFs from the four halo mass regimes defined in the previous section, then normalize these four conditional mass functions by the number of group halos in each halo mass regime. Since the ECO mass functions have been completeness corrected, we use the weighted total of centrals in each halo mass regime as the total number of halos in each halo mass regime. We next determine the number of group halos in each group halo mass regime for the data set that we want to reconstruct: RESOLVE$\mathrm{B}$ in this case. For this analysis, we limit RESOLVE-B to galaxies brighter than -17.33 in order to match the selection for ECO. Since RESOLVE-B has no halos with mass $>10^{13.5} M_{\odot}$, we consider only the low, intermediate, and large 
group halo mass regimes from ECO, then multiply the ECO basis functions by the number of RESOLVE-B halos in each group halo mass regime.

The resulting RESOLVE-B and scaled ECO basis BMFs for each halo mass regime are shown in panels (a)-(c) of Figure 16, where the RESOLVE-B conditional mass functions are shown in green and the scaled ECO basis mass functions are shown in blue. The raw ECO mass functions are shown in pink for comparison. To create the total BMF, we simply add the mass functions in each halo mass regime to compare with the actual data. We find good agreement between the observed and reconstructed RESOLVE-B BMFs, including the bump near $\sim 10^{11} M_{\odot}$ and the overall normalization.

The ability to reconstruct the RESOLVE-B BMF from the ECO basis BMFs suggests that the shape of the mass function for a given survey is dependent on the particular group halo mass distribution sampled. This conclusion is not to say that large-scale structure does not play a role, since the group halo mass distribution depends on the large-scale structure. We infer that the group halo mass distribution contributes to differences between the mass functions observed in different studies, particularly for small surveys. For larger data sets where the halo distribution closely resembles the true halo mass distribution, the mass functions should all tend toward the same shape. For smaller data sets, like RESOLVE-B, we expect to find interesting differences in the mass function, such as the prominent high-mass bump in Figure 16(c). This result also supports the claim in Faltenbacher (2010) that the highmass bump seen in other galaxy mass functions is primarily due to the central galaxy populations, and in particular centrals from the large group halo mass regime. The apparent contribution of a bump from red galaxies (Bolzonella et al. 2010), then, is due to the fact that centrals in high-mass halos are more likely to be red.

\section{DISCUSSION}

In this section, we discuss implications of our results for the physics of group formation. We also revisit the discrepancy in low-mass slope between the observed galaxy and theoretical HMFs in light of our analysis.

\subsection{How Group Halo Mass Environment Shapes the Galaxy Mass Function}

In Section 5.3, we showed that underneath the relatively simple shape of the overall galaxy mass function, the conditional galaxy mass functions have much more complex structure. These include regular humps from central galaxies and both dips and varying low-mass slopes from satellite galaxies (e.g., a flat slope in intermediate-mass halos and a steeply rising slope in large group halos). Furthermore, we showed that differences in the overall mass function of different surveys, such as the prominent bump around $\sim 10^{11} M_{\odot}$ in RESOLVE-B, can be explained by scaling a basis set of conditional mass functions by the appropriate group halo mass distribution (see Section 5.3.6 and Figure 16). Together, these observations suggest that groups have a profound effect on shaping the galaxy population. In particular, group formation processes, such as merging and stripping, appear to occur from the onset of nascent group formation in intermediate-mass halos and create the complex structure seen in the conditional mass functions.
In the intermediate and large group halo mass regimes, groups still have relatively few members. In the low group halo mass regime, with $M_{\text {halo }}<10^{11.4} M_{\odot},>90 \%$ of our groups are $N=1$ galaxy systems consisting only of a central galaxy without any satellites brighter than the luminosity completeness limit used for group finding. Transitioning into the intermediate and large group halo mass regimes $\left(M_{\text {halo }}\right.$ between $10^{11.4} M_{\odot}$ and $\left.10^{13.5} M_{\odot}\right)$, we find a population of small groups consisting of two to seven members. In the cluster regime $\left(M_{\text {halo }}>\right.$ $\left.10^{13.5} M_{\odot}\right)$, groups consist of tens to hundreds of members.

Intermediate-mass groups are nascent groups, the site of galaxies first coming together before entering larger groups and clusters. The flat low-mass slope seen in this first group halo mass regime suggests that, within nascent groups, satellite stripping and destruction are already occurring and shaping the galaxy mass functions. Mergers and stripping remove galaxies from the galaxy population or shift them to lower masses, depressing the mass function in the low-mass range. Only relatively rare major mergers substantially increase galaxy masses, while minor mergers repeatedly eliminate counts from the low-mass end of the mass function. Nascent groups may be preferred environments for merging, as the relative speeds of the galaxies in these groups are much smaller than in larger groups and clusters (as found in Pipino et al. 2014). These first groups (and more solitary field galaxies) eventually combine into larger groups, so clusters are essentially made up of a range of smaller group masses. Thus, the cluster mass function represents a linear combination of all of the smaller group mass functions, potentially smoothing the dips and varying low-mass slopes into one broadly rising composite that hides the complexity of its past. This scenario is supported by prior work suggesting the existence of preprocessing, in which galaxies begin quenching in smaller groups before falling into larger clusters (e.g., Zabludoff \& Mulchaey 1998; McGee et al. 2009; Wetzel et al. 2013; Haines et al. 2015). In follow-up work, we will show that the group integrated baryonic to halo mass ratio is relatively flat over this intermediate halo mass range, as these groups are efficiently converting gas to stars (K. D. Eckert et al. 2016, in preparation).

\subsection{Comparison between Galaxy and HMFs}

We now revisit the discrepancy between the low-mass slopes of the overall galaxy SMF and BMF and the theoretical HMF, focusing on the combined subhalo and group HMF, which should map to satellites and centrals. Based on the studies of the baryonic Tully-Fisher relation, which reveal a tight correlation between galaxy cold baryonic mass and rotation velocity even for low-mass galaxies (e.g., McGaugh et al. 2000), we might expect that the BMF should trace the theoretical HMF scaled by the universal baryon fraction. Examining Figure 12, we do in fact find that the low-mass slope of the BMF is steeper than that of the SMF over the mass range below the knee of the mass functions $\left(\alpha_{\mathrm{BMF}} \sim-1.3\right.$ versus $\alpha_{\mathrm{SMF}-\text { plateau }} \sim-1.2$ ). However, the $\mathrm{BMF}$ is still significantly shallower than the universal baryon fraction scaled galaxy HMF including subhalos (see Section 5.2.5 and Figure 12), which has a low-mass slope of $\alpha_{\mathrm{HMF}} \sim-1.84$ (Shankar et al. 2006).

To alleviate this tension, we consider first that the baryonic rotation velocity $V_{\text {disk }}$ may not be directly related to halo circular velocity $V_{\text {halo }}$, since the halo extends much farther than the optical galaxy. Using a combination of lensing to determine 


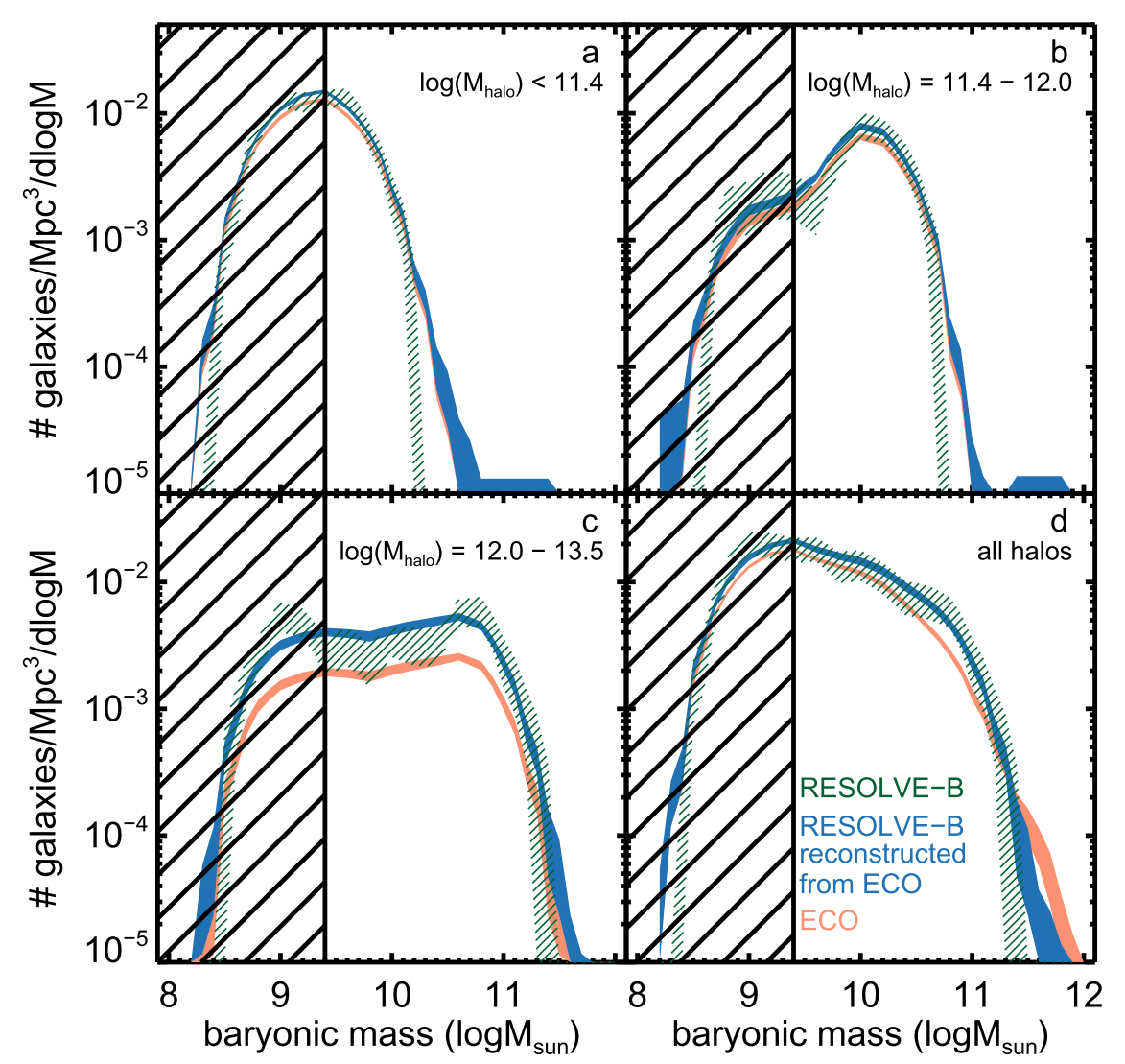

Figure 16. Reconstruction of the RESOLVE-B BMF using the ECO conditional mass functions. Panels (a)-(c) show the conditional BMFs in progressively higher mass halo regimes. The RESOLVE-B BMFs are constructed from an $L_{r \text {,tot }}$-limited data set $\left(M_{r \text {,tot }}<-17.33\right)$ to be consistent with ECO and are shown in crosshatched green. The original ECO BMFs are shown in solid light pink. The reconstructed RESOLVE-B BMFs based on scaling the ECO BMF catalog are shown in cross-hatched blue. The difference in abundance of large group halos in RESOLVE-B and ECO is very apparent in panel (c), but the overall shape of the large group mass functions is similar. In panel (d), we add the three conditional BMFs based on ECO (blue) to compare with the overall RESOLVE-B BMF (green). The reconstructed BMF matches that of RESOLVE-B very well. The observed ECO BMF is offset lower near the large group bump in RESOLVE-B and extends to higher masses since it includes the cluster regime not represented in RESOLVE-B.

halo velocities and Tully-Fisher-derived disk velocities, Reyes et al. (2012) find that for galaxies ranging in mass from $10^{9} M_{\odot}$ to $10^{11} M_{\odot}$ the ratio of $V_{\text {disk }}$ to $V_{\text {halo }}$ ranges from 1.27 to 1.39 . An abundance-matching analysis, however, finds the ratio of $V_{\text {disk }}$ to $V_{\text {halo }}$ to be $\sim 1.5$, but only over a narrow velocity range from 110 to $170 \mathrm{~km} \mathrm{~s}^{-1}$, significantly underestimating $V_{\text {halo }}$ at lower masses (Papastergis et al. 2011). Thus lensing and abundance matching disagree, again returning us to the discrepancy in counts of low-mass galaxies.

Assuming that the $V_{\text {disk }}-V_{\text {halo }}$ correspondence does hold, the next consideration might be that the theoretical HMF coming from $N$-body simulations is incorrect. This second possibility runs counter to the body of evidence supporting the $\Lambda \mathrm{CDM}$ cosmological framework, including measurement of the fluctuations in the cosmic microwave background (Spergel et al. 2003; Planck Collaboration et al. 2014) and successful production of large-scale structure by $\Lambda \mathrm{CDM}$ dark matter simulations (Springel et al. 2005). Despite these successes, however, the low-mass slope mismatch and other discrepancies between observations and simulations (e.g., the core versus cusp problem reviewed in de Blok 2010) have prompted widespread investigation of different forms of dark matter, e.g., warm dark matter, scalar field dark matter, and so on (Magaña \& Matos 2012; Lovell et al. 2014; Velten et al. 2014).

An alternative to considering other forms of dark matter may be that the HMF from $N$-body simulations is incorrect because the simulations lack baryonic physics. Including the baryonic physics of feedback may redistribute dark matter within halos, flattening the inner cuspy profiles of dark matter halos. Very high resolution simulations of low-mass galaxies reveal that even the smallest dwarf galaxies can transform their dark matter cusps to cores as long as star formation can proceed (Read et al. 2016). The authors argue that these dark matter halo transformations may allow for halos to be tidally destroyed more easily. Interpreting this idea in the context of galaxies being destroyed in nascent groups (Section 6.1), we might consider that current $\mathrm{N}$-body simulations without baryonic physics are not destroying enough subhalos, leading to an artificially steep low-mass slope and also throwing off abundance matching. However, this answer may be only partial because Papastergis et al. (2015) argue that there is a missing dwarf problem in the field, outside the group environment.

A third consideration thus arises that cold atomic gas mass may significantly underestimate the collapsed gas mass in dwarfs. Low-mass galaxies often show notably smaller baryon fractions than expected, even when including atomic gas (McGaugh et al. 2010; Papastergis et al. 2012). These extremely low baryon fractions imply deviations from the baryonic Tully-Fisher relation, and indeed some studies find that multiplying the neutral gas content by a factor of 3-11 can tighten the baryonic Tully-Fisher relation further (Pfenniger \& Revaz 2005; Begum et al. 2008; Revaz et al. 2009). These 
results point toward the potential existence of large undetected gas reservoirs of either ionized or ultracold molecular gas in low-mass galaxies. If our observed baryonic masses significantly underestimate true baryonic masses, the true BMF might be steeper and more like the theoretical HMF. We plan to examine this possibility in more detail in a follow-up paper.

For completeness, we note the orthogonal possibility that gas loss driven by feedback explains the slope discrepancy as lower-mass galaxies are less efficient at forming stars. This scenario relies on supernova feedback expelling gas from lowmass halos (Dekel \& Silk 1986), but, based on realistic feedback energies, even dwarf galaxies with masses as low as $10^{7} M_{\odot}$ are able to retain their gas (Mac Low \& Ferrara 1999). A more likely candidate to suppress star formation and reduce cold gas in low-mass galaxies is the strong ionizing UV background from the epoch of reionization, leaving only earlyforming halos able to form stars (Bullock et al. 2000). In this context, Bell et al. (2003a) conjecture a later recooling that could produce an environment-independent BMF, but our conditional mass function results argue against this picture.

One final consideration is the issue of flyby interactions or ejected satellite galaxies (Sinha \& Holley-Bockelmann 2012), which have interacted with a group or cluster but are no longer within that group or cluster's halo. In Wetzel et al. (2014), the authors find that the stellar-to-halo mass relationship for flybys or ejected satellites is much higher than usual, as the halo is stripped during its encounter with the cluster halo. This halo stripping could increase the number of low-mass dark matter halos (in simulations as well as in the real universe) relative to low-mass galaxies, further increasing the mismatch in low-mass slope.

It is possible that there is an element of all of these considerations at play in explaining the differences between observed galaxy and theoretical HMFs. In this work, we have stressed the role of nascent groups in shaping the galaxy population through satellite stripping or merging. In future work, we will more closely examine the possibility that unobservable gas can help to reconcile the galaxy and groupintegrated BMFs with theoretical HMFs.

\section{CONCLUSIONS}

We have constructed two volume-limited data sets, RESOLVE-B and ECO, to study the galaxy SMF and BMF. The RESOLVE-B data set has unprecedented completeness (see Figures 1(b) and 6), which allows us to study the entire galaxy population and produce empirical completeness corrections for the ECO catalog. Using volume-limited surveys allows us to study mass functions without the statistical completeness corrections necessary for magnitude-limited surveys and to define groups of galaxies and assign group halo masses via FOF group finding and halo abundance matching. In Section 4 we present a novel cross-bin sampling method for constructing galaxy mass functions using the full stellar and gas mass likelihood distributions. Our findings are as follows:

1. The SMF and BMF start to diverge for masses $<10^{10.5} M_{\odot}$ and become significantly different below $\sim 10^{9.9} M_{\odot}$ (near the gas-richness threshold scale of K13). The BMF rises as a straight power law, following the traditional Schechter function form, while the SMF plateaus before appearing to rise more steeply below $\sim 10^{9.5} M_{\odot}($ see Section 5.2.4 and Figure 12).
2. While steeper than the SMF's low-mass slope, the BMF's low-mass slope is still much shallower $\left(\alpha_{\mathrm{BMF}} \sim-1.3\right)$ than the predicted slope from theoretical HMFs, alleviating some tension but not fully explaining the discrepancy (Figure 12).

3. The conditional SMF and BMF broken down into four halo mass regimes have more complex structure than the overall galaxy mass functions and reveal that the majority of low-mass galaxies are centrals in low-mass halos without satellites above our survey limits (see Section 5.3 and Figure 13).

4. The conditional mass functions for central galaxies are divided into narrow humps at discrete mass intervals, as expected from the monotonic relationship between galaxy mass and halo mass (see Section 5.3 and Figures 14 and 15).

5. The low-mass slopes of the conditional satellite mass functions vary significantly in different halo mass regimes. In larger group halos, the low-mass slope in RESOLVE-B rises quite steeply below an initial dip, but the same feature is not clear at ECO's shallower depth. In the intermediate group halo masses of nascent multiplegalaxy groups, we find a flat low-mass slope (Figures 13 and 14).

6. These features seen in galaxy mass functions of intermediate and large group halos suggest the possibility that, even in nascent groups, satellite merging or stripping is already shaping the galaxy population. Recent work suggesting that the theoretical HMF may be much shallower in hydrodynamic simulations that include baryonic physics driving dark matter core formation and facilitating tidal stripping (Read et al. 2016) raises the possibility that group formation and satellite destruction may help to explain the discrepancy between the observed galaxy BMF and the theoretical HMF (see Section 6).

7. As evidence of the primacy of group-scale physics in determining the galaxy mass function, we show that scaling a basis set of conditional mass functions from the ECO data set by the group halo mass distribution in RESOLVE-B recovers the shape of the RESOLVE-B data set, including a bump that is due to centrals in large groups (Figure 16).

In future work, we plan to compare the RESOLVE-B and ECO BMFs with semi-analytic models and hydrodynamic simulations to explore the role of group formation in shaping galaxy mass functions. We will also study the group-integrated SMF and BMF to examine the role of unobserved gas in reconciling observed and theoretical mass functions.

We acknowledge the anonymous referee, whose comments have improved this work. We would like to thank Tom Loredo and Michael Blanton for productive conversations on the statistical analysis of mass functions. We are grateful for helpful insights and suggestions from Ashley Baker, Jillian Bellovary, Chris Clemens, Gerald Cecil, Art Champagne, Adrienne Erickcek, and Andrew Baker. We also thank Victor Calderon for providing custom mock catalogs for the computation of cosmic variance and group-finding errors, and we thank Jessi Cisewski for helpful discussions regarding cosmic variance. KE, SK, DS, and MN acknowledge support from NSF CAREER grant AST-0955368. KE and DS were also supported by GAANN Fellowships, and KE, DS, and AM were supported by NC Space Grant Fellowships. AM 
acknowledges support from the NASA Harriet Jenkins fellowship. AM, DS, and KE were supported by the University of North Carolina Royster Society of Fellows.

This work is based on observations from the SDSS. Funding for SDSS-III has been provided by the Alfred P. Sloan Foundation, the participating institutions, the National Science Foundation, and the U.S. Department of Energy Office of Science. The SDSS-III website is http://www.sdss3.org/. SDSSIII is managed by the Astrophysical Research Consortium for the participating institutions of the SDSS-III Collaboration, including the University of Arizona, the Brazilian Participation Group, Brookhaven National Laboratory, Carnegie Mellon University, the University of Florida, the French Participation Group, the German Participation Group, Harvard University, the Instituto de Astrofisica de Canarias, the Michigan State/Notre Dame/JINA Participation Group, Johns Hopkins University, Lawrence Berkeley National Laboratory, Max Planck Institute for Astrophysics, Max Planck Institute for Extraterrestrial Physics, New Mexico State University, New York University, Ohio State University, Pennsylvania State University, University of Portsmouth, Princeton University, the Spanish Participation Group, the University of Tokyo, the University of Utah, Vanderbilt University, the University of Virginia, the University of Washington, and Yale University. This work is based on observations made with the NASA Galaxy Evolution Explorer. GALEX is operated for NASA by the California Institute of Technology under NASA contract NAS5-98034. This publication makes use of data products from the Two Micron All Sky Survey, which is a joint project of the University of Massachusetts and the Infrared Processing and Analysis Center, California Institute of Technology, funded by the National Aeronautics and Space Administration and the National Science Foundation. This work is based in part on data obtained as part of the UKIRT Infrared Deep Sky Survey. This work uses data from the Arecibo Observatory. The Arecibo Observatory is operated by SRI International under a cooperative agreement with the National Science Foundation (AST-1100968) and in alliance with Ana G. Mndez-Universidad Metropolitana and the Universities Space Research Association. This work is based on observations made using the Green Bank Telescope. The National Radio Astronomy Observatory is a facility of the National Science Foundation operated under cooperative agreement by Associated Universities, Inc.

\section{REFERENCES}

Aihara, H., Allende Prieto, C., An, D., et al. 2011, ApJS, 193, 29 Baldry, I. K., Balogh, M. L., Bower, R. G., et al. 2006, MNRAS, 373, 469 Baldry, I. K., Driver, S. P., Loveday, J., et al. 2012, MNRAS, 421, 621 Baldry, I. K., Glazebrook, K., \& Driver, S. P. 2008, MNRAS, 388, 945 Becker, R. H., Fan, X., White, R. L., et al. 2001, AJ, 122, 2850

Begum, A., Chengalur, J. N., Karachentsev, I. D., \& Sharina, M. E. 2008, MNRAS, 386, 138

Behroozi, P. S., Conroy, C., \& Wechsler, R. H. 2010, ApJ, 717, 379

Behroozi, P. S., Wechsler, R. H., \& Conroy, C. 2013, ApJ, 770, 57

Bell, E. F., \& de Jong, R. S. 2001, ApJ, 550, 212

Bell, E. F., McIntosh, D. H., Katz, N., \& Weinberg, M. D. 2003a, ApJL, 585, L117

Bell, E. F., McIntosh, D. H., Katz, N., \& Weinberg, M. D. 2003b, ApJS, 149,289

Berlind, A. A., Frieman, J., Weinberg, D. H., et al. 2006, ApJS, 167, 1 Bernardi, M., Meert, A., Sheth, R. K., et al. 2013, MNRAS, 436, 697 Blanton, M. R., Hogg, D. W., Bahcall, N. A., et al. 2003a, ApJ, 592, 819 Blanton, M. R., Kazin, E., Muna, D., Weaver, B. A., \& Price-Whelan, A. 2011, AJ, 142, 31

Blanton, M. R., Lin, H., Lupton, R. H., et al. 2003b, AJ, 125, 2276

Blanton, M. R., Lupton, R. H., Schlegel, D. J., et al. 2005, ApJ, 631, 208
Blanton, M. R., \& Roweis, S. 2007, AJ, 133, 734

Bolzonella, M., Kovač, K., Pozzetti, L., et al. 2010, A\&A, 524, A76

Boselli, A., Cortese, L., Boquien, M., et al. 2014, A\&A, 564, A66

Bruzual, G., \& Charlot, S. 2003, MNRAS, 344, 1000

Bullock, J. S., Kravtsov, A. V., \& Weinberg, D. H. 2000, ApJ, 539, 517

Calzetti, D. 2001, PASP, 113, 1449

Carollo, C. M., Cibinel, A., Lilly, S. J., et al. 2013, ApJ, 776, 71

Casoli, F., Sauty, S., Gerin, M., et al. 1998, A\&A, 331, 451

Catinella, B., Schiminovich, D., Cortese, L., et al. 2013, MNRAS, 436, 34

Chabrier, G. 2003, PASP, 115, 763

Cole, S., Lacey, C. G., Baugh, C. M., \& Frenk, C. S. 2000, MNRAS, 319, 168

Colless, M., Dalton, G., Maddox, S., et al. 2001, MNRAS, 328, 1039

Conroy, C., \& Wechsler, R. H. 2009, ApJ, 696, 620

Cooray, A. 2006, MNRAS, 365, 842

Davies, R. D., \& Lewis, B. M. 1973, MNRAS, 165, 231

de Blok, W. J. G. 2010, AdAst, 2010, 5

Dekel, A., \& Silk, J. 1986, ApJ, 303, 39

Dressler, A. 1978, ApJ, 223, 765

Driver, S. P., Hill, D. T., Kelvin, L. S., et al. 2011, MNRAS, 413, 971

Driver, S. P., \& Robotham, A. S. G. 2010, MNRAS, 407, 2131

Drory, N., Bundy, K., Leauthaud, A., et al. 2009, ApJ, 707, 1595

Duarte, M., \& Mamon, G. A. 2014, MNRAS, 440, 1763

Dunkley, J., Spergel, D. N., Komatsu, E., et al. 2009, ApJ, 701, 1804

Eckert, K. D., Kannappan, S. J., Stark, D. V., et al. 2015, ApJ, 810, 166

Falco, E. E., Kurtz, M. J., Geller, M. J., et al. 1999, PASP, 111, 438

Faltenbacher, A. 2010, MNRAS, 408, 1113

Foreman-Mackey, D., Hogg, D. W., Lang, D., \& Goodman, J. 2013, PASP, 125,306

Gnedin, N. Y., \& Ostriker, J. P. 1997, ApJ, 486, 581

Goto, T., Okamura, S., McKay, T. A., et al. 2002, PASJ, 54, 515

Gunn, J. E., \& Gott, J. R., III 1972, ApJ, 176, 1

Haines, C. P., Pereira, M. J., Smith, G. P., et al. 2015, ApJ, 806, 101

Hambly, N. C., Collins, R. S., Cross, N. J. G., et al. 2008, MNRAS, 384, 637

Haynes, M. P., Giovanelli, R., \& Chincarini, G. L. 1984, ARA\&A, 22, 445

Haynes, M. P., Giovanelli, R., Martin, A. M., et al. 2011, AJ, 142, 170

Huang, S., Haynes, M. P., Giovanelli, R., \& Brinchmann, J. 2012, ApJ, 756,113

Jones, D. H., Read, M. A., Saunders, W., et al. 2009, MNRAS, 399, 683

Kannappan, S. J. 2004, ApJL, 611, L89

Kannappan, S. J., \& Gawiser, E. 2007, ApJL, 657, L5

Kannappan, S. J., Guie, J. M., \& Baker, A. J. 2009, AJ, 138, 579

Kannappan, S. J., Stark, D. V., Eckert, K. D., et al. 2013, ApJ, 777, 42

Kannappan, S. J., \& Wei, L. H. 2008, in AIP Conf. Ser. 1035, The Evolution of Galaxies Through the Neutral Hydrogen Window, ed. R. Minchin, \& E. Momjian (Melville, NY: AIP), 163

Kauffmann, G., Heckman, T. M., White, S. D. M., et al. 2003a, MNRAS, 341,33

Kauffmann, G., Heckman, T. M., White, S. D. M., et al. 2003b, MNRAS, 341,54

Kim, S., Staveley-Smith, L., Dopita, M. A., et al. 1998, ApJ, 503, 674

Kravtsov, A., Vikhlinin, A., \& Meshscheryakov, A. 2014, arXiv:1401.7329

Larson, R. B., Tinsley, B. M., \& Caldwell, C. N. 1980, ApJ, 237, 692

Leauthaud, A., Tinker, J., Behroozi, P. S., Busha, M. T., \& Wechsler, R. H. 2011, ApJ, 738, 45

Leauthaud, A., Tinker, J., Bundy, K., et al. 2012, ApJ, 744, 159

Li, C., \& White, S. D. M. 2009, MNRAS, 398, 2177

Liu, L., Yang, X., Mo, H. J., van den Bosch, F. C., \& Springel, V. 2010, ApJ, 712,734

Lovell, M. R., Frenk, C. S., Eke, V. R., et al. 2014, MNRAS, 439, 300

Mac Low, M.-M., \& Ferrara, A. 1999, ApJ, 513, 142

Magaña, J., \& Matos, T. 2012, JPhCS, 378, 012012

Markwardt, C. B. 2009, in ASP Conf. Ser. 411, Astronomical Data Analysis Software and Systems XVIII, ed. D. A. Bohlender, D. Durand, \& P. Dowler (San Francisco, CA: ASP), 251

McGaugh, S. S., Schombert, J. M., Bothun, G. D., \& de Blok, W. J. G. 2000, ApJL, 533, L99

McGaugh, S. S., Schombert, J. M., de Blok, W. J. G., \& Zagursky, M. J. 2010, ApJL, 708, L14

McGee, S. L., Balogh, M. L., Bower, R. G., Font, A. S., \& McCarthy, I. G. 2009, MNRAS, 400, 937

Mihos, J. C., Durrell, P. R., Ferrarese, L., et al. 2015, ApJL, 809, L21

Moffett, A. J., Kannappan, S. J., Berlind, A. A., et al. 2015, ApJ, 812, 89

Moré, J. 1978, in Numerical Analysis, ed. G. Watson, Vol. 630 (Berlin: Springer)

More, S., van den Bosch, F. C., Cacciato, M., et al. 2009, MNRAS, 392, 801

Morrissey, P., Conrow, T., Barlow, T. A., et al. 2007, ApJS, 173, 682 
Moster, B. P., Somerville, R. S., Maulbetsch, C., et al. 2010, ApJ, 710, 903 Moster, B. P., Somerville, R. S., Newman, J. A., \& Rix, H.-W. 2011, ApJ, 731, 113

Nulsen, P. E. J. 1982, MNRAS, 198, 1007

Panter, B., Jimenez, R., Heavens, A. F., \& Charlot, S. 2007, MNRAS, 378,1550

Papastergis, E., Cattaneo, A., Huang, S., Giovanelli, R., \& Haynes, M. P. 2012, ApJ, 759, 138

Papastergis, E., Giovanelli, R., Haynes, M. P., \& Shankar, F. 2015, A\&A, 574, A113

Papastergis, E., Martin, A. M., Giovanelli, R., \& Haynes, M. P. 2011, ApJ, 739, 38

Paturel, G., Petit, C., Prugniel, P., et al. 2003, A\&A, 412, 45

Peng, Y.-j., Lilly, S. J., Kovač, K., et al. 2010, ApJ, 721, 193

Pfenniger, D., \& Revaz, Y. 2005, A\&A, 431, 511

Pipino, A., Cibinel, A., Tacchella, S., et al. 2014, ApJ, 797, 127

Planck Collaboration, Ade, P. A. R., Aghanim, N., et al. 2014, A\&A, 571, A16

Popesso, P., Biviano, A., Böhringer, H., \& Romaniello, M. 2006, A\&A, 445, 29

Press, W. H., \& Schechter, P. 1974, ApJ, 187, 425

Pritchard, J. R., Loeb, A., \& Wyithe, J. S. B. 2010, MNRAS, 408, 57

Read, J. I., Agertz, O., \& Collins, M. L. M. 2016, MNRAS, 459, 2573

Read, J. I., \& Trentham, N. 2005, RSPTA, 363, 2693

Reddick, R. M., Wechsler, R. H., Tinker, J. L., \& Behroozi, P. S. 2013, ApJ, 771, 30

Revaz, Y., Pfenniger, D., Combes, F., \& Bournaud, F. 2009, A\&A, 501, 171

Reyes, R., Mandelbaum, R., Gunn, J. E., et al. 2012, MNRAS, 425, 2610

Robotham, A., Wallace, C., Phillipps, S., \& De Propris, R. 2006, ApJ, 652, 1077

Robotham, A. S. G., Norberg, P., Driver, S. P., et al. 2011, MNRAS, 416, 2640

Roediger, J. C., \& Courteau, S. 2015, MNRAS, 452, 3209

Salim, S., Charlot, S., Rich, R. M., et al. 2005, ApJL, 619, L39
Salim, S., Rich, R. M., Charlot, S., et al. 2007, ApJS, 173, 267

Shankar, F., Lapi, A., Salucci, P., De Zotti, G., \& Danese, L. 2006, ApJ, 643, 14

Sinha, M., \& Holley-Bockelmann, K. 2012, ApJ, 751, 17

Skrutskie, M. F., Cutri, R. M., Stiening, R., et al. 2006, AJ, 131, 1163

Smith, R. E. 2012, MNRAS, 426, 531

Somerville, R. S. 2002, ApJL, 572, L23

Spergel, D. N., Verde, L., Peiris, H. V., et al. 2003, ApJS, 148, 175

Springel, V., White, S. D. M., Jenkins, A., et al. 2005, Natur, 435, 629

Stark, D. V., Kannappan, S. J., Eckert, K. D., et al. 2016, ApJ, submitted

Stoughton, C., Lupton, R. H., Bernardi, M., et al. 2002, AJ, 123, 485

Strauss, M. A., Weinberg, D. H., Lupton, R. H., et al. 2002, AJ, 124, 1810

Thoul, A. A., \& Weinberg, D. H. 1996, ApJ, 465, 608

Trenti, M., \& Stiavelli, M. 2008, ApJ, 676, 767

Tully, R. B., Somerville, R. S., Trentham, N., \& Verheijen, M. A. W. 2002, ApJ, 569, 573

Valotto, C. A., Nicotra, M. A., Muriel, H., \& Lambas, D. G. 1997, ApJ, 479, 90

van Dokkum, P. G., Abraham, R., Merritt, A., et al. 2015, ApJL, 798, L45

Velten, H., Caramês, T. R. P., Fabris, J. C., Casarini, L., \& Batista, R. C. 2014, PhRvD, 90, 123526

Warren, M. S., Abazajian, K., Holz, D. E., \& Teodoro, L. 2006, ApJ, 646, 881

Wetzel, A. R., Tinker, J. L., Conroy, C., \& van den Bosch, F. C. 2013, MNRAS, 432, 336

Wetzel, A. R., Tinker, J. L., Conroy, C., \& van den Bosch, F. C. 2014, MNRAS, 439, 2687

Yamanoi, H., Komiyama, Y., Yagi, M., et al. 2012, AJ, 144, 40

Yang, X., Mo, H. J., \& van den Bosch, F. C. 2008, ApJ, 676, 248

Yang, X., Mo, H. J., \& van den Bosch, F. C. 2009, ApJ, 695, 900

York, D. G., Adelman, J., Anderson, J. E., Jr., et al. 2000, AJ, 120, 1579

Zabludoff, A. I., \& Mulchaey, J. S. 1998, ApJL, 498, L5

Zheng, Z., Berlind, A. A., Weinberg, D. H., et al. 2005, ApJ, 633, 791 